\title{
Reliability Study of the Sedation-Agitation Scale in an Intensive Care Unit
}

\author{
by
}

Michelle Ryder-Lewis

\begin{abstract}
A thesis submitted to the Victoria University of Wellington
in partial fulfilment of the

requirements for the degree of

Master of Arts (Applied)

in Nursing
\end{abstract}

Victoria University of Wellington 


\section{Abstract}

The management of sedation in critically ill patients is a complex issue for Intensive Care Units (ICU) worldwide. Notable complications of sedation practices have been identified and efforts to modify these practices in ICUs have begun. While sedation-scoring tools have been introduced into clinical practice in intensive care few have been tested for validity and reliability. One tool which has reliability and validity established is the Sedation-Agitation Scale (SAS). This study is an extension of a previous study by Riker, Picard and Fraser (1999) to determine whether doctors and nurses rate patients similarly using the SAS in a natural ICU setting. It is essential to establish whether these different professionals provide consistent scores and have a mutual understanding of the SAS and its constituent levels. This will help ensure that clinical decisions relating to sedation-needs can be made appropriately and consistently. This quasi-experimental reliability study was set in a 12-bed tertiary general ICU in New Zealand. The SAS had recently been introduced into this unit and a convenience sample of 42 nursing and medical staff performed paired ratings on 69 randomly selected adult ICU patients over an eight week time frame. The mean patient age was 58 years, and $79 \%$ of patients were on continuous infusions of Propofol. Intubated patients made up $91 \%$ of the sample. $74 \%$ of patients were given the same SAS score by the doctor-nurse pair. The weighted kappa score for inter-rater agreement was 0.82 indicating very good agreement. Of the $26 \%$ of scores where there was a difference, the two readings were only one score apart. Most of the difference occurred around SAS scores of 1-2 and 3-4. Further analysis found no staff or patient variables to be statistically significant in impacting on the ratings. The SAS was found to be a reliable sedation-scoring tool in a general ICU when used by nurses and doctors of varying experience. The implementation of the SAS should improve the quality of sedation management in critically ill patients, facilitate communication between nurses and medical staff with regard to the effectiveness of sedation regimes, and assist with the development of optimal sedation and analgesia guidelines for ICU patients. 


\section{Acknowledgement}

I wish to thank my supervisor Kathy Nelson, without your guidance, knowledge, encouragement and ability to keep me on track this would not have been possible. I also wish to acknowledge the support of Lou Gallagher, Biostatician from the Victoria University of Wellington Health Services Research Centre, thank you for your advice and guidance with the statistical analysis. I wish to also acknowledge the support of the Graduate School of Nursing and Midwifery at Victoria University for providing me with the opportunity to undertake this journey.

It is important that I acknowledge the support of the team I am part of in the Wellington Intensive Care Unit. To all the staff there, thank you for your encouragement and understanding this year and your enthusiasm in supporting this research. In particular thank you to my colleagues Dr Peter Hicks and Dr Bob Ure who got me hooked on sedation and put up with my incessant questions this year. A special thank you lan Elson and Celia Mckiernan for giving me study time and the space to carry out this project. I hope this will inspire other nurses to undertake post-graduate education and research in the ICU. Nurses can make a difference to patient care in this setting.

A thesis requires an immense commitment not only by me but also from my family and friends. Thank you to you all for putting up with my lack of contact and at times introspective manner. I wish to thank a special friend Denise Blanchard who has continuously inspired my practice and challenged my thinking. Your support has been so much appreciated. A special thank you to my husband Simon who still married me just as I was about to embark on this journey. Your patience and understanding has astounded me, you have been my rock and this thesis has been our team effort.

This thesis is dedicated to the memory of my parents and mother in law, Margaret Ryder-Lewis who taught me that with hard work anyone can achieve their dreams. Margaret in losing you this year meant you didn't get to see the finished product of something you started with me. You taught me to believe in myself. 


\section{Table of Contents}

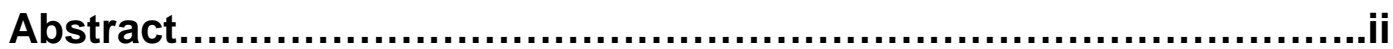

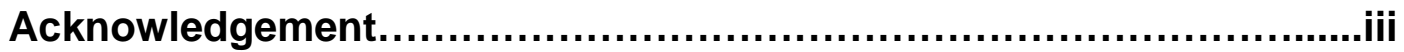

Table of Contents...............................................................

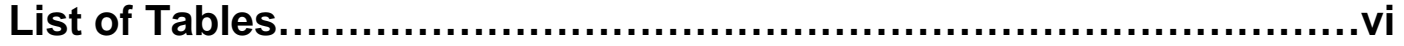

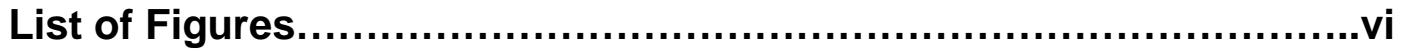

List of Abbreviations........................................................

Chapter 1- Introduction....................................................... 1

Introduction of the Sedation-Agitation Scale to Wellington.................. 4

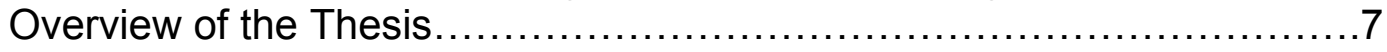

Chapter 2- The Context of Intensive Care................................... 9

The Socio-Historical Context of Intensive Care ............................ 9

Nursing and Technology in Intensive Care................................14

Factors Influencing Clinical Decision-Making in Intensive Care............ 19

The Relationship between Nursing and Medicine in Intensive Care.......26

Nurse-Doctor Interactions in Intensive Care.............................. 31

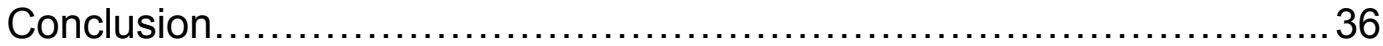

Chapter 3- Sedation in Intensive Care..................................... 39

Aim of Sedation......................................................... 40

Risks and Benefits of Sedation....................................... 42

Sedation Management in Intensive Care .................................43

Common Sedatives Used in the Wellington Intensive Care Unit............45

Nursing Assessment of Sedation....................................... 49

Sedation-Scoring Tools ............................................... 58

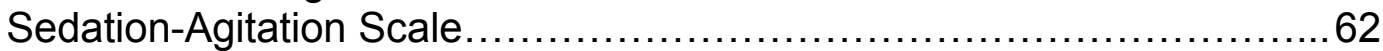

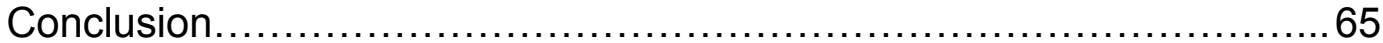

Chapter 4- The Sedation-Agitation Scale................................ 67

The Sedation-Agitation Scale Raters..................................................67

Number of Ratings and Setting ....................................... 70

Selection of People Rated.............................................. 73

Validity of the Sedation-Agitation Scale................................ 73

Reliability of the Sedation-Agitation Scale.............................76

Conclusion...............................................................

Chapter 5- Quasi-Experimental Methodology and Study Design.....79

Quasi-Experimental Research Design.................................. 80

True Score Theory and Measurement Error............................. 83

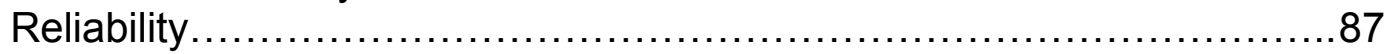

The Relationship between Reliability and Validity Testing................. 95

Study Design and Method.............................................. 96

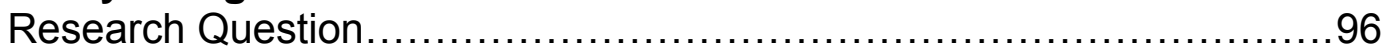

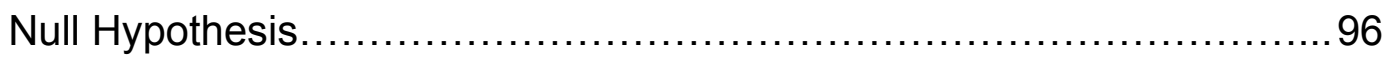




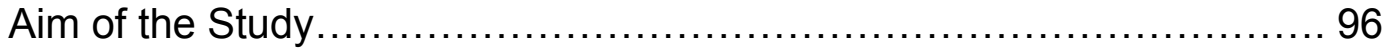

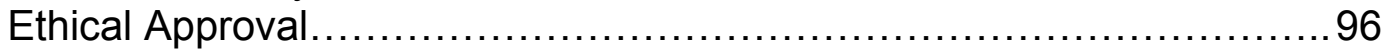

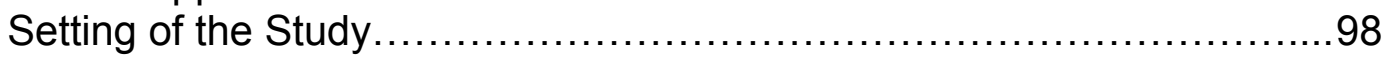

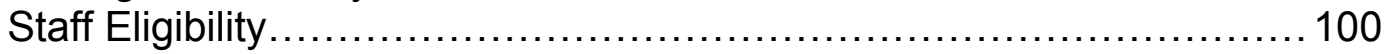

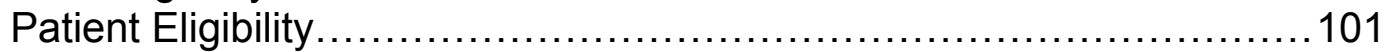

Data Collection Tools.................................................... 102

Method of Data Collection............................................... 104

Data Analysis....................................................... 106

Rigour of the Research............................................ 108

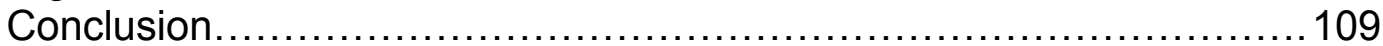

Chapter 6- The Findings............................................ 111

The Staff Sample in the Intensive Care Unit......................... 112

The Patient Population................................................. 114

Clinical Features of Sedation in Wellington............................. 116

Nurse-Doctor Sedation-Scoring in Wellington......................... 117

The Reliability of the Sedation-Agitation Scale in Wellington...............118

The Difference in Agreement........................................ 119

Conclusion...................................................... 121

Chapter 7- Discussion..................................................... 123

The Patient and Staff Sample.................................... 124

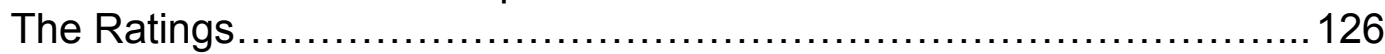

The Sedation-Agitation Scale....................................... 131

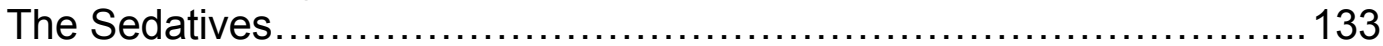

Limitations of the Study and the Sedation-Agitation Scale.............. 134

Reflection on the Research Process.................................. 135

Conclusion..................................................... 137

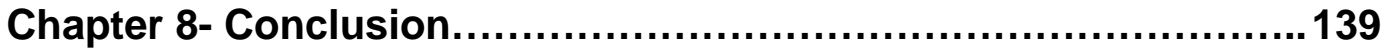

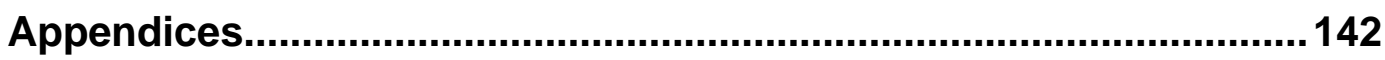

Appendix A- Sedation-Agitation Scale (SAS) .......................... 142

Appendix B- Ethics Committee Approval Letter....................... 143

Appendix C- Family Information Sheets................................ 145

Appendix D- Participant Information and Consent Form...................148

Appendix E- Ethics Committee Safety Concerns........................ 152

Appendix F- Sedation Score Data Sheet............................... 155

Appendix G- APACHE II Diagnostic Codes............................. 156

Appendix H- Letters Confirming Permission to Reprint................... 157

References............................................................. 161 


\section{List of Tables}

Table 1. Subjective Scales to Rate Sedation and Agitation................60 60

Table 2. The Original Sedation-Agitation Scale of $1994 \ldots \ldots \ldots \ldots \ldots \ldots . . . . .63$

Table 3. The Modified Sedation-Agitation Scale of $1999 \ldots \ldots \ldots \ldots \ldots \ldots . . . . . .63$

Table 4. Methodological Evaluation of Sedation-Scoring Tools............ 76

Table 5. Staff Participant Particulars ......................................... 102

Table 6. Data Collected on the Data Collection Tool...................... 102

Table 7. Process for Sedation-Agitation Scale Assessment.............. 106

Table 8. Demographic Detail of Staff Participants........................ 112

Table 9. Experience of Staff Participants who Completed Ratings........113

Table10. Demographic Characteristics of Patients Being Assessed using the Sedation-Agitation Scale................................... 115

Table11. Clinical Features of Patients Being Assessed using the Sedation-Agitation Scale.................................... 116

Table12. Sedatives used by Sample from August-September 2003......117

Table13. Numbers of Agreement by Sedation-Agitation Scale........... 117

Table14. Difference between Nurses and Doctors Ratings............... 119

Table15. The Direction of Difference between the Nurses and Doctors using the Sedation-Agitation Scale............................120

Table16. Patient Variables Impact on Ratings......................... 121

\section{List of Figures}

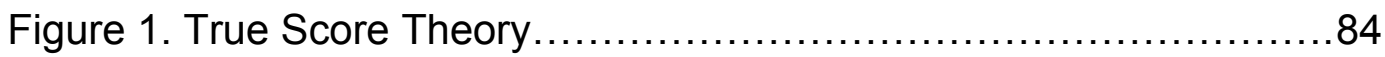

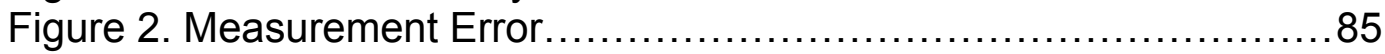

Figure 3. Random Error .............................................. 86

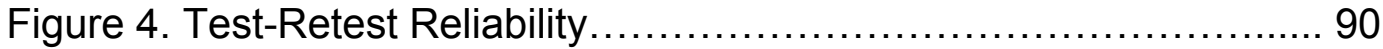

Figure 5. Inter-rater Reliability Test.................................... 91

Figure 6. Parallel Reliability Test....................................... 94

Figure 7. Interpretation of Kappa.................................... 108

Figure 8. Nurses and Doctors Sedation-Agitation Scale Ratings.........118 


\section{List of Abbreviations}

$\begin{array}{ll}\text { APACHE } & \text { Acute Physiology and Chronic Health Evaluation } \\ \text { ANZICS } & \text { Australia and New Zealand Intensive Care Society } \\ \text { CCP } & \text { Clinical Career Pathway } \\ \text { C\&CDHB } & \text { Capital and Coast District Health Board } \\ \text { CO } & \text { Cardiac Output } \\ \text { CNC } & \text { Clinical Nurse Co-ordinator } \\ \text { CNP } & \text { Clinical Nurse Preceptor } \\ \text { CNS } & \text { Clinical Nurse Specialist } \\ \text { CT } & \text { Computed Tomography } \\ \text { Dr. } & \text { Doctor } \\ \text { EAP } & \text { Employee Assistance Programme } \\ \text { EEG } & \text { Electroencephalography } \\ \text { ET } & \text { Endotracheal } \\ \text { GCS } & \text { Glasgow Coma Scale } \\ \text { ICU } & \text { Intensive Care Unit } \\ \text { IPPV } & \text { Intermittent Positive Pressure Ventilation } \\ \text { IV } & \text { Intravenously } \\ \text { K } & \text { Weighted Kappa Statistic } \\ \text { LOS } & \text { Length of Stay } \\ \text { MAAS } & \text { Motor Activity Assessment Scale } \\ \text { MAP } & \text { Mean Arterial Pressure } \\ \text { MOH } & \text { Ministry of Health } \\ \text { No. } & \text { Number } \\ \text { PICU } & \text { Paediatric Intensive Care Unit } \\ \text { SAS } & \text { Sedation-Agitation Scale } \\ \text { Sd } & \text { Standard Deviation } \\ \text { SPSS } & \text { Statistical Package for the Social Sciences } \\ \text { SVR } & \text { Systemic Vascular Resistance } \\ \text { UK } & \text { United Kingdom } \\ \text { USA } & \text { United States of America } \\ \text { VAS } & \text { Visual Analogue Scale } \\ & \end{array}$




\section{Chapter 1- Introduction}

The word 'sedate' is derived from the Latin word sedare, which means 'to calm'. It is a non-specific word used to explain the action of an agent as it moves a patient toward a calm relaxed state. It is estimated that $90 \%$ of critically ill patients require sedation and analgesia for at least part of their stay in an Intensive Care Unit (ICU) (Devlin, Fraser, Kanji, \& Riker, 2001). For some patients sedatives are used to provide intermittent respite from mild anxiety whilst for others it is essential in addressing more significant anxiety or agitation and ensuring physiological stability.

This thesis is reporting on a study looking at the reliability of the SedationAgitation Scale (SAS) which has been introduced into the ICU at Wellington Hospital. This tool is used to assess patients' behaviour and quantify the degree of sedation and agitation which would enable the sedation goal for individual patients' to be determined. This chapter provides an overview of the complexity of the issues surrounding sedation management in ICU. It is acknowledged that the terms ICU and critical care are often used interchangeably in the literature and this is also the case throughout this thesis.

Although non-pharmacological techniques (e.g. correct positioning, minimising noxious environmental stimuli, re-establishing sleep cycles, frequent reorientation, reassurance, and relaxation techniques) are essential, they may not be enough to ensure patient comfort single-handedly. Often sedative medication is also necessary. Sedation can minimise agitation, promote synchronised breathing with the ventilator, and assist to relieve anxiety and discomfort associated with the highly technological environment of the ICU. Sedation is therefore an integral part of the management of many critically ill patients. It is recognised that it is difficult to consider sedation without also considering analgesia because both are frequently required to provide comfort for patients. Sedatives are often used in conjunction with analgesics to manage anxiety and 
agitation beyond the point of pain alone. However, this thesis pertains to sedation assessment only.

Despite the fact that sedatives are used commonly in ICUs internationally, two questions remain; how do we know whether these sedation regimes are effective? And how do we move beyond the simple administration of sedative medication to the regular and consistent evaluation of the efficacy of sedation management? Recently notable complications of sedation practices have been identified and efforts to alter these practices in ICUs have begun. There are substantial international differences in the clinical use of medications for sedative therapy and analgesic control; likewise in the use of sedation scales to assess levels of sedation (Botha \& LeBlanc, 2002; Magarey, 1997; Soliman, Mèlot, \& Vincent, 2001). These practices may also differ within countries depending upon the population of patients and the culture of individual ICUs. The spectrum of differences is an indication of how difficult it is to manage sedation in critically ill patients and how in a time of evidence-based practice a 'gold standard' for sedation management has yet to be identified. There are a vast number of factors which influence how sedation in ICU is managed, both on a micro and macro level. Nurses have a key role to play in the sedation management practices as they are continuously at the bedside of a critically ill patient.

Sedation management has two elements; the assessment and the prescribing/administration of sedation. These are intertwined, nurses and doctors require knowledge of both in order to provide safe and appropriate care to their patients. Increased appreciation of the role of sedation has developed an awareness that subjective assessments of individual patients, even when considering objective components (such as heart rate and respiratory rate), is not enough to consistently determine the effectiveness of sedation regimes. Sedation can be more effectively managed with objective assessments and that has led to the development of sedation-scoring tools. Sedation-scoring tools aim to provide a consistent way of assessing patient behaviour (that may range from deeply 
sedated to wildly agitated) and titrating sedatives accordingly to achieve predefined goals. In many instances it is nursing staff that carry out these assessments and are then required to titrate medications. These tools assist to objectify the subjective component of sedation assessment.

Many sedation-scoring tools, however, have not been established as reliable instruments for use clinically. This does not mean that they are unreliable, only that formal reliability testing has not been carried out. Reliability testing is important to ascertain the ability of an instrument to get the same results by different observers, on different occasions, and/or by similar or parallel tests that produce identical or similar results. Otherwise the instrument's clinical application will be negated. One tool which has reliability established is the SedationAgitation Scale (SAS) (Riker, Picard, \& Fraser, 1999) (see Appendix A). This tool has been shown to be reliable in certain ICU contexts. The SAS uses a 7-point scale to assess patient behaviours that indicates the spectrum of sedation from whether a patient is unrousable to dangerously agitated. It is used to determine the need for sedatives. The SAS is commonly used by nurses as they are continuously monitoring critically ill patients and therefore are more likely to become aware of subtle changes in patients' behaviour. However nurses and doctors require a common understanding of how the SAS works and what the various levels mean to ensure that clinical decisions based on a SAS score can be made appropriately and consistently. What is not known is whether the SAS is reliable in ICUs outside of the United States of America (USA) or whether nurses and doctors using the SAS rate patients similarly. These points and a detailed description of the SAS are discussed further in chapters three and four. The research question for this study consequently is; in the real world, with minimum exclusion criteria and a randomly selected ICU population do nurses and doctors in a tertiary level ICU rate patients' using the SAS similarly? The aim of this thesis was to determine whether the SAS when utilised in a tertiary level general ICU in New Zealand was reliable and whether nurses and doctors had congruence in their ratings. If nurses and doctors do not have a shared appreciation of the terms used to describe sedation, a patients' behaviour or a 
means of determining a patients' level of sedation, then the SAS should not be the basis upon which clinical decisions are made.

This study was undertaken in the general ICU at Wellington Hospital. This ICU had introduced the SAS into clinical practice in February 2003. A three stage process was planned for its implementation. The first stage was training all the staff on the SAS and providing time for them to become familiar with the tool. The second stage was to test the reliability of the SAS which is the aim of the project reported in this thesis. If the SAS is found to be reliable, then the third stage will involve staff using the tool to make clinical decisions regarding the management of patient sedation.

\section{Introduction of the Sedation-Agitation Scale to Wellington}

In 2002, the possible introduction of a sedation-scoring tool to Wellington ICU was first discussed between the senior nursing and medical group. There had been concerns raised that some patients were over-sedated and consequently took some time to wean from mechanical ventilation. After an extensive literature search and an analysis of the literature, a 'short-list' of validated tools that may be appropriate for the ICU were evaluated. This process was guided by a systematic review published by De Jonghe et al. (2000). The choice of sedationscoring tools was then narrowed to two tools; the SAS and the Motor Activity Assessment Scale (MAAS) (Devlin et al., 1999).

Selecting a health assessment tool can be very complicated. Instruments have different advantages and disadvantages. For a tool to be appropriate for clinical practice there are certain issues that need to be considered. Firstly, does the tool require special skills or knowledge to administer? This was important in considering the choices available with sedation-scoring tools as obtaining the skills and knowledge may be costly or require specific training. It became evident early in the evaluation of the literature that many of these tools would require an education program for their use and when examining the literature only a small number of instruments had been published with educational guidelines. 
Secondly, the cost of such an assessment tool needs to be considered. There was a time and resource cost of implementing the SAS in Wellington ICU. As the Clinical Nurse Specialist (CNS) of the ICU, there was my time spent searching and evaluating the literature, and the time of educating over 100 staff in the ICU and the cost of resources such as photocopying, inter-loaning of articles and the development of education resources. An additional cost yet to be addressed, now that the tool has been instituted in the ICU, is the 24-hour flow charts used to record patients' vital signs. If the SAS was found to be a reliable tool for use in the ICU, then these charts would need to be altered to reflect the SAS.

Other considerations in assessing an instrument consist of the coverage of sedation and agitation that each entails. Some sedation-scoring tools do not consider agitation as part of the sedation continuum or it is mentioned only briefly, whilst others focus on compliance with mechanical ventilation. It is essential for individual ICUs to choose a tool that is appropriate for their needs (Robins, 1995). Robins also suggests that if more than one tool is found to be appropriate for an ICU then other issues such as the efficiency of the tool, the clarity and acceptability of the tool and the reliability and validity of the instrument should also be considered. This is why in Wellington ICU the staff were asked to try the SAS and the MAAS in their clinical practice, to help determine which was more acceptable for that particular unit. The information provided by the staff was then evaluated against the reliability and validity of the tools that was presented in De Jonghe et al. (2000). There is no one 'perfect' instrument. The choice made by individual ICUs will depend very much on the culture of the ICU, the goals of the tool and the resources available (Robins).

The SAS and MAAS were trialled in the ICU for a period of two months by a small group of nursing staff. The nurses selected included; permanent night staff, newly appointed staff nurses, experienced nurses and those doing a rotating shift pattern. It was important that the ICU received appropriate feedback from a sample of nurses that represented the range of the nursing population in the unit. The nurses completed an evaluation while using the tools and commented on 
their individual experience with each of the tools. The unanimous decision from the nursing staff was the SAS was preferred over the MAAS. The reasons given by the nursing staff included; its ease of use, simple clear language that the nurses could identify with, and the behavioural descriptors at each level were logical and easy to apply. The nursing staff also reported that they liked the descriptors of agitation over the spectrum of severity that was contained within the tool. Despite the fact that the SAS tool was developed in the USA, the nurses in Wellington ICU found that it suited the culture of their unit and the language used was familiar. The SAS was introduced into practice in February 2003.

Following the theory of Rogers (2003) certain characteristics of innovations as perceived by staff help to explain the rate of adoption. Firstly, the relative advantage is the extent that the innovation is perceived as superior to the current idea or practice. Secondly, the compatibility is the degree to which the SAS may be perceived as being in harmony with existing values, past experiences and the needs of the ICU staff. Thirdly, the complexity of the innovation and whether it is viewed as difficult to understand and use. Fourthly, the trialability is the extent to which the SAS may be experimented with on a limited basis. Finally, the observability, is the degree to which the effect of the SAS innovation is evident to others. In summary innovations that are noted by the recipient as having these components, are likely to be adopted more rapidly than other innovations (Rogers).

All nursing and medical staff in Wellington ICU were educated on how to use the tool, its purpose and the implications of its use in the unit. New staff to the unit are provided with education on the SAS during their orientation phase. A copy of the scale is available at every bed space and assessments are recorded on the patient's 24-hour flow chart. With so many caregivers assessing sedation and agitation in the ICU at various times, it was essential that a tool was chosen that had some established validity and reliability. For a scoring tool to be effective it is important that the staff assessing patients and making clinical decisions, based on those assessments, have an understanding of what is meant by each level of 
the sedation-scoring tool. The SAS was deemed one of the more reliable scoring tools from Wellington ICU's evaluation of the literature. Introducing a sedationscoring tool to the unit focused attention on sedation and agitation as a central component of patient care. Whilst the tool is now being used in the ICU, the intention was that clinical decisions were not to be based on the assessments made from it. This was to allow time for the staff to become familiar with the tool and to allow this research study to take place, to confirm its reliability.

\section{Overview of the Thesis}

This thesis presents the reliability study performed in Wellington ICU. This chapter has introduced the thesis, the background and origin of the research question. It also affords some background to how the SAS was introduced into practice in the Wellington ICU. Chapter Two provides some insight into the sociohistorical context of ICUs and some of the forces impinging on the ICU environment that influence practice. It also provides a justification for the relevance of the research question, namely that nurses and doctors practice differently, have different responsibilities and this may have an impact when they are using the SAS. This chapter also considers the impact of nursing and technology in ICU and the influences on nurses' clinical decision-making which in turn may influence how nurses assess patients using the SAS and manage their sedation needs.

Chapter Three provides further detail to the background discussion of this thesis by identifying the place of sedation in intensive care; its aims, risks and benefits, and what role sedation has in altering the stress response. This is essential as it reveals the complications of sedation practices that can occur. There is also an examination of how nurses assess patients' sedative needs and the complexities of managing sedation in critically ill patients. Finally a discussion of sedationscoring tools is provided and a detailed introduction to the SAS. 
Chapter Four is an analysis of the published literature surrounding the SAS. It focuses on two key studies which have previously tested the inter-rater reliability of the SAS in various settings and with various participants. This chapter identifies the gaps in the literature surrounding the SAS and limitations of the previous research. All of which provide for the origin of this research question and research design.

Chapter Five provides the methodology and study design; the foundation of reliability, measurement error and true score theory. The second section of the chapter conveys how the methodology was applied to the research design. This study is a quasi-experimental design, performed in a natural ICU setting. The aim of the study, null hypothesis and research question are presented along with details of the data collection tool used, the process of the ratings and how the data was analysed.

Chapter Six then presents the findings of the study along with descriptions of the demographics of both the staff and patient participants. Graphs and figures are used to summarise the data. Chapter Seven subsequently takes these findings and reflects on them with a discussion on what the results mean for the research and for clinical practice. Recommendations for further research and clinical practice are presented, along with a consideration of the limitations of this study and the SAS, and a reflection on the research process. Finally the conclusion brings together the key themes that have evolved throughout this thesis and justify the importance of this research study. 


\section{Chapter 2- The Context of Intensive Care}

Before further consideration of sedation management in the Intensive Care Unit (ICU) can be contemplated it is important to give some insight into the sociohistorical context that shapes intensive care in New Zealand and internationally. This history illustrates the influence of the biomedical model on nursing practice within ICUs and demonstrates how nurses are still able to practice using their own philosophies and in a holistic manner. The wider contextual issues influence both nursing and medical practice in the critical care environment and assist to explain why sedation management is so complex. These contextual matters also provide justification for how nurses and doctors practice differently and why this difference may mean there is an impact on how each professional may assess sedation in the ICU. This chapter considers the role of nursing and technology in the ICU, the influences on clinical decision-making taking into account the impact of experience and nurses intuition, and provides examples of how nurses and doctors interact and use various health tools to inform their practice. It is important that these influences on clinical decision-making are considered before examining the role of sedation in the ICU as they may have a significant impact on how practice decisions are made by nurses and doctors, such as, in assessing patient's sedation and the resulting action of titrating of sedatives.

\section{The Socio-Historical Context of Intensive Care}

Specialist critical care units are a comparatively new phenomenon even though the importance of a nominated area for close observation of acutely unwell patients was initially recognised in the nineteenth century (Nightingale, 1863). As the advantages of concentrating the most seriously ill patients and skilled staff in one place became recognised, ICUs developed from the post-operative recovery rooms and respiratory units of the 1920s, 1930s and 1940s. The emergence of ICUs corresponded with the development of techniques of artificial ventilation, cardio-pulmonary resuscitation and increasingly complicated surgical and medical procedures (Seymour, 2001). There was also awareness that during the 
polio epidemic of 1948-1953 intermittent positive pressure ventilation (IPPV) along with a tracheostomy tube and a hand inflated rubber bag on an anaesthetised patient was a useful treatment for bulbar poliomyelitis. This innovative method of managing respiratory failure opened the operating theatre doors giving anaesthetists a new role in the care of the critically ill. This is regarded as the beginning of intensive therapy (Gilbertson, 1995). IPPV then replaced the previous ineffective attempts of introducing air into the lungs. Moreover, the new method was more flexible and user-friendly than the giant 'iron lung' used formerly to treat people with chronic respiratory failure (Trubuhovich \& Judson, 2001). Following the early success with IPPV it quickly became applied to thoracic surgery, general surgery, tetanus, and crush chest trauma. The movement of anaethetists from the operating theatres to the postoperative (recovery) environment was further stimulus for the growth of ICUs. The anaethetists' knowledge enabled the development of biochemical, haematological and physiological monitoring to an unparalleled extent.

In New Zealand the first 'respiration unit' was established in December of 1957 at Auckland Hospital. Inadequate breathing from poliomyelitis had been treated in New Zealand by ventilators prior to World War Two; however these were 'iron lung'ventilators. The first IPPV ventilator was not acquired until the late 1950s and was mostly used to manage patients during the poliomyelitis and tetanus epidemic. The founding Intensivists of New Zealand were infectious disease specialists with support from anaethetists (Trubuhovich \& Judson, 2001). ICUs then opened in other New Zealand hospitals over the following decade.

The first 'iron lung' was purchased for Wellington Hospital in 1938, for the treatment of poliomyelitis. Until 1962 Wellington had no recovery room or an ICU. Patients were recovered outside the theatre doors or in the wards. In 1963 Doctor (Dr.) Cam Barrett came to Wellington and was given the task of caring for various medical and surgical patients who required ventilation in wards scattered through out the hospital. There were two ventilators available for his use. He 
trained nursing staff to care for these patients in the wards until in 1964 the first ICU was opened with the support of a physician, a general surgeon and Dr. Barrett (Trubuhovich \& Judson, 2001). A nationwide Postgraduate Intensive Care Nursing Course commenced at Wellington Hospital in 1968. This recognised the pivotal role of nurses in intensive care. The nurses were lectured by medical staff from the newly emerging special units (renal, coronary care and cardiothoracic) and Dr. Barrett convened the course. Eight registered nurses from around New Zealand took the first six-month course. It has continued annually since, recently becoming a university advanced practice (postgraduate) certificated course.

The United States of America (USA) saw a more rapid and initial development of intensive care than was observed in other parts of the western world. This was in part driven by the USA health funding system of health insurance. The ready adoption of 'technologies of rescue' (new drugs and procedures for treating multiple organ failure and sepsis) (Reiser, 1992) in the USA resulted in the character of ICUs in that country varying from that of the United Kingdom (UK) and New Zealand. Firstly, units in the USA tend to be much larger. ICUs of twenty beds or more are the norm, while in New Zealand ICUs tend to be smaller, ranging from four to 20 beds. Similarly ICUs in the USA are more uniformly spread across the country. In New Zealand the availability of ICUs is not uniformly spread, although it is an established principal of the Ministry of Health that all hospitals have access to some form of intensive care facility. Another significant difference between New Zealand and other ICUs internationally is the character of the patient caseload. Seymour (2001) suggests the USA patient demographics show more variation than in the UK, with a trend of patients who are either far more or less ill than would be the case in the UK. It is not clear how New Zealand's ICU patient group compares internationally as no published benchmarking was located. Benchmarking is a relatively new concept and Capital and Coast District Health Board (C\&CDHB) has only recently started collecting data from a quality assurance point of view. 
One of the distinguishing characteristics of contemporary intensive care throughout the western world is the crucial role played by nursing staff. In New Zealand as in the UK, ICUs are staffed traditionally according to a $1: 1$ nurse to patient ratio, whereas in the USA the ratio is more variable. This is in part due to different supportive roles in the USA, for example, respiratory technicians who manage ventilation. ICU nurses work alongside medical staff in an uncommonly close and often interchangeable way in New Zealand, with few or no supportive technical roles.

Lynaugh and Fairman (1992) suggest that the ICU symbolises a contemporary preoccupation with the mastery of disease, the annihilation of an 'untimely death' and the prolongation of life. ICU has become the location to which physicians refer a patient when they stand at the verge of death and are past the scope of conventional treatment. Patients with progressively more complex surgical procedures have required admission to ICU as an essential and routine part of their post operative care. ICU also has a role to play in the palliative care of patients, in caring for patients with enduring conditions such as AIDS and invasive cancers. A spill-over effect of ICUs can now be seen with treatments that were once restricted to specialised units now becoming integrated into ordinary practice within hospital wards (Seymour, 2001), for example noninvasive ventilation techniques.

The type of patient admission to adult ICUs tends to reflect both demographic patterns of the country and the hospital population as a whole. A trend seen in New Zealand and internationally is the growing percentage of elderly intensive care patients suffering from an acute exacerbation of chronic illness, rather than from the consequence of an infectious disease or acute trauma. Despite the fact that the patient mix of ICUs may differ, there are tensions (which are perhaps a central feature of current hospital care) caused by financial limitations, demography and technology that are encapsulated in an acute way within the ICU. Here, patients who would have died previously from conditions may now 
potentially survive. This impacts on the resource allocation of health funding within a community. Nevertheless as yet, there is still an unpredictable and unknown patient outcome to much intensive care management (Audit Commission, 1999; Koch, Rodeffer, \& Wears, 1994; Ridley, Biggman, \& Stone, 1990). Broad debate has developed over the rationing of ICU services and being more efficient with the funding and technology available (including sedation). This discussion has developed alongside a parallel debate about the most appropriate ways of stopping the delivery of futile therapy. This debate will continue for some time as ethical principals are challenged and reviewed along with the boundaries of intensive care.

Today there is considerable diversity in the nature of ICUs around the world. Various ICUs include specialty units such as; neurosurgical, cardiothoracic, paediatric, neonatal, burns, trauma, coronary care, and more general ICUs such as surgical, medical and general ICUs. These vary depending on the population needs of the country. This study was undertaken in a tertiary general ICU that included specialties such as paediatric, cardiothoracic, neurosurgical, burns and trauma. The only other ICUs within Wellington Hospital were a neonatal unit and a high dependency coronary care unit.

Alongside the evolution of a contemporary critical care unit, to a large extent commentary focuses on the technological environment with its system of tools, knowledge and skills. Many of these commentaries in this fashion neglect nursing care, rendering the nurses work invisible or unacknowledged. The critical care nurse has an important role to play in how technology is managed and balanced with the human side of ICU. The nurse brings together the science and the art of nursing to provide a holistic focus to care in the ICU. The uniqueness of how the nurse provides this balance to nursing practice is discussed in the following section. 


\section{Nursing and Technology in Intensive Care}

ICU nurses have been criticised in the literature for being immersed in a biomedical model of practice in which the emotional and social nature of the nursing and patient experience are ignored or undervalued (Dean, 1998). As discussed previously, the first ICU nursing course run at Wellington Hospital was co-ordinated by an anaesthetic consultant and much of the nursing orientation to ICUs was biomedically based. However, today new staff to Wellington ICU have already had at least two years postgraduate experience and now come with a university based nursing education and knowledge underpinning their practice. This foundation provides some balance to the biomedical environment of the ICU they are entering. Seymour (2001) in her study views medicine as the context within which nursing practices and within which it must shape its relationships with patients and their families. Benner and Wrubel (1989) argued however, that nursing is dually constituted: first by the technical-medical needs and secondly by strategies that nurses use in ICU to integrate the 'whole person' into what may be viewed as a depersonalised circumstance. This is the science and art of nursing at work. Achieving and sustaining this balance is the core of nursing practice in ICU. In contrast, medicine is commonly described by its ability to separate the 'body' from the 'person'; furthermore the 'body' is then broken down into a set of medical problems. This fragmentation and depersonalisation of the body enables medical personnel to specialise in one specific attribute or system of the body. This defines each field of medical accountability and allows medical practitioners to manage and prioritise their complex responsibility (Seymour).

\section{The Two Discourses in Intensive Care}

There are two paradoxical discourses operating within ICU. Firstly the biomedical discourse. This incorporates the sciences such as the biological, physical and behavioural sciences, technology and curative therapies. This discourse values objectivity, and reductionism (Benner, Hooper-Kyriakidis, \& Stannard, 1999; Jenner, 1997). Smythe (1987) describes the biomedical discourse using the metaphor of the 'eyes and intellect' of nursing. That is, the assessment that the nurse utilises to draw conclusions based on his/her knowledge; these in turn lead 
to a nursing action. The second discourse, the human life world discourse is described by Benner et al. as the human experiences, intuition, expression, participation and values of subjectivity, creativity and holism. Smythe (1987) portrays this as the 'heart' of nursing. These commentators suggest that the union of the heart, eyes and intellect are regarded as essential for harmony in nursing practice. Neither discourse is superior they are different. Wajcman (1991) uses the term epistemological pluralism to define their relationship with the critical care nurse. This involves a coalition of the science of nursing with the humanism qualities; both should be acknowledged equally.

The biomedical and human life world discourses may appear diametrically opposed in an ICU and there are times when there may be conflicts between them. For example, during end of life care, where qualitative discussions arise about whether the situation is one of false hope or of effective yet futile treatment. Between these two discourses sits the nurse. Human-to-human interaction is the core of nursing practice (Johnson, 1996). What nurses try to avoid is the exaggeration innate in the extremes of both discourses and they attempt to work with what is real in each. In order to gain an understanding of the tension that exists between the two discourses a discussion on the tension between technology and the art of nursing in the ICU setting is presented.

\section{Technology and Nursing}

The technologic framework has its origins in western society's move from an agrarian to an industrial society. This model values exactness, knowledge, skills and outcomes; all of which are reminiscent of the industrial revolution. In contrast the humanistic framework is believed to date back to biblical times and values respect for individual's rights and contributions, collaboration, empathy and the person's emotional, spiritual and psychological needs (McConnell, 1998). Critics of the impact of technology in nursing (Bradshaw, 1998) claim that these frameworks are incompatible. Nowhere in nursing does this paradoxical relationship exist more than in the ICU. In this environment the nurse is the patient's bridge to the personal world within an impersonal network of 
technology. The nurse in an ICU facilitates the coalition of technology and humanism (McConnell).

Sandelowski (1998) affirms that the science of nursing is progressively more informed by post-modern tools, for example, cardiac monitoring, arterial blood gas machines and chest x-rays. These technologies can be perceived as a way of knowing in nursing. Nursing practice increasingly includes 'hermeneutic relations' with devices; this entails the nurse reading or interpreting data from devices and acting on this information. Sandelowski suggests this concept should be added to Carper's (1978) fundamental patterns of knowing and acknowledged as nursing informatics. Alongside the hermeneutic relations with devices, is the hermeneutic relationship that nurses have with their patients. This involves interpreting the meaning of being human which has always been a central issue to nursing (Welch, 1999).

A review of the literature by Dean (1998) pertaining to critical care nurses' relationship with technology has highlighted two main criticisms of the relationship. Firstly ICU nursing practice in a high technological environment is taking on a 'curative' role and is working within the boundaries of the biomedical model. Secondly, the technological environment may dehumanise the patients and potentially the families and nursing staff. These criticisms certainly paint ICU nurses in a poor light whilst nurses are supposed to 'care' not 'cure'. Historically, curing has been associated with the realm of medicine, while caring was solely a nursing role. All too often technical expertise is seen in the literature as being 'medical', 'scientific' or 'curative' (Hogg, 1994). Considering this within the context of sedation management an extension of the nurses' role becomes evident. In the past the nurses' role of caring for the patient would not have involved titrating sedatives or making clinical decisions based on a sedation assessment, while today this has become a common part of their practice in ICU. Nurses do manage technology and science as part of their practice and work collaboratively with medicine to ensure care is appropriate and timely. Medicine relies heavily on nursing within ICU to carry out technical and scientific assessments and care. 
In contrast Ray's (1987) model of technologic caring in ICU, equates caring with technology. The model she presents involves a hermeneutic nurse-machine relationship. This relationship takes account of interpreting monitors, numbers, lines and tubes, acting on that interpretation within the context of a nurse-patient relationship. This is because the ICU nurse looks beyond the technical to being 'with' the patient and knowing them hence the technology works for that patient to achieve the best outcome for the individual. Benner and Wrubel (1989) and McConnell (1998) also support Ray's model by suggesting that the ICU nurse melds technology and humanistic values. This integration can only occur because of the nurse. The nurse has the knowledge of the individual patient, the knowledge of their self as a nurse, and the knowledge of technology. Critical care nurses cannot control who lives or dies, nor do they control a person's will to live, however, they do have the knowledge, ability and desire to make a difference in someone's life. This does not come from a machine or science; it comes from the very foundation of nursing's practice and knowledge, that is, understanding the human experience.

In most situations technology does not 'cure' it only provides a tool, for example, to monitor physiological factors. It is a tool that nurses use just like they use a nursing model as a tool. Technology may extend the abilities of the nurse but it is no substitute for the nurse. The nurse is not dependent on technology to deliver care. As McConnell (1998) states,

"This is absolutely critical for it is of no value to be a humanist and watch a man die for lack of technology nor is it of any value to be rich in technology only to watch a man live and die without dignity" (p. 27).

This statement illustrates the epistemological pluralism inherent in ICU nursing. Much of the technical and theoretical knowledge ICU nurses have, comes from the integration of scientific knowledge with other nursing attributes, including wisdom, experience, ethics, intuition, caring and human spirit. These are all different and should be equally celebrated as they are all needed to render technical procedures tolerable, acceptable and safe for patients. 
In 1991, Wajcman wrote a feminist critique of technology. In this account she concludes that females have a different style of using technology from males. Females tend to have an interactive, relationship style, in which they reshape the technology to accommodate themselves and their world. The same can be argued for ICU nurses' relationship with technology. In fact Wajcman identifies nursing as an example of this interactive relationship. In comparison, Wajcman suggests that males tend to impose their will and try to control the technology. Naisbett's theory (cited in Dean, 1998) of 'High Tech, High Touch', is also relevant to the care of the critically ill and how nurses interact with technology. The theory suggests that in introducing new technology to society, it requires a counter-balancing human response; otherwise the new technology may have adverse effects. The ultimate goal is for technology and nursing to work in harmony. There is a deep-seated ethic in critical care nursing of protecting patients and humanising the environment as the use of technology expands. Ensuring a safe environment in an ICU setting is no small achievement. Benner and Wrubel (1989) suggest nurses protecting the safety of their patients is a form of caring.

While caring practices are perceived as artful, they are also knowledgeable and lifesaving. Articulating the knowledge embedded in caring practices brings them in from the margins. If we take the example of a trauma patient; people immediately focus on the 'beeping' of the ECG monitor, the ventilator noise and the bloodied face. Few notice the fundamental intensive nursing care, solving daily problems of living, pain, providing comfort, preventing and treating pressure or mouth sores and promptly recognising and responding to infection. If these simple measures of mouth, skin or comfort cares are overlooked, they can lead to complications which may prolong a hospital stay and patient suffering or even trigger a cascade of events that eventually leads to death (Benner et al., 1999). Nurses in ICU do carry out additional tasks, such as, defibrillation, managing ventilation and titrating sedative infusions. These technical skills should be seen as an extension of their caring not a diminution of nursing care. Indeed if the 
trauma patient died, the family left behind is more likely to value the human-tohuman interactions that are nurses not buttons, alarms and machines.

Critical care nurses work in a collaborative relationship with medical staff. In nursing practice each day decisions are made about patient care. Some in consultation with medical staff and others are nursing decisions. How nurses make clinical decisions in an ICU is also of interest to many commentators. Because of the very nature of the ICU, the decisions that are made have a significant impact on the patient, their family and the course of their stay. Before considering how sedation in ICU is managed it is important to appreciate the complexity of nurses' clinical decision-making in the critical care environment.

\section{Factors Influencing Clinical Decision-Making in Intensive Care}

The ability to think critically is a fundamental principle guiding nursing practice today. The ability of a nurse to make appropriate assessments, to identify potential patient problems, intervene appropriately and to prioritise care all necessitate decision-making skills. Clinical decision-making takes place when one course of action is chosen over alternatives, and critical thinking is a necessary element of clinical decision-making (Gerdtz \& Bucknall, 1999). The terms clinical decision-making and clinical judgment are frequently used in the literature interchangeably (Baker, 1997). Clinical decision-making in contemporary nursing practice is considered to be one of the most critical clinical functions undertaken by a nurse. It is an intrinsic part of clinical practice and is the central skill that distinguishes expert nurses from inexperienced nurses.

Two theoretical positions that describe how nurses apply judgment when making clinical decisions are presented in the literature; the rationalist perspective and the phenomenological perspective. The rationalist approach describes decisionmaking in terms of diagnosing a problem, implementing a treatment and evaluating the outcome. This approach removes contextual features from decision-making. It ignores the dynamic nature of clinical practice and the impact of those dynamics upon real clinical decisions and it requires a clearly defined 
relationship between the decision and the outcome (Bucknall, 2000). In contrast the phenomenological perspective endeavours to address the shortfall in the rationalist perspective by describing the context of decision-making in clinical practice (the natural environment). Personal and professional values, intuition, cognition, insight into the whole situation and the context of the practice setting are regarded as fundamental influences that shape clinical decision-making (Gerdtz \& Bucknall, 1999). Several qualitative studies have described the role of intuitive knowledge in a nurse's decision-making and identify pattern recognition as a significant factor in clinical judgment in nursing (Benner, 1984; Benner \& Tanner, 1987). A combination of both the qualitative and quantitative research (underpinned by a fusion of the two discourses) on clinical decision-making in nursing is now becoming apparent, as is demonstrated by Bucknall. This synthesis of approaches is also seen in clinical practice with the use of health care assessment tools. Health care tools or scales provide a standardised approach of expressing a decision in relation to a treatment concern. These tools, such as sedation-scoring tools, assist the nurse to arrange assessment data and describe the individual patient's need for a specific care in a consistent manner, at the same time nurses' take into consideration contextual influences when making a judgment on the most appropriate course of action for a patient in conjunction with the tool.

\section{Intuition}

Clinical knowledge is developed over time from multiple practice experiences. From their practice experiences expert nurses are able to identify subtle variation in a patient's physiological state; they can identify an imminent critical episode before any parameters have altered. This feature of expert nurses is described by Benner and Tanner (1987) as intuition and is defined as "understanding without a rationale" (p.23). This understanding is not accidental or guessing; Benner and Tanner advocate that intuition is a valid and a fundamental aspect of clinical judgment. Integrated in Benner and Tanner's model of clinical judgment are Dreyfus' six key characteristics of intuitive judgment. These comprise of pattern recognition, similarity recognition, common sense understanding, skilled 
know-how, sense of salience, and deliberative rationality. Benner and Tanner suggest these characteristics are essential and work together mutually for expert nursing judgment to occur. Although intuition may contribute to decision-making by experienced nurses there is more that leads the expert nurse to make a clinical decision. The knowledge and experience nurses have acquired through specialty practice also informs nurses' decision-making. Intuition is therefore only one facet of clinical decision-making.

Benner et al. (1999) suggest that expert critical care nurses handle a vast array of information at any one point in time, however are still able to centre on the most relevant findings, changes, or issues. They explain this by reference to the notion of clinical forethought which enables the nurse to anticipate a likely clinical event and to take the necessary appropriate action. This is formed from the nurses' clinical understanding of the situation. Clinical forethought is seen to guide thinking-in-action and is rooted in particular clinical situations that develop into a habit of thought in that the nurse recognises similar patterns in the future. Benner et al. describe this as a significant component of clinical decision-making. This is the aspect of nursing knowledge and practice that is often invisible to the observer who is trying to define and contextualise decision-making in nursing.

\section{Contextual Influences on Decision-Making}

Benner's From Novice to Expert (1984) framework assists us to appreciate how nursing knowledge becomes embedded in practice. This knowledge is gained over a period of time and with exposure to practice experiences contributes to expert nurses' ability to perceive subtle changes in their patients' and act upon that information appropriately. Novice and advanced beginner nurses' are not able to inform their clinical decision-making with these judgments as they have little knowledge and experience of the situations they are practising in. Therefore a system of rules and guidelines based on their nursing education shapes their practice. In comparison the expert nurse does not solely rely on guidelines and policies they have assimilated to guide clinical decision-making in the practice setting because they utilise the breadth of their nursing experience and 
knowledge. Nursing experience is not defined by time spent in practice. Benner portrays how Gadamer observed that nurses refined their early nursing knowledge and principles through their clinical experience and these experiences enrich and rework their nursing theory and practice. A nurse gathers a wealth of experiences and applies this amassed knowledge to new situations (Benner). Whilst a systematic, consistent approach to sedation assessment is important in managing critically ill patients it does not negate the experienced nurse's intuition. These tools actually support a nurse's use of intuition in that they work jointly with the knowledge of assessment and experience of the nurse to guide practice decisions. Even sedation assessment tools can be flexible enough to accommodate the knowledge and experience of the individual nurse.

An early study by Bakken Henry (1991) attempted to examine the effect of patient acuity on clinical decision-making practices of intensive care nurses with varying levels of knowledge and experience. The findings supported the notion that both the nature of the task and the characteristics of the decision maker influenced decision-making. This was further supported by Bucknall and Thomas (1997) who found that facets of the environment in which the decision-making was taking place were influencing critical care nurses' decision-making. These included; patient complexity, physical structure of equipment and social interactions.

A later study by Bucknall (2000) also described the decision-making activities of critical care nurses' working in clinical practice. This study indicated that individual and environmental variables influenced nurses' clinical decisionmaking. In particular the number of decisions nurses appeared to make was associated with nurses' critical care experience, position level, ICU location, and shift work. What have yet to be determined, however, is whether the contextual influences on nurses' decision-making has an impact on patient care outcomes. Often these are more difficult to observe in studies that just take a snapshot of nursing decision-making; contextual influences can then be missed or their importance in decision-making may not be fully appreciated. In considering 
clinical decision-making's impact on patient care outcomes, it is important to also consider that a certain amount of decision-making that occurs involves collaborative decisions with other multidisciplinary professionals rather than individual ones. Accordingly further studies are needed to compare the process and outcomes of collaborative decision-making as well as those by individual nurse's. Sedation assessment and management in critically ill patients is often based on collaborative decision-making between the nurses and doctors the process involved in this decision-making has not been discussed readily.

Benner et al.'s (1999) study builds on Benner's (1984) earlier work by providing a window into the complexity of nursing care provided in critical care units and the decision-making encompassing it. They describe a thinking-in-action approach to clinical knowledge and interventions in critical care. This work further ascertains that expert nurses' clinical decision-making has always been positioned within their practice context. Benner and colleagues' work has moved from an emphasis on defining a problem, to the problem-solving processes. They identify nine domains of practice in critical care nurses that uncover aspects of clinical judgment and clinical knowledge development. These are; diagnosing and managing life-sustaining physiologic functions in unstable patients, the skilled know-how of managing a crisis, providing comfort measures for the critically ill, caring for patients' families, preventing hazards in a technological environment, facing death; end of life care and decision making, communicating and negotiating multiple perspectives, monitoring quality and managing breakdown, and finally the skilled know-how of clinical leadership. Benner et al. argue that these domains reveal how being located in a clinical situation guides clinical decision-making and the resulting action. They also take the position that clinical decision-making requires more than placing information into distinct categories and although critical care requires precise judgments, clinical decisions cannot be as certain and controlled to the extent that scientific experiments can. 


\section{Decision-Making Behaviours in Intensive Care}

Although there have been changes in the level of nurses' participation in decision-making activities, the changes have time and again been slow and variable between practice settings. Some studies have established that nurse decision-making and autonomy is a central determinant of job satisfaction (Alexander, Weisman, \& Chase, 1982; Slavitt, Stamps, Peidmont, \& Haase, 1978). A 1991 study by Stern et al. found that nurses in a Paediatric Intensive Care Unit (PICU) supplied a significant amount of information but were unlikely to be decision-makers. In comparison medical staff were perceived to be high frequency decision-makers. These factors may also contribute to job satisfaction. In contrast, Bucknall's (2000) observational study of clinical decision-making behaviours in critical care nurses found that $80 \%$ of critical care nurse decisionmaking was in the areas of communication and evaluation and the remaining $20 \%$ was interventional based. In this study Bucknall also noted the high numbers of decisions made by nurses and the rapid pace that was required in nurse decision-making. This study clearly indicated that individual and environmental variables influenced nurses' clinical decision-making in a critical care unit. The disparity between the two studies may be explained by the different approaches to defining what a clinical decision was.

\section{Environmental Factors}

A recently published study by Bucknall (2003) sought to ascertain the environmental influences on nurses' decision-making in the ICU setting. Bucknall suggests there is a substantial gap in the literature considering the influence of the clinical environment on nurses' decision-making. This is also alluded to by Benner et al. (1999). Much of the literature discussing clinical decision-making is 'a-contextual'. As Bucknall affirms clinical decisions are not activities that occur in isolation, but rather incorporate the clinical context of political, economic, ethical, legal and social structures. Within ICU technology, staffing workloads and role definition are important aspects of practice that impact on the decisions nurses make. Bucknall established three core groupings 
of environmental influences on nurses' decision-making; patient situation, availability of resources and interpersonal relationships.

The patient situation or complexity of the patients was a significant influence on nurses' decision-making. The characteristics of a patient's condition influenced the type, rate and complexity of the decisions. If time was not a pressure, unfamiliar patient diagnoses made nurses more conscious of their actions (in terms of slowing the process) in considering the best option for their patient. Bucknall (2003) also found that nurses were more at ease making clinical decisions when they could discuss these with colleagues or had the time to consider them in the context of previous experiences.

The second category identified by Bucknall (2003) was resource availability; both the physical layout of the unit including the equipment and staffing resources. The physical arrangement of the ICU has a bearing on stress levels along with the availability of resources. These impact on nursing workloads and the quality of patient care. Bucknall found that nurses working in isolation rooms considered themselves to be more autonomous in their decision-making because of the structural difficulties in communicating with senior staff. Nursing staff also identified that decision-making workloads increased appreciably when there were fewer experienced nurses on duty. Nurses were expected to assist less experienced colleagues even if the experienced nurses had more complex patients. Staffing resources also influenced the conventional boundaries of nurses' decision-making. ICUs that relied heavily on casual or agency staff and those with no on-site medical staff at night had the boundaries of nurses' decision-making expanded.

Finally Bucknall (2003) established that interpersonal relationships in critical care were a crucial component of multidisciplinary patient management. The collaborative relationships with team members are essential for the running of the ICU and the care that is provided. The relationship in particular between nurses 
and doctors is crucial. This relationship is unique and not often seen in other areas of a hospital.

\section{The Relationship between Nursing and Medicine in Intensive Care}

The critical care environment encircling seriously ill people can be viewed as like being on 'fast forward'. Within a time span encompassing only a few hours or days, people are admitted, examined, diagnosed, discussed and cared for until their death or recovery. Multi-professional activity, during this period can be frantic and fast moving; clinical knowledge and decisions must be formed about the critically ill person; relationships created and maintained among the ICU staff, patients and their families. Furthermore the technological environment of critical care and its relationship to the rest of the (fragmented) hospital organisation influences the multidisciplinary roles of the staff working within this environment.

The Nature of Nursing and Medicine in Intensive Care

Within the ICU nurses and doctors work collaboratively to plan and provide care for critically ill patients. Many definitions of collaboration assume that the power relations between individuals are equal (Manias, 1998) however in considering the nurse-doctor relationship definitions of collaboration often do not recognise the existence of unequal power relations. To develop collaborative associations and overcome the inequality in the relationship between nurses and doctors requires efforts from both professions. These efforts entail a mutual respect and understanding for each profession. The current nature of this relationship may well influence how sedation is assessed and managed in the ICU.

The doctors and nurses in ICU practice 'side-by-side', both giving guidance, expressing opinions, beliefs and frustrations, at the same time both professional groups engage in the many physical tasks involved in a patient's management. Nonetheless on closer investigation their roles are arranged quite differently. Medical staff have responsibility for initiating changes in treatment, ordering 
investigations and diagnosing the numerous complications that all patients in ICU may develop (Seymour, 2001). The nursing staff, in contrast have responsibility for making sure the various treatments are carried out safely and precisely and that the physiological information on which therapy is based are recorded and reported appropriately. These events are carried out alongside the tasks more usually associated with nursing: washing, turning, clothing, and redressing of wounds. Moreover, this care is coupled with the giving of reassurance to patients and the judgments as to their need for comfort, sedatives, and analgesia and anticipating potential problems which may occur. ICU nursing staff also have principal responsibility for providing support, comfort and information to a patient's family; this tends to be done primarily in an informal manner, integrated into nurse's work of caring for the critically ill patient (Seymour). Nursing staff are usually responsible for one patient on their shift. They are able to spend a significant period of time getting to know that patient as they work with them.

The medical staff make contact with patients and their families in a comparatively sporadic fashion. In Wellington ICU for example, the 12 medical staff have equal responsibility for all patients in the ICU and have to share their attentions between them all. Stress and tension can arise because of these differences between the responsibilities of nurses and doctors in ICU. While the nurse is expected to concentrate on one critically ill patient at any given time, the doctor must be available for all patients. This can create tension between the roles. These differences in roles may mean nurses and doctors evaluate patients' sedation needs differently and have a different understanding of the language used to describe behaviour influenced by sedation.

In Seymour's (2001) study, descriptions of 'nurses' work' provided by nurses in critical care units was noticeably different to the descriptions provided by the medical staff. Nurses acknowledged the medically-oriented aspects of their intensive care nursing practice, but they positioned these activities within a context of an emphasis upon the physically private and continuous features of 
their involvement with patients as well as the course of building a close relationship with their families. The multidimensional nature of nursing in critical care and the nurses intimating the discourses of 'holism' and 'total patient care' indicates that nurses' perception of the patients were skewed away from the medicalised stance which doctors tend to engage in. Nurses tended to describe their patients as individuals they knew well, who had personal styles of response to therapy, which were not exclusively dependent on objective, measurable data (Seymour). These descriptions are often heard in the conversations nurses have even though the patients are unconscious and have never been known as conscious persons to the nurses caring for them. In this way nurses strike a balance between the various parts of their role; they collaborate with the technical management of their patient's body but they also represent the bodily response in ways that ascribed subjectivity to the unconscious patient. Accordingly the medicalised nursing work is tempered with nurses achieving interactive, intimate relationships with their patients and their families. This is taken up by Lawler (1991) who considers that nurses still live up to the discourse of holism in contemporary society: "one cannot simply nurse the body in the bed. One must do business with it as a person because nursing means being able to view the body and the person as inseparable" (p.34).

Current debates about the nature of nursing work have depicted the evolution of a new relationship between the nurse and patient (Salvage, 1990). The centre of this relationship is perceived as an emotional exchange and development of personal knowledge about a patient by his or her nurse. Caring 'for' a patient, in a practical, visible, and task oriented sense has been exchanged in nursing by the language of caring 'about' in an emotional, private, essentially hidden and person-centred manner (Benner et al., 1999). Field (1989) has revealed how the 'comfort' and 'sentimental' features of nursing practice are rooted within the more observable activities of technology, safety and communication work associated with the medical treatment of patients in ICU. ICU nurses are presented with the opportunity of associating themselves with the technological and diagnostic 
competencies rather than the person-centred caregiver, and it is this aspect of their role that is recognised by medical colleagues.

"In ICU nurses have developed skills and accept responsibility for areas of treatment management that at one time would have been unquestionably within a medical domain. Consultants and ICU support the development of nursing skills, and set parameters within which nurses will decide on levels of sedation or assess change in ways that appear strikingly similar to making a diagnosis, but this is perceived as part of a process of providing care" (Walby \& Greenwell, p.42).

Reliable sedation-scoring tools are able to objectify some of the subjective behaviour of patients. Nursing staff are then able to use these tools to inform their practice decisions in a way that is still appropriate for individual patients in a timely manner. Nurses, on the one hand, have increased autonomy and are respected by their medical colleagues because of their knowledge and orientation and on the other hand, they still manage to practice from a nursing base, taking into consideration the 'whole patient' and their family's needs.

Svensson (1996) suggests that the complexity of, and overlap between nursing and medical practice in critical care is such that the relationship between doctors and nurses is no longer an issue of dominance and hierarchy in which medicine defines nursing. Rather, negotiations are undertaken during the clinical practice interface, which construct the very nature of nursing and medical practice. Both the nursing and medical domains are shaped by their interactions in practice. How these professions are defined is largely an issue determined through negotiations about the division of work on the ward (Svensson). In ICU nurses and medical staff actively engage in negotiations as part of their clinical practice. The negotiation that takes place and the shifting of boundaries does at times give rise to conflict. Many ICU nurses identify conflict and power as significant roadblocks to the interpersonal relationships and decision-making that takes place (Bucknall, 2003). The relationship between nurses and medical staff in ICU is evolutionary in nature, not static. 


\section{Power and Conflict in Intensive Care}

Traditionally the more advanced levels of autonomy and responsibility were frequently assigned by medical practitioners. In ICU because of the close proximity of the medical and nursing professions there is potential for conflict and blurring of the boundaries which define their roles. Bucknall and Thomas (1997) suggest there is little wonder that conflict arises between these two professions in this environment given the significant variability in decision-making behaviour. This is also influenced by the difference in protocols and procedures between national ICUs, and the occasional presence of vague legislation governing the actions of medical and nursing professionals that requires clarification.

The nature of contemporary intensive care requires a collaborative team approach to patient care. However role definitions and the power base in ICU has its origins in traditional and historical boundaries that continue to exist. The distinctive practices of medicine and nursing in ICU were easily identifiable thirty years ago. The contemporary role of the ICU nurse has now evolved with additional responsibilities from the medical profession (role extension) and the development of nursing (role expansion) through improved nursing knowledge (Coombs, 2003). As critical care knowledge has evolved there is greater understanding of what is happening to the patient and they have been stabilised and managed in a timely fashion. This knowledge becomes embedded in practice and consequently the boundary between nursing and medicine becomes distorted. Instances of nursing's role change in ICU are presented in the literature, for example, the development of nurse-led patient weaning and extubation programs (Anderson \& O'Brien, 1995), new assisted ventilatory modes, sedation administration, and drug and fluid prescriptions (Department of Health, 2001; Royal College of Nursing, 1997; Brook et al., 1999).

The ICU is a multifaceted area of health care involving high levels of uncertainty and instability. Collaboration between the professions is crucial to effective patient outcomes in this area. Some studies have established instances of contested inter-professional boundaries within critical care (Allen, 1997); few 
however have substantiated inter-professional conflict and negotiations. A recent study by Coombs (2003) considered medical and nursing staff's perspective on clinical decision-making in order to consider contemporary clinical roles in critical care. A significant matter arising from this study was that although the critical care nursing role had changed this appeared to have made little impression on how clinical decisions were made. Despite the advent of advanced nursing practice, Coombs argues that nurses still remain largely unseen within intensive care decision-making.

Medical and nursing staff equally acknowledged conflict during patient management discussions, nonetheless it was principally nurses who sought to redress this through developing specific behaviours in the clinical environment. The study by Coombs provides illustrations of situations where nurses 'play the game' in order to participate in ICU decision-making. The main rule of the practice 'game' is that open disagreement between the participants is to be avoided at all times therefore nurses learn to communicate their recommendations in a manner that does not appear they are making them (Stein, Watts, \& Howell, 1990). Coombs provides practice narratives that draw attention to the hierarchy and the subtle power play that occurs during ward rounds, for example the physical positioning of oneself at the bedside and how nurses approach individual consultants. This behaviour has great implications for the collaborative team approach in intensive care as it provides little possibility for nursing to influence the patient care decisions made. The outlook for an effective ICU team lies with all professions realising the innate power in the complementary knowledge and roles held by each profession.

\section{Nurse-Doctor Interactions in Intensive Care}

The ward round is recognised as an important period for health care professionals to collaborate in developing an integrated plan of care. The goals are to improve the quality of patient care, share information, focus on patient problems, plan and evaluate complex treatment, and increase learning opportunities for staff. An effective relationship between health care professionals 
during the ward round is consequently an important requirement for improving the quality of patient care and decision-making. Despite an acknowledgement of this in the literature (Manias, 1998; Manias \& Street, 2001a; Whale, 1993) there has been only a narrow emphasis placed on interactions between nurses and doctors during the ward round. Studies concerning ward round nurse-doctor interactions have identified nurses' passivity and their lack of poise in asserting themselves in discussions (Whale). Manias and Street's study explores the power relations associated with the way critical care nurses interact with doctors during ward rounds. They uncovered complex power struggles that characterised nurse-doctor interactions during the ward round. They also found that nurses' involvement and communication throughout the ward round was reactive, which was in contrast to the proactive position of consultants. This may have serious implications for patient care and in particular in discussing goals for sedation.

The physical space of the ICU impacts also on how clinical decisions are made. Decisions made away from the bedside tend to be based on objective assessment of blood results or observational data, whilst more subjective concerns would be discussed at the bedside (Manias \& Street, 2001a). Nurses that could not walk away from the bed space to discuss subjective matters with medical staff would find these often overlooked in favour of more objective concerns in patient management decisions.

\section{Policies and Protocols}

Policies, guidelines and protocols are used in many ways to guide clinical practice and advance quality patient care (Manias \& Street, 2000). In the hospital setting, they provide information on the operation of technical equipment, drug preparation, care of patients with specific conditions, safety procedures, as well as providing guidance for health practitioners. Guidelines, policies and protocols provide some consistency to clinical practice and attempt to ensure a certain level of patient care is maintained while at the same time allowing for nurses and doctors to take responsibility in using their clinical judgment (Manias \& Street). 
The degree nurses and doctors use policies, guidelines and protocols to inform their knowledge in decision-making is not fully known. Little published material focuses on the diverse values attributed to nurses and doctors in their use of policies, guidelines and protocols to inform their clinical practice. The study by Manias and Street (2000) explored the way in which nurses used policies and protocols as a tool in communicating with doctors and their nursing colleagues in the ICU. Their study identified that nurses and doctors placed different values on policies and protocols to substantiate their knowledge. This difference was also dependent on the experience of the nurse and doctor. Less experienced nurses tended to be dependent on written guidelines and turn to them as an essential basis of their knowledge. Many nurses used guidelines, policies and protocols to justify their decision-making, while more experienced nurses would assimilate these guidelines into their practice but they were not the sole basis of their knowledge and clinical decisions. Policies, guidelines and protocols may offer a further way to affirm nurse's power in decision-making; they help to validate their decisions and bring additional substance to their interactions with medical registrars (Manias \& Street).

In comparison doctors were less inclined to place value on policies and protocols instead valuing their authority and autonomy. They would utilise their past experiences and background to inform their knowledge and support their decision-making activities. In the ICU context, I have observed registrars' decision-making is more likely to be influenced by past experiences and past consultants; where as more junior medical staff (novice practitioners) tend to rely heavily on guidelines to support their practice, this appears to have similarities with nursing. Nevertheless consultants in the ICU, who were also aware of unit policies and understood their value, would often back concerns raised or treatment decisions made by the nurses over those of the registrar. In this way the consultants acknowledged and gave value to the policies and protocols in the ICU (Manias \& Street, 2000). 
Manias and Street (2000) also suggest that policies and procedures constrained nurses' knowledge by restricting the type of knowledge nurses use to justify their practice. This is a concern if nursing becomes so reliant on policies and protocols, that future generations of nurses are not able to use the breadth of their nursing experience and other forms of knowledge to support their clinical decision-making. Nursing's voice may become silent. The danger lies in nurses that can only articulate knowledge which underpins their nursing judgments has come from one source, that of policies, guidelines or protocols (Buresh \& Gordon, 2000). The normalisation of policies and protocols that has been demonstrated by Manias and Street may constrain nurses' knowledge and practice development. Expert nurses tend to integrate policies and guidelines into their practice but rely less on them to make decisions every step of the way. They also base their decisions on a more profound understanding of the total situation, their vast background of clinical experience and their intuitive grasp of a situation (Benner, 1984). If the health care system becomes too rigidly regulated by policies and protocols these expert nurses may then be lost to nursing in the future as nurses' knowledge and practice becomes contained. While the Sedation-Agitation Scale (SAS) is not a policy, guideline or protocol it is a systematic way of assessing the impact of sedation using a set formula and may be used as a basis for developing sedation guidelines for nursing practice.

\section{Medication Order Charts}

Different modes of communication are used by nurses and doctors when they interact, including various forms of documentation. These include observational records that nurses gather as data for use by themselves and medical staff, blood result sheets, nursing reports and medication charts (Manias \& Street, 2001b). Manias and Street considered how nurses and doctors communicate through the medication order chart in the ICU. Medication prescriptions often involve clearly defined roles/tasks by doctors and nurses. Nurses are the professionals who organise and administer medication, whereas the doctors are responsible for choosing the appropriate medication and prescribing it; however there is an expectation that nurses remain well-informed of the clinical and legal 
issues surrounding the documentation of medication orders. Within the critical care environment these boundaries have become blurred.

From my experience prescribing responsibilities may be challenged by ICU nurses to ensure, in the best interest of the patient, that safe medication practices are followed and documented. It became apparent in Manias and Street's (2001b) study that regardless of doctors' legal right and responsibility for prescribing medicines, their medication knowledge (in particular that of new registrars) was not on a par with experienced ICU nurses knowledge of medicines. Knowledge of pharmacology and legal authorities created significant friction between nurses and doctors in the critical care environment. Experienced nurses were observed to coach doctors in the medication knowledge required for managing critical care patients (Manias \& Street). This knowledge used by nurses to alter a course of therapy remains invisible to the written information documented on the limited space of the medication chart. From my personal experience if you stand in a critical care unit and listen to the conversations taking place, you will hear nurses suggesting possible treatments to registrars including medication options. These remain invisible because the prescribing responsibility remains with the doctors.

Manias and Streets' (2001b) analysis of their observations resonates with my own practice. Experienced ICU nurses subtly involve themselves in the practice of prescribing through coaching of registrars whilst less experienced (novice and proficient practitioners) will accept the guidelines set without question or further consideration of the context. The ICU environment necessitates the need for nurses to be flexible in the care they provide, especially in the management of sedation. Nurses create that flexibility in their practice and patient management by coaching registrars in prescribing practices. Medical registrars rotate through the ICU and are not continuously present at the bedside because of the demands of the unit. The nurse remains at the bedside managing a range of clinical issues consequently nurses require flexibility in the guidelines they work within to provide appropriate care that is responsive to changing patient needs. 
Accordingly critical care nurses must concede that their role surpasses the traditional role of medication administration and legitimise this role extension by documenting and disclosing this as part of their practice (Manias \& Street).

Manias and Street (2001b) also observed examples of nurses regularly participating in self-policing and surveillance mechanisms in medication management. This was surveillance of both doctors and other nurses. Nurses generally censure colleagues for administering an inappropriate drug during double-checking procedures, during inspection of drug charts at bedside handovers, and during quality assurance audits. This is to ensure patient safety is paramount; furthermore it is a means of exercising power in their relationship with doctors and other nursing colleagues.

\section{Conclusion}

In the fifty or so years since ICUs have opened their doors to care for critically ill patients, they have evolved into the most technologically, highly developed environments in acute hospital facilities. Critical care has developed as innovation in technology and the ability of medicine to treat and support disease, has occurred. These developments have brought with them their own set of problems regarding the rationing of health services and the appropriateness of the care that can be provided. These will continue to be debated.

The very nature of intensive care nursing is one of managing risk and hope. Critical care patients are seriously ill and generally decidedly unstable. Their labile condition often demands decision-making from nurses in stressful situations and within a short time frame. Nurses use various forms of knowledge as a foundation for their decision-making. While integrating the biomedical model into their practice and decision-making, nurses also use knowledge developed from their own practice experiences and education to inform their decisions. How nurses make clinical decisions is also influenced by their nursing experience and environmental variables. Less experienced nurses tend to use guidelines and policies to guide their practice decisions as they have limited knowledge 
embedded in their practice on which to base decisions. Alternatively more experienced nurses whilst integrating guidelines into their practice also use the breadth of their nursing knowledge and other contextual factors to base their practice decisions upon. These factors may in turn influence how nurses make decisions about sedation management and possibly it may mean that less experienced nurses sedate patients more heavily whilst experienced nurses do not.

Nursing in ICU has a unique shape. It is dually constituted; it achieves and sustains a balance between the technical-medical needs of the critically ill patient along with the integration of holism. There remains an inherent ethic in intensive care nursing practice of protecting patients and humanising the environment as the use of technology expands. The technological nature of critical care nursing complicates decision-making and increases the decision-making responsibility of nurses; furthermore the advancing technology has challenged the traditional knowledge domains of nursing and medicine. As a result nurses now take on more responsibility for the assessment and management of patients' sedation. This could be perceived as nurses making a diagnosis and implementing a therapy.

Patient care in the critical care environment is generally a collaborative approach between multiple professionals. Multidisciplinary teams work in an environment where there are constant encounters with life threatening situations. Decisionmaking is therefore dynamic and changeable. Because of the close working proximity of the nurses and medical staff in ICU their role boundaries are constantly being redefined. Nurses move between the spheres of nursing and medicine as they integrate a holistic vision of care for their patients' and their families in ICU. Nurses and doctors practice differently in an ICU and it is important to consider these as they may in turn influence how nurses and doctors then evaluate patients' sedation needs and make practice decisions. Even if nurses and doctors use different decision-making skills when evaluating patients 
sedation in using the SAS what is important is that the final assessment is the same.

Now that the nature of the ICU and the macro-issues affecting the ICU and nurses decision-making are apparent it is important to consider in more detail the nature of sedation in ICU and why it can be so problematic for the ICU team. 


\section{Chapter 3- Sedation in Intensive Care}

The very nature of the Intensive Care Unit (ICU) environment makes decisionmaking complex and at times rapid. As Chapter Two demonstrated there are many contextual factors which influence this decision-making including the advancing technology of the ICU. A fundamental element of nursing care in ICU is providing optimal comfort to patients. The technological nature of the ICU is stressful for both staff and patients and sedation becomes an important tool to maintain comfort and reduce their risk of harm. Stress is usually considered a 'normal' compensatory mechanism, however, in the critical care environment it is coming to light that it can have significant adverse effects on already markedly compromised patients. Sedation is crucial to the management of these patients, however assessing the need for sedation in critically ill patients is very complex and due to the unstable nature of patients there is a fine balance between over and under sedation.

This chapter identifies the place of sedation in the ICU. The aims of sedation are identified along with how it works, the general sedation management strategies employed in ICU and why it is so important in considering the stress response of patients. The risks and benefits of sedation are discussed before examining closely how nurses assess sedation in patients and what influences that assessment. This chapter clearly identifies the difficulty and complexity in assessing and managing sedation in critically ill patients. The New Zealand context is discussed with regard to nurse-prescribing as the boundaries between nurses and doctors are blurred and being redefined within the ICU. Because of these factors which influence the management of sedation in ICU several sedation-scoring tools have been developed to help assess the need for more or less sedation in an individual patient. The tools also help to create some uniformity in sedation practices and common understanding between nurses and doctors in discussing sedation for their patients. The Sedation-Agitation Scale (SAS) developed by Riker et al. (1999) is the focus of this study and a description of the scale is presented. 


\section{Aim of Sedation}

The aim of sedation is to relieve anxiety, discomfort, aid treatment and enable nursing care. There is heightened attention today on the promotion of patient comfort, driven by evidence around the adverse effects of anxiety and pain. Sedatives, along with analgesics and other non-pharmaceutical means are regularly utilised to optimise comfort. Sedatives produce a certain degree of anxiolysis, hypnosis and amnesia but most do not have analgesic properties. Shehabi and Innes (2002) define these terms:

- "Anxiolysis refers to the reduction in the emotional and physical response to real or perceived danger and suggests a calm and tranquil state.

- Hypnosis implies a state of minimal motor activity similar to sleep.

- Amnesia or impairment of memory is generally considered desirable, particularly within the intensive care setting" [because of the discomfort and fear that patients experience]. (p.143)

Promotion of patient comfort, while always a goal for nursing care, has a new feature to its meaning. There is a developing body of knowledge around the adverse effects of pain and anxiety, increased scrutiny from accreditation organisations, and growing consumer recognition that the provision of quality care includes optimal comfort (McGaffigan, 2002). It is important to note however that sedatives and analgesic medication are uniquely different agents, having distinct, although complimentary and sometimes synergistic actions.

Studies have documented memory of painful experiences among ICU patients and these experiences have been associated with the development of posttraumatic stress disorder (Fontes Pinto Novaes, Knobel, \& Bork, 1999; Rotondi, Schulz, \& Sirio, 1998). Despite this nursing and medical professionals can overlook pain control and sedation. Unfortunately adequate sedation can become obscured among the myriad of haemodynamic, respiratory and metabolic derangements evident within critically ill patients. Pain and sedation may become an after-thought in very critical patients. The safe and effective application of sedation must be an integral part of intensive care practice. Ostermann, Keenan, Seiferling and Sibbald (2000) suggest that the ideal sedative in the ICU setting 
would have a fast onset of action, be effective at providing adequate sedation, allow for rapid recovery after stopping the drug, be easy to administer, lack drug accumulation, have very few adverse effects, interact minimally with other drugs and be inexpensive. Regrettably such a drug does not yet exist and combinations of drugs are required to meet most of these ideals.

\section{Sedation and the Stress Response}

Inadequate sedation and pain are well known contributors to the stress response producing anxiety and agitation. White, Hollet, Kress and Zellinger (2001) state stress is "a multifactorial behavioural, physiologic, and metabolic cascade in response to traumatic injury, surgery and/or sepsis" (p.6) and is compounded by the necessary treatment a critically ill patient in ICU usually receives (for example, mechanical ventilation and invasive monitoring). Stress, anxiety and agitation are also complicated by the ICU environment, which tends to be characterised by bright lights, loud noises, irregular sleep patterns, unfamiliar faces and a real or perceived danger.

Stress is a product of biochemical cellular changes; stimulation of the peripheral, central and autonomic nervous systems; and the release of humoral factors, such as kinins, leukotrienes and prostaglandins. Stress is also influenced by psychological elements such as a foreign environment or a perceived loss of control over oneself. Initially a patient may act in response to stress by showing evidence of anxiety and/or excitement. This ironically heightens the very response that activated the humoral factors and in-turn increases pain and respiratory dysfunction through the release of catecholamines, glucagons, vasopressin, growth hormone and $\beta$-endorphins. Alongside these changes are alterations in the levels of pituitary hormones, such as adrenal secretion of cortisol and corticotropin, whereas thyroid function tends to be suppressed (Epstein \& Breslow, 1999).

Late patient response to stress is regularly associated with events that occur in the ICU, such as respiratory failure, myocardial infarction and haemorrhage. 
These may result in hypoxia, tachycardia, increased myocardial oxygen consumption, immunosuppression, hypercoagulation, persistent catabolism and hypercapnia (White et al., 2001). Even though stress is thought of as a compensatory mechanism, when considering it in the context of the above, it may be viewed as a maladaptive response that can lead to early and serious complications. For these very reasons appropriate sedation is essential to aid recovery in an ICU.

\section{Risks and Benefits of Sedation}

The optimal level of sedation needed for critically ill patients varies amongst patients and within individual patients over time. At one end of the continuum, very deep sedation may be required for severely head-injured patients with unstable intracranial pressures. At the other end, the goal is an awake, calm patient being weaned from mechanical ventilation. Accordingly, assessment of the degree of sedation should be an important part of patient management in ICU. This assessment is complicated when communication is restricted by ventilation and critical illness.

Given how sedatives and analgesics work it is not surprising that inappropriate administration of sedation has potentially serious consequences. Nursing and medical professionals may at times hold a general reluctance to give sedation, concerned that progress to weaning from mechanical ventilation may be impeded. Nevertheless inadequate sedation may lead to life-threatening agitation, hastening myocardial ischaemia or ventilator dysynchrony. It is also commonly associated with unplanned patient removal of endotracheal tubes, enteral feeding tubes and vascular catheters. This adds significant risk to the patient. Therefore it is a delicate balance that is trying to be achieved in managing the sedative requirements of individual patients. It is my observation that both nursing and medical professionals sometimes have difficulty with the difference between the need for analgesia and the need for sedation. From my nursing practice I have noted some staff commonly believe that by administering 
an analgesic drug, they will also satisfy a patient's sedation requirements or vice versa.

On the other hand, too much sedation may produce prolonged alteration in consciousness. This can lead to hypotension, bradycardia, respiratory depression and an increased period of mechanical ventilation (Kollef et al., 1998) resulting in increased ICU length of stay and increased diagnostic testing (Kress, Pohlman, O'Connor, \& Hall, 2000). Prolonged mechanical ventilation also predisposes a patient to an increased risk of ventilator associated pneumonia (Cook et al., 1998), ventilator associated lung injury (Meade, Cook, Kernerman, \& Bernard, 1997) and critical illness associated neuromuscular abnormalities (De Jonghe et al., 1998). These factors extend the economic burden of sedatives beyond an estimated $\$ 1$ billion annually spent in the United States of America (USA) for the purchase of this group of drugs and represent $10-15 \%$ of all ICU drug expenditures in the USA (Kress et al.).

The difficulty with sedation is the varied and often unpredictable effect of critical illness on drug pharmacodynamics and pharmacokinetics. Most information about the effect of drugs has been extrapolated from healthy persons or patients in a stable period of their chronic disease. In the past, this has led to an inappropriate use of some drugs in acutely ill, unstable patients resulting in adverse clinical outcomes (Lerch \& Park, 1999). Failure to identify these effects may lead to drug accumulation, resulting in prolonged encephalopathy and mechanical ventilation. The drug accumulation may then mask the development of neurologic or intra-abdominal complications. Critically ill patients are a heterogeneous group with different underlying co-morbid conditions, severity of illness, monitoring needs and requirements for life support.

\section{Sedation Management in Intensive Care}

Questions continue to surface over what forms an optimal sedation strategy in ICU. Several studies have compared different types of sedatives, attempting to establish which is more cost effective, or the 'best' for long-term sedation versus 
short-term. A recently published systematic review of these studies (Ostermann et al., 2000) suggested there was a lack of randomised control trials for some sedatives currently in use in ICUs around the world. A large number of trials have been published comparing two common sedatives, midazolam hydrochloride (referred to as midazolam) and propofol in several patient groups, while few studies have been conducted using other sedatives, such as opiates, anaesthetics, isoflurane and other benzodiazepines (of which midazolam is only one). Ostermann et al. found that propofol is as effective as midazolam in providing an expected level of sedation. Once a decision is made to wean patients from their sedation, propofol results in a more rapid time to extubation compared with midazolam. It is not clear, however, if this actually leads to a decrease in total ventilation hours or a shorter ICU stay. Studies reviewed by Ostermann et al. also established that propofol results in more problems with hypotension than does midazolam. The potential clinical significance or risk of this is yet to be determined. In New Zealand propofol is expensive compared with midazolam and in my experience propofol is more widely used for short-term sedation, that is, less than 24 hours. Midazolam on the other hand tends to be used as a continuous infusion for patients requiring sedation for more than 24 hours.

Some sedation strategies favour intermittent intravenous (IV) boluses over a continuous infusion. Bolus sedation generally uses less of a drug, accordingly patients recover more quickly from the effects of the drug and the costs are lower (White et al., 2001). Nonetheless these advantages are offset by the reduced comfort of the patient, which in due course may distract the nurses' attention from other equally important patient care issues. In contrast, continuous IV infusions provide a more constant level of sedation, which avoids 'peaks and valleys', thus providing better comfort for the patient. Continuous IV sedation also frees the nurses' time to address multiple issues. Continuous infusions, however, do accumulate in the body over time and as a result patients often become oversedated. This makes their recovery time longer and potentially their length of stay in ICU is prolonged (Kollef et al., 1998). 
Deep sedation through continuous infusions also results in seriously ill patients being unresponsive for extended periods. This hinders the nurse or doctor's ability to determine and explain changes in the patient's neurological state. That is, whether the changes are a result of the sedative or due to clinical deterioration or a new injury. This may likely result in numerous diagnostic tests, such as Computed Tomography (CT) scans of the brain in which the results often point towards complications of management (such as oversedation) rather than a further diagnosis (Ostermann et al., 2000). A recent study by Kress et al. (2000) has suggested that patients requiring long-term sedation have scheduled sedative interruptions, whereby patients are woken for a brief period of time daily, to allow for assessment of neurological status. The study reported a significant decrease in the duration of stay in the ICU. There was also a reduction in the need for diagnostic tests (e.g. CT scans) that are usually done when patients are slow to awaken. These tests are performed to rule out other neurological reasons for a patient not waking. Further similar studies are required to strengthen the results of Kress et al. because their study was done within a population of medical ICU patients only and cannot be generalised to other ICU populations.

\section{Common Sedatives Used in the Wellington Intensive Care Unit}

Sedation practices vary widely between institutions, partly because of institutional preferences and partly because requirements for sedation vary greatly from patient to patient. Non-pharmacological means (e.g. patient reassurance and positioning comfortably in bed) should always be considered part of any attempt to maintain comfort. No single drug can achieve all the indications for sedation and analgesia in the ICU, therefore a combination of drugs each titrated to specific endpoints is currently recommended as an effective strategy (Kress, Pohlman, \& Hall, 2002).

Early data on sedative use derived from operating theatres and short procedures were the bases on which the use of these drugs was extrapolated to the ICU setting. Yet clinical practice with critically ill patients has revealed a different relationship between the sedative and the patient and the build-up of sedatives in 
these patients remains a significant concern. Critically ill patients may have altered hepatic or renal function which impairs drug clearance. Drug-to-drug interactions, altered protein binding and circulatory instability are common in ICU patients (Kress et al., 2002). In the ICU sedatives typically exhibit a tendency to accumulate in the tissues ensuring a prolonged clinical effect. Midazolam, Propofol, Clonidine, Thiopental Sodium and Opiates are sedatives or have sedative attributes which are used routinely in Wellington ICU, other medications, such as Diazepam, may be used in specific patients; however it is beyond the scope of this paper to discuss them all in detail.

Midazolam is one sedative from the family of benzodiazepines, which also includes Lorazepam and Diazepam. It decreases anxiety and promotes sedation, hypnosis, amnesia, and muscle relaxation. Benzodiazepines stop the acquisition and encoding of new information by the brain, subsequently they block potentially unpleasant experiences without inducing retrograde amnesia (Jacobi et al., 2002). Midazolam is commonly used as preoperative sedation and for light sedation during short diagnostic procedures. Within ICU this drug is usually given intravenously and is generally used to sedate intubated and mechanically ventilated patients.

Midazolam works on many levels of the central nervous system to produce shortterm, generalised central nervous system depression (De Jong \& Karch, 2000). Jacobi et al. (2002) report that although benzodiazepines have no analgesic properties, they have an opioid-sparing effect by tempering the anticipatory pain response. The effects of midazolam are dependent on the dose dispensed, the route of administration, and the use of other drugs in combination. Nevertheless midazolam does have haemodynamic consequences which can adversely effect a critically ill patient. These include; decreasing the mean arterial pressure (MAP), decreasing cardiac output (CO), and decreasing systemic vascular resistance (SVR). Heart rates less than 65 beats per minute also tend to increase slightly whilst heart rates greater than 85 beats per minute tend to slow slightly (De Jong \& Karch). These cardiovascular effects are always taken into account 
when managing a critically ill patient, especially those with shock. Midazolam can have enhanced and prolonged effects if it interacts with barbiturate anaesthetics, tranquilisers and general anaesthetics. Its hypnotic effects are accentuated with the use of narcotic analgesics such as morphine (De Jong \& Karch). In Wellington ICU midazolam and morphine infusions commonly run together in a single syringe. This makes titrating the drugs to the individual patients' specific needs problematic.

In critically ill patients with hepatic and renal impairment the clearance of midazolam can be prolonged. One of the concerns with midazolam is with a continuous infusion, accumulation of the parent drug or its metabolites may result in unintended oversedation. Like many sedatives used in ICU, midazolam tends to accumulate in the adipose tissue of critically ill patients consequently once an infusion is discontinued tissue stores of the drug will then redistribute back into the circulation, for this reason it may take some time for the body to clear the drug completely and the effects to diminish (Kress et al., 2002). Awakening times are therefore unpredictable. It is also documented in the literature that patients who receive a continuous infusion over an extended period of time may go through withdrawal symptoms if the drug is suddenly withheld (De Jong \& Karch, 2000). Therefore it is important patients are weaned from Midazolam in a controlled manner to minimise these effects.

Propofol is a sedative commonly used for the induction and maintenance of anaesthesia in operating theatres. In small doses it can be used for conscious sedation during diagnostic procedures. In Wellington ICU propofol is regularly used as a continuous infusion to sedate patients who are intubated. It is a popular sedative because it has a rapid onset of action and a short period of sedation.

Once stopped propofol allows for a rapid awakening for neurological assessment. If propofol is administered with narcotics, benzodiazepines, barbiturates or other inhalational anaesthetics, then there is an increased 
likelihood of central nervous system depression which may result in a longer time to awakening (Jacobi et al., 2002). Propofol has no analgesic properties and for that reason pain relief must also be given in combination with it. The precise mechanism of action of propofol is unknown. Propofol, like midazolam, has cardiovascular effects. These include; decreasing blood pressure, decreasing $\mathrm{CO}$ and decreasing respiratory rate or apnoea. Propofol tends to have more of an impact on a patient's blood pressure than midazolam if given as a bolus. Propofol is an emulsion in a phospholipid medium and it cannot be used in patients with an allergy to soya bean or eggs (De Jong \& Karch, 2000). High dose or long term infusions may result in hyper-triglycerdemia. Jacobi and colleagues recommend alternative sedatives be considered for critically ill patients with escalating inotropes or vasopressor requirements or cardiac failure.

Clonidine is a central $\alpha$-agonist which is occasionally used in Wellington ICU to enhance the effects of analgesics and general anaesthetics, in order to manage drug withdrawal symptoms. These symptoms may occur after long-term sedation with morphine and midazolam infusions. When attempting to awaken a patient, they may become agitated and at risk of prematurely removing monitoring equipment or their endotracheal tube. Clonidine therefore may be useful in 'smoothing out' the period of withdrawal.

Thiopental sodium (referred to as Thiopentone) is a general barbiturate anaesthetic and anticonvulsant which is used on occasions in Wellington ICU, specifically in cases of severe head trauma. Thiopentone depresses the central nervous system in order to produce hypnosis and anaesthesia with analgesia (De Jong \& Karch, 2000). Thiopentone is neurologically protective and lowers basal metabolic rate, decreases cerebral oedema thereby extending the tolerable ischemic time. Thiopentone may cause hypotension and respiratory depression and its effect can be enhanced in the presence of narcotics.

Opioids are not strictly sedatives, however while their role in ICU is as analgesics, they still produce euphoria, sedation and respiratory depression by 
acting as an agonist at specific opioid receptors in the central nervous system (De Jong \& Karch, 2000). Fentanyl is a short acting analgesic which is also used as an adjunct in the maintenance of general and regional anaesthesia (MIMS, 2003). Interactions with other central nervous system depressant drugs will have additive effects with fentanyl and with morphine.

Despite the diverse goals of sedative therapy in ICU nurses and doctors only use a small number of medications to achieve them. This disparity between the many indications for sedation and the availability of only a few medications (with numerous non-specific effects that can be enhanced in the critically ill patient) may partially explain the complexity of assessing and managing sedation in ICU. Few studies have compared the intensities of different sedation on critically ill patients (Weinert, Chlan, \& Gross, 2001). In the past 20-30 years there has been a concentration on the development of assessment tools that assist health professionals in determining the effectiveness of sedation and a means of assessing more uniformly sedation in ICU patients. Without accurate sedation assessment it becomes difficult to manage the sedatives needs for individual patients. Nurses have a major role to play in this assessment.

\section{Nursing Assessment of Sedation}

Critically ill patients are often scared, disorientated and isolated. They are surrounded by plastic tubes, ventilators, bright lights and strange noises. They have also lost intimate contact with their families or loved ones, are surrounded by strangers and are no longer in control. Providing comfort to these patients continues to be complex, with difficulty evaluating and managing pain, delirium, sedation and anxiety needs in a heterogenous set of patients with variable illness acuity. In the absence of tools, assessing the adequacy of sedation can be problematic because of its largely subjective nature. It is a bedside procedure in which the nurse's participation is vital; he or she will frequently notice changes in the patients' optimal level of sedation because they are present at the bedside continuously. Preferably the goals for a patient would include comfortable, breathing in synchrony with the ventilator; be orientated and able to follow simple 
commands (such as squeezing a hand) without precipitating unnecessary agitation. Unfortunately as the severity of illness intensifies, it can become more difficult to reconcile the goals of assuring a calm, comfortable patient while preventing the accumulation of sedatives.

Titrating sedatives presents a significant challenge to nursing staff at the patients' bedside. Continuously monitoring and assessing patients' level of sedation is complex without a framework on which to base an assessment. It also presents a challenge to medical staff in determining what an appropriate sedative agent for an individual patient is. Until recently, not a lot of attention has been given to thoroughly assessing the effects of sedation on patients, and many ICUs still report little specific consideration around this practice (Botha \& LeBlanc, 2002; Soliman et al., 2001). Botha and LeBlanc recently exemplified this in a national survey of Australian ICUs, looking at sedation practices. Their survey had a response rate of $61 \%$ and found that $43 \%$ of ICUs used sedation-scoring scales and $23 \%$ of units used a sedation protocol. This raises the question how is sedation assessment and management being performed? More significantly complications of sedation had only been audited in $12 \%$ of the ICUs surveyed. To my knowledge this type of survey has never been undertaken in New Zealand.

ICUs internationally rely upon the clinical judgment and experience of their intensive care nursing and medical staff. In the Wellington ICU sedation needs for individual patients are usually discussed on the ward round; the sedatives are then usually prescribed by a physician and administered by the nurse, often with a wide margin of professional discretion in managing sedative therapy. Nurses in the unit have a variety of experience in critical care and are given considerable autonomy in titrating the dosage of sedation. Often physicians' orders prescribed analgesics and sedatives as combination therapy, for example, morphine and midazolam. If there are then any further concerns the medical staff are called back for further discussions with the nurse concerned. 
Nursing assessment of sedation is an assessment that involves both consideration of patient behaviour; response to verbal and physical stimuli, and more objective observations including blood pressure, heart rate, respiratory rate and general appearance. This is often coupled with the nurse's clinical intuition and past experiences. This is supported by Benner and colleagues (1999) who suggest that nurses make qualitative distinctions everyday in their practice and these are often split-second decisions based on differences in colour, temperature, smell, sound, texture, tone, shape and size. In applying this argument to the sedation assessment tools, the difference in scores occurs because in the mind of the rater, the weight of evidence of sedation-agitation on the whole is an aggregate made up of various behavioural characteristics and the weighting attached to each feature. Nurses' and doctors' assessment of sedation may differ in terms of which features of the patient they notice and the credence they associate with each; ultimately this will influence how they manage sedation for the individual patient. From personal experience, undersedation does not often require a stimulus-response assessment because a patient's behaviour indicating undersedation (for example, distressed facial expressions, ventilator dysynchrony, or grasping at the endotracheal tube) tends to occur spontaneously. Notwithstanding this, if the demands of a patient condition are great and changing rapidly, a close assessment of sedation needs may not be done as regularly as would be desired, as a consequence a patient may become deeply sedated (Weinert et al., 2001). In my experience nurses justify the level of sedation used as a rationale for maintaining patient safety, reducing patient stress and keeping them comfortable. Therefore communication between the nurses and doctors is essential, so the goals of care are achieved and both professions clearly understand what each is meaning when describing a patients' behaviour.

It has already been established that contextual factors influence nurses decisionmaking practices in ICUs (Bakken Henry, 1991; Bucknall, 2000). Egerod (2002) demonstrated that the level of nursing skill in ICU was inversely associated with the level of sedation given. Experienced nurses tended to use less sedation than 
less experienced nurses. Experienced nurses have an extensive range of options to work within their practice including early consideration of non-pharmacological means of promoting comfort and reducing agitation. They also tend to incorporate sedation-scoring tools into their practice and use them within the context of individual patient's needs. In Egerod's study there was no delineation of nursing responsibility by rank at the study sites as is also the case in Wellington ICU. All nurses were expected to administer prescribed sedative agents to their patient and use their clinical judgment to determine the rate of infusion or the dose of a bolus to give. Egerod concluded sedation practices are inconsistent, in addition experienced nurses provided better quality sedation management than less experienced nurses in the ICUs she studied. Less experienced nurses tended to over-sedate patients possibly because they spent more time coming to terms with the technology and the extensive knowledge required to work in a critical care environment.

A study by Weinert and colleagues (2001) also considered factors influencing nurses' delivery of sedation. One of their key findings which resonates with my own practice is the influence of nurses' beliefs about and attitudes toward sedation and critical illness. Nurses maintained three main goals of sedative therapy; comfort, amnesia and safety. Nurses commonly use adjectives such as 'comfortable' to describe their patients and this notion, to a nurse, incorporates characteristics of pain control, relief from anxiety, a lessening of respiratory distress and dyspnoea, and a lack of awareness of surroundings (Weinert et al.). Many critical care nurses viewed the lack of awareness or induction of amnesia as desirable for their patients. Even in the Wellington ICU I have overheard nurses using emotive language when discussing amnesia such as 'awful experience', 'they don't need to remember it', 'distressing remembering bad experiences'. Nurses also use sedation to maintain the safety of the patient in minimising the patients' risk of self harm. Behaviours such as climbing out of bed or removing endotracheal (ET) tubes or IV lines are not desirable and put the patient at significant risk. Distressed patients may also bite down on ET tubes and occasionally bite through them. These types of behaviours occur with no 
forewarning and medical staff may not be present, for that reason nurses prefer to have easy access to rapid acting sedatives in order to stabilise patients assessed to be at risk. Interestingly, nurses in Weinert et al. study also reported that the lack of conclusive scientific evidence of the ideal sedation strategy was justification for their use of personal experience to support their practice. Conflict can then arise when individual nurses collaborate with other nurses or medical staff who have different attitudes to sedation or view it as less of a clinical priority.

An unexpected finding from Weinert et al.'s (2001) study was that a patient's family members may influence sedation therapy. Weinert et al. describes such incidences as family members overstimulating their loved one thus the nurse administers extra sedation for comfort. This practice is usually short-lived as the nurse educates the family about appropriate touch and interaction. Before long families also observe that the nurse and doctor equally control sedatives which provide a 'comfortable' state for their loved-one. Nurses may become a focus of influence (through discussions with the nurse) if the family considers the patient is not receiving the full benefits of sedation (Weinert et al.). Whilst I have observed families overstimulating a loved-one in my own ICU, I am not aware of families actively influencing nurses' sedation management in the unit. The degree families are able to influence nurses sedation practices in an ICU is likely to require further study.

\section{The Language of Sedation}

Determining the appropriate sedative for the individual patient is the responsibility of the medical staff and requires a collaborative effort between the nurses and doctors. Clear communication is crucial to ensure there is an understanding between the professionals with regard to what are the concerns of the nurse and the sedative needs of the patient. The language used between nurses and doctors in the ICU context has a significant influence on this process.

A study published by Egerod (2002) attempted to determine how nurses and doctors describe sedation, what contextual factors may influence their decision- 
making practice and whether the level of nursing skill relates to the level of sedation. Egerod's investigation demonstrated that sedation terminology can have an impact on the way staff trouble-shoot problems associated with sedation. For example it was ascertained that the account 'not following the ventilator' may be interpreted as non-compliance and treated with sedation, whereas 'patient ventilator-asynchrony' could be interpreted as a mark of inappropriate ventilator settings. A casual vocabulary for sedation was found at all four sites that were engaged in the study. My experience working in Wellington ICU indicates a similar informal vocabulary amongst nurses and doctors. It is remarkable that an informal language exists internationally for sedation, despite a lack of common definitions. Egerod suggests that one explanation for this terminology is a result of it occurring in the literature. 'Fighting the ventilator' (Tobin \& Fahey, 1994) and 'difficult to wean' (Slutsky, 1994) are common terms in the literature concerning sedation and mechanical ventilation, although they may not have been defined clearly.

Even though the accepted common goal for sedation is to promote patient comfort, breathing and safety, Egerod (2002) found in her study that the only rationale articulated in the ICU was 'tube acceptance'. This nebulous term implies a discrepancy between the proposed and real sedation practices as the term is interpreted differently by various participants in the study. Egerod posits that the ambiguous terminology surrounding sedation may be because medical staff are unwilling to hand over power to nurses whilst at the same time they are reliant on nurses to make salient treatment decisions. This contradiction is addressed by preserving vague terminology which negotiates both positions. Doctors prescribe the sedation and the nurses are granted the autonomy to act on their own decisions. This autonomy is a 'given' (practice tradition) within the critical care culture and is not necessarily written in legislation, protocols or procedures. Egerod recommends sedation terminology is appraised, defined and included in future critical care curriculum. Patients may be time and again inappropriately sedated because of a lack of problem identification, inadequate communication between the professions or blurred professional boundaries. 
From the practice experiences considered in nursing assessment of sedation, there is one key issue which needs to be addressed; the issue of nurseprescribing in ICU. Titrating dosages of sedation is similar to prescribing medication in that it requires of the nurse a comprehensive knowledge of the drug, its potential side effects and interactions with other medications, and its impact on an individual's physiological condition.

\section{Nurse Prescribing in New Zealand}

Nurse prescribing is an issue that has been raised, debated and implemented in various parts of the world. Nurse prescribing and advanced nursing practice are issues the profession has also debated within New Zealand. Although both issues have received attention in the past, amendments made to the Medicines Act (1981) in 1999 and regulations in 2001, have opened the way for independent nurse prescribing by registered Nurse Practitioners ${ }^{\mathrm{TM}}$. The amendments currently only allow nurses practising in the sectors of aged care, sexual and reproductive health, and child family health to prescribe medicines from a specified list. There are likely to be more domains of practice in the future for Nurse Practitioners ${ }^{\mathrm{TM}}$ including mental health, disease management, perioperative, palliative care, emergency and trauma, primary health care and high dependency (Nursing Council of New Zealand, 2002c).

The New Zealand medical profession have argued that nurse prescribing broadened nurses scope of practice. Professionals within nursing suggested that

it only legitimised what was already occurring (Gunn, 1999). Nurse prescribing has come about in New Zealand with the development and credentialing of Nurse Practitioners ${ }^{\mathrm{TM}}$. Nurse Practitioners ${ }^{\mathrm{TM}}$ are registered nurses with at least four years experience working in their chosen clinical area. These nurses are described as expert practitioners.

Nurse prescribing is controlled by the Medicines Act (1981) and the Nursing Council of New Zealand Nurse Practitioner ${ }^{\mathrm{TM}}$ competency requirements. 
Independent prescribing offers Nurse Practitioners ${ }^{\mathrm{TM}}$ the autonomy to make their own prescribing decisions in addition to being accountable for them. Safe nurse prescribing is dependent on the advanced knowledge, skills, expectations, and sound clinical judgment of the nurse prescriber. Nurse prescribers can only prescribe within their specialty area and must be certified Nurse Practitioners ${ }^{\mathrm{TM}}$. Nurse prescribing is tightly regulated and as of June 2003 only one Nurse Practitioner ${ }^{\mathrm{TM}}$ had taken on this extended role. Paula Renouf is a nurse prescriber in child and family health (Prescriptions Come Naturally, 2003). The Nursing Council of New Zealand has very specific regulations controlling nurse prescribing that include requirements for commencing prescribing, ongoing training requirements and competency assessment (Nursing Council of New Zealand, 2002a, 2002b).

In December 2002 the New Zealand Government also released a set of regulations that set out the requirements for standing orders. The purpose of the guidelines was to offer direction for the development and process of standing orders and to support people working under standing orders. A standing order is:

a written instruction issued by a medical practitioner or dentist, in accordance with the regulations, authorising any specified class of persons engaged in the delivery of health services to supply and administer any specified class or description of prescription medicines or controlled drugs to any specified class of persons, in circumstances specified in the instruction, without a prescription. A standing order does not enable up person who is not a medical practitioner or dentists to prescribe medicines-only to supply and/or administer prescription medicines and some controlled drugs (Ministry of Health [MOH], 2002, p.2).

The $\mathrm{MOH}$ regulations require that a standing order lists the medicine to be administered, the indications for which the medicine is to be supplied including the recommended dose or dose range; the contraindications for the medicine, the method of administration and the documentation required $(\mathrm{MOH}, 2002)$. The regulations also note that at this time the Medicines Act (1981) does not cover 
standing orders and a law change is required before standing order regulations can be included within the Act. Accordingly any standing orders for sedative infusions and protocols within ICU are currently not covered by the Medicines Act (1981). It is my opinion that this is often the case in health care whereby advances in practice are made and the law lags behind.

As has been discussed previously sedation prescribed by medical staff in the ICU setting has at times been prescribed with wide margins of discretion for the nurses caring for individual patients to titrate. Nevertheless nurses in critical care environments must be aware of the political/legal context to the practice and ensure they do not administer any medicines including sedation that is not precisely prescribed by a medical practitioner. If nurses titrate sedative infusions and determine the need for an extra bolus dose of sedation, it could be viewed as nurse prescribing in a setting which does not currently hold a mandate in law. Titrating sedation requires a knowledge of the drug, its side effects and potential interactions. This is a task-delegation that critical care nurses have taken on (Bowler \& Mallik, 1998). Often sedation prescribing practices are based upon the tradition and culture of the ICU and nurses work within this practice to provide what they believe to be appropriate administration and/or dispensing of sedation. I believe however, it is unlikely that many critical care units in New Zealand have standing orders (that comply with the new regulations) for sedation practices.

In titrating and administering sedatives nurses require not only an extensive knowledge of the medications but also an understanding of what is happening to the critically ill patient and the impact the medication will have on that patient. Nurses must be able to provide a rationale for the decision they have made and the resulting action. Sedation-scoring tools provide a framework for evaluating the effectiveness of sedation administered to individual patients by guiding the assessment of the patient. 


\section{Sedation-Scoring Tools}

Significant complications of sedation practices in critically ill patients have been established in recent times (Kress et al., 2002) and efforts to alter these practices in ICU have commenced. One such development is the Bispectral Index (BIS). This is an instrument for the measurement of effects of anaesthetics and sedatives on the brain and consciousness. BIS analyses the Electroencephalography (EEG) signal and the changes in this signal over time (Olson, Chioffi, Macy, Meek, \& Cook, 2003). The BIS sensor is not invasive and recent studies (Riker et al., 2000; Simmons et al., 1999) indicate that this monitor works well in some patient groups. The BIS monitoring system is relatively new to critical care; the BIS was originally designed for anaesthetised patients in operating theatres. Whilst it has established validity and reliability in the operating room, within the ICU setting the validity and reliability of the BIS is yet to be fully confirmed. Although the BIS has shown promise as a useful tool for objective sedation assessment in ICUs, questions are still being explored as to what conditions it provides a reliable performance for assessing patient sedation. BIS appears to be a valuable tool in patients requiring deep sedation and neuromuscular blockade, where other sedation-scoring tools are not applicable. There does appear to be some variability with BIS measurement being influenced by the type of sedative and analgesic used (Fraser \& Riker, 2001). BIS is reportedly the only currently commercially available technology of this kind to have some proven clinical application (Riker et al.; Simmons et al.). Additional research continues into the BIS monitoring system to establish its role in the ICU setting.

Other sedation assessment systems include the emergence of sedation-scoring tools. Sedation-scoring tools aim to reduce the subjectivity in sedation assessment by creating objectivity and consistency. Sedation-scoring tools provide descriptors of specific patient behaviour that a number is assigned to. Many sedation-scoring tools have been developed in order to assist health professionals in assessing and managing sedation in critically ill patients. Reliable sedation-scoring tools can improve communication amongst nurses and 
doctors, improve the consistency in drug administration and be used in combination with sedation protocols to improve the precision of sedative titration as patient needs change over time (Sessler et al., 2002).

Evaluating the adequacy of sedation can be difficult because of the subjective component to assessing patient behaviour. Sedation-scoring instruments usually include descriptions of some of the following: agitation, pain, consciousness, haemodynamic variables, anxiety, synchrony with the ventilator and reaction to tracheal suctioning. Not all assess both agitation and sedation. Some sedationscoring tools view sedation and agitation as part of the same concept at divergent ends of a continuum, for example the Sedation-Agitation Scale (SAS) (Table 1). Whereas other scoring tools treat these as two separate concepts completely, for example the Ramsay Scale (see also Table 1). In some cases agitation is not considered as part of a sedation-scoring tool. Even though the sedation scales available are different in design and use, they require the assessment of the patient's response to some form of stimulus. The assessment may be based on the patient's response to light or deep touch (which usually involves a trapezus muscle squeeze or pressure on the nail beds, someone calling their name; a loud noise, such as a clapping sound; or someone giving them a verbal command, such as asking the patient to squeeze your hand or open their eyes). The patient's response to the stimulus is assessed in accordance with a scoring system. Most sedation-scoring tools have a numerical value allocated to clinical findings. A change in the score over a period of time is used to evaluate the effectiveness of the sedation strategy to a predetermined goal. The aim of these scoring tools is to assess the level of sedation so sedatives can be titrated enabling patients to be comfortable, co-operative, and compliant with their care. These tools may, however, be prone to poor inter-rater reliability, variability and bias (McGaffigan, 2002). 


\title{
Table 1. Subjective Scales to Rate Sedation and Agitation
}

\author{
Ramsay Scale \\ 1- Anxious and agitated or restless or both \\ 4- Brisk response to light glabellar tap \\ 2- Co-operative, oriented and tranquil \\ 3- Responding to commands only \\ 5- Sluggish response to light glabellar tap \\ 6- No response to light glabellar tap

\section{Sedation - Agitation Scale (SAS)} \\ 7- Dangerous agitation. Pulling at ET tube, trying to remove catheters, climbing over bed rail, \\ striking at staff, thrashing side-to-side. \\ 6- Very agitated. Does not calm, despite frequent verbal reminding of limits; requires physical \\ restraints, biting ET tube. \\ 5- Agitated. Anxious or mildly agitated, attempting to sit up, calms down to verbal instructions. \\ 4- Calm and co-operative. Calm, awakens easily, follows commands. \\ 3- Sedated. Difficult to arouse, awakens to verbal stimuli or gentle shaking but drifts off again, \\ follows simple commands. \\ 2- Very sedated. Arouses to physical stimuli but does not communicate or follow commands, may \\ move spontaneously.
}

1- Unrousable. Minimal or no response to noxious stimuli, does not communicate or follow commands

\section{Motor Activity Assessment Scale (MAAS)}

0- Unresponsive. Does not move with noxious stimulus.

1- Responsive only to noxious stimuli. Open eyes OR raises eyebrows OR turns head toward stimulus OR moves limb with noxious stimulus.

2- Responsive to touch or name. Open eyes OR raises eyebrows OR turns head toward stimulus OR moves limb when touched or name is loudly spoken.

3- Calm and co-operative. No external stimulus is required to elicit movement AND patient is adjusting sheets or clothes purposefully and follows command.

4- Restless and co-operative. No external stimulus is required to elicit movement AND patient is picking at sheets or tubes OR uncovering self and follows command.

5- Agitated. No external stimulus is required to elicit movement AND attempting to sit up OR moves limbs out of bed AND does not consistently follow commands (e.g. will lie down when asked but soon reverts back to attempts to sit up or move limbs out of bed).

6- Dangerously agitated, unco-operative. No external stimulus is required to elicit movement AND patient is pulling at tubes or catheters OR thrashing side-to-side OR striking at staff OR trying to climb out of bed AND does not calm down when asked. 
In 1974, the first description of a sedation-scoring instrument was published by Ramsay, Savege, Simpson, and Goodwin (1974). The Ramsay Scale is still used by many ICUs through out the world today. This simple tool has only a few general descriptors with six levels of scoring. Table 1 provides a comparison of the Ramsay scale along with two more recently developed sedation-scoring tools including the SAS and the Motor Activity Assessment Scale (MAAS), which was derived from the SAS (Devlin et al., 1999). The Ramsay scale has been criticised because it offers only one level for agitation and the descriptors lack detail (McGaffigan, 2002). In contrast, the SAS has seven levels that offer three levels each for advancing sedation and agitation, with one level for a calm and cooperative patient. There are explicit behavioural descriptors at each level of both the SAS and the MAAS. There are now over 25 sedation-scoring tools that have been developed and refined in use.

In 2000, De Jonghe et al. published a systematic review of sedation-scoring systems in use internationally. Their study found it difficult to draw any strong conclusions regarding the best tool to employ because many scoring tools had not been independently tested for validity. What De Jonghe et al. did find was that some tools, namely the Ramsay Scale (Ramsay et al., 1974), the Glasgow Coma Scale modified by Cook and Palma (1989), the SAS (Riker et al., 1994), and the MAAS (Devlin et al., 1999), had been coupled and validated against each other. In the analysis completed by De Jonghe and colleagues the correlations between these tools were high and the authors concluded the tools appeared to measure the same constructs. De Jonghe et al. concluded that the high correlations observed between these distinct instruments provided some measure of validation. Details of these correlations are presented in Table 3 of the following chapter. The authors also point out that while these instruments provide an adequate measure of sedation at one point in time, none of the instruments evaluated have been assessed for their ability to detect alterations in sedation level over time within the same patient. On the whole, no sedationscoring system has been evaluated so comprehensively that it would be considered ideal or the 'gold standard', whilst there are numerous in use 
internationally; many lack formal validation (De Jonghe et al.). Despite this ICU staff appear to value these tools and have adopted them readily into practice. People continue to work on the development of sedation-scoring tools and refine them therefore they must have some value in the clinical setting.

\section{Sedation-Agitation Scale}

The SAS was first developed in 1994 as part of a study to assess relieving agitation in critically ill patients with Haloperidol. Riker et al. (1994) developed the SAS to be able to quantify and compare the degree of agitation and sedation experienced by their participating patients. Objectively assessing patients and quantifying sedation and agitation is fraught with difficulty. Riker et al. found that the development of the SAS allowed them to more easily quantify the level of sedation and agitation and to identify the sedation goal for individual patients. The nursing reports of agitation and sedation documented in medical notes were transformed into behavioural descriptors for the various levels of the SAS.

The SAS was originally a 7-point scale from -3 to +3 (Table 2) but once adapted it became a scale from 1 to 7 (Table 3). Expertise from medicine, nursing and pharmacology was then used to modify the tool in 1999. These professionals included one physician and eight experienced ICU nurses. Rogers (2003) suggests that such reinvention is a normal part of the diffusion-innovation process. It allows for the customisation of the innovation (in this case the SAS) to fit more appropriately to local conditions. According to Rogers, diffusion of innovation has within it a notion of clinical testing. In health care many innovations are introduced without clinical testing and often the clinical testing happens at a later stage. This is sometimes known as reinvention. It is often not until this reinvention and adoption into practice is complete that further research is done to affirm the value of the innovation in the clinical setting. 
Table 2. The Original Sedation-Agitation Scale of 1994

\begin{tabular}{|c|c|c|}
\hline+3 & Immediate threat to safety & $\begin{array}{l}\text { Pulling at endotracheal tube or catheters, trying to } \\
\text { climb over bedrail, striking at staff }\end{array}$ \\
\hline+2 & Dangerously agitated & $\begin{array}{l}\text { Requiring physical restraints and frequent verbal } \\
\text { reminding of limits, biting endotracheal tube, } \\
\text { thrashing side-to-side }\end{array}$ \\
\hline+1 & Agitated & $\begin{array}{l}\text { Physically agitated, attempting to sit up, calms down } \\
\text { to verbal instructions }\end{array}$ \\
\hline 0 & Calm and co-operative & Calm, arousable, follows commands \\
\hline-1 & Oversedated & $\begin{array}{l}\text { Difficult to arouse or unable to attend to conversation } \\
\text { or commands }\end{array}$ \\
\hline-2 & Very oversedated & Awakens to noxious stimuli only \\
\hline-3 & Unrousable & Does not awaken to any stimuli \\
\hline
\end{tabular}

Table 3. The Modified Sedation-Agitation Scale of 1999

\begin{tabular}{|c|c|c|}
\hline 7 & Dangerous agitation & $\begin{array}{l}\text { Pulling at endotracheal tube or catheters, trying to } \\
\text { remove catheters, climbing over bedrail, striking at } \\
\text { staff, thrashing side-to-side }\end{array}$ \\
\hline 6 & Very agitated & $\begin{array}{l}\text { Does not calm, despite frequent verbal reminding of } \\
\text { limits; requires physical restraints, biting } \\
\text { endotracheal tube }\end{array}$ \\
\hline 5 & Agitated & $\begin{array}{l}\text { Anxious or mildly agitated, attempting to sit up, } \\
\text { calms down to verbal instructions }\end{array}$ \\
\hline 4 & Calm and co-operative & Calm, awakens easily, follows commands \\
\hline 3 & Sedated & $\begin{array}{l}\text { Difficult to arouse, awakens to verbal stimuli or } \\
\text { gentle shaking, but drifts off again, follows simple } \\
\text { commands }\end{array}$ \\
\hline 2 & Very sedated & $\begin{array}{l}\text { Arouses to physical stimuli, but does not } \\
\text { communicate or follow commands, may move } \\
\text { spontaneously }\end{array}$ \\
\hline 1 & Unrousable & $\begin{array}{l}\text { Minimal or no response to noxious stimuli, does not } \\
\text { communicate or follow commands }\end{array}$ \\
\hline
\end{tabular}

The changes made in 1999 can be seen in Table 3 which is re-presented to allow comparison. These changes included renaming several categories and moving or adding behavioural descriptors. "Immediate threat to safety" was changed to "dangerous agitation"; "dangerously agitated" was changed to "severe agitation"; "oversedated" was changed to "sedated" and "very oversedated" was changed to "very sedated" (Riker et al., 1994). The authors also altered some of the behavioural descriptors. "Thrashing side to side" moved from the level of "severe 
agitation" to "dangerous agitation"; "follows simple commands" was added to the level of "sedated" and "does not communicate or follow commands" was added to "very sedated". Following the theory of Rogers (2003) once the SAS is adapted it is more likely to be compatible with the ICU culture and is therefore likely to be adopted in practice.

The modified SAS has three categories each for sedation and agitation behaviour with one level for calm and co-operative. Example descriptors of patient behaviour are provided at each level to guide the assessor. If a patient is already awake or awakens easily to voice (namely, responds with voice or head shaking to a question or follow commands), the patient would be classified as a SAS of 4 (calm and appropriate). If it takes more stimuli such as a loud voice or shaking but the patient does eventually awaken and responds with voice or head shaking or follows a command that is a SAS of 3 . If the patient arouses to more physical stimuli (may be noxious, such as pain or discomfort) but never awakens to the point of responding to yes or no or following commands, the patient would be assessed as a SAS of 2 . If a patient has little or no response to any stimuli that is a SAS of 1 (R.R. Riker, personal communication, April 16th, 2003). The levels differentiate between those patients that the assessor can eventually wake up (SAS 3), those one cannot awaken but can arouse (SAS 2), and those one cannot even arouse (SAS 1). For agitation if the patient is a little anxious, restless or even mildly agitated but calms down easily, they would be classified as a SAS 5. Despite frequent reminding, if a patient is more persistently agitated, for example biting on the endotracheal tube that is a SAS 6. If the patient is a danger to themselves or the staff, that patient is classified as a SAS 7 (R.R. Riker, personal communication, April 16th, 2003).

Rating sedation using scoring tools can be complex. An issue with rating that Riker et al. (1999) identified from their study with the SAS tool, was that a small number of patients might fall into a 'crossover' situation. That is, they may appear sedated or be difficult to arouse but when stimulated they become significantly agitated. Riker et al. recommend that this should be accommodated by 
assessing patients' behaviour when stimulated because it most likely represents their response to therapeutic interventions, such as physiotherapy, suctioning and repositioning. Riker et al. also provided some guidelines for raters by suggesting that the presence of hand restraints would not mandate a SAS score of 6 , unless the patient's behaviour is as described. Some nurses put hand restraints on prophylactically to minimise the risk of harm to the patient and maintain their safety.

\section{Conclusion}

The goals of sedation in ICU are great and varied. They include physiological, ventilatory, psychological and humanitarian goals that are individualised for each patient. However considerable complications of sedation practices in critically ill patients have been recognised in recent years and efforts to modify these practices have commenced. The difficulty with sedation is the varied and often unpredictable effect of critical illness on drug pharmacodynamics and pharmacokinetics. One of the difficulties with managing sedation in ICU is that nurses have to use their clinical judgment accurately to balance the need for sedation against patient's haemodynamics and respiratory conditions, medical plans, and the desires of the patients and their families.

The goal of sedation can be viewed as a continuum; very deep sedation may be required for specific patients, for example severely head-injured patients with unstable intracranial pressures. At the other end, the goal is an awake, calm patient being weaned from mechanical ventilation. Accordingly, assessment of the degree of sedation should be an important part of patient management in ICU. Nurses spend a considerable amount of time at their patient's bedside continuously monitoring their need for sedation, evaluating their progress and intervening in a timely manner to maintain stability. Sedation assessment can be 'buried' amongst the myriad of other assessments the nurse undertakes in their daily care. The emergence of sedation-scoring tools has provided nurses and doctors with the means to assess their patient's sedation state regularly and 
consistently, and then make the appropriate clinical decision in managing their sedation.

The difficulty with many sedation-scoring tools in clinical use today is that very few have undertaken formal reliability and validity testing. The ideal sedationscoring tool would be economical, easy to use, and highly reproducible, free from inter-rater inconsistencies, and validated in various populations with respect to age, seriousness of illness, specialty units, and the presence of neuromuscular blocking agents. A reliable sedation-scoring tool can improve communication amongst nurses and doctors, improve the consistency in drug administration and be used in combination with sedation protocols to improve the precision of sedative titration as patient needs change over time accordingly minimising the harmful effects of over sedation or under sedation to individual patients.

The SAS adopted into clinical practice by Wellington ICU has had some reliability and validity testing undertaken. One significant flaw inherent in any investigation of a sedation-scoring tool is the non-existence of a true reference measure or criterion for sedation. No such measure exists. No sedation-scoring system has been evaluated so comprehensively that it could be considered ideal or the 'gold standard' and further testing is required to establish its reliability in a heterogeneous set of patients. Despite the lack of formal validation ICU staff around the world value sedation-scoring tools and have adopted them readily into practice. There clearly is an appropriate use of these tools in practice as people continue to work on the development of sedation-scoring tools and refine them for clinical use. The following chapter details the previously published literature on the SAS and identifies the gaps for further research. 


\section{Chapter 4- The Sedation-Agitation Scale}

Most critically ill patients require sedation and analgesia for at least part of their stay in an Intensive Care Unit (ICU). Sedation can reduce agitation, promote synchronised breathing with the ventilator, and assist to relieve anxiety and discomfort associated with the high technologic environment of the ICU. Despite the frequent use of sedation in intensive care, management of sedation in critically ill patients remains one of the more challenging and contentious practice areas in the ICU today. Sedation-scoring tools have been developed as a key assessment strategy to help determine the level of sedation in patients. For a tool to be considered dependable it must be reliable and valid. Whilst there are many tools in use in clinical practice today, very few have received any formal testing of validity and reliability. One tool that has some testing is the Sedation-Agitation Scale (SAS) (Appendix A). This tool has been adopted by Wellington ICU as a strategy for determining the level of sedation in patients in order to prevent over sedation and to minimise the risks associated with under sedation and agitation.

This chapter explores and critiques the literature on the reliability and validity of the SAS. There are two principal studies which have previously evaluated the reliability and validity of the SAS; Riker et al. (1999) and Brandl et al. (2001). This chapter describes the studies through an analysis of their designs, the SAS raters, number of raters, and the setting. This chapter also identifies areas for further research consideration.

\section{The Sedation-Agitation Scale Raters}

Raters in studies of the SAS have been found to differ professionally, by their ICU practice experience, their experience with the SAS tool and by familiarity with the patient. Riker et al. (1999) used trained evaluators, eight ICU nurses and one physician investigator in their reliability study. The trained 'experienced' ICU nurses (mean experience of 5.9 years in the authors' ICU) and the physician worked in pairs to rate patients using the SAS, the Ramsay scale and the Harris Scale. The training of the raters involved three-one hour tutorials and observing 
patients at the bedside as a group then discussing disagreements to develop a consensus. What is not clear from the study is whether this training was done in a separate ICU from the unit that became part of the study and the length of time for the bedside training and classification of patients. These trained investigators were also the same clinicians who participated in modifying the SAS scale (Riker et al).

Riker et al. (1999) defined experience as the number of years worked in their ICU (34-bed medical-surgical ICU) and at the same time associate this with training on the instrument. However no reference is made to the overall level of professional experience of these nurses or the physician. In comparison the reliability study of the SAS published in 2001 by Brandl et al. identifies both the length of ICU experience and the professional level of experience of the participants. Raters included a certified critical care clinical nurse specialist, critical care pharmacist and a pharmacy doctoral student. In the New Zealand context it is not useful to define experience in the same way as Riker et al. as ICUs in this country have a workforce that is more transitory in nature and it is unlikely that many nurses spend 5-8 years in one particular ICU. From my experience New Zealand has a considerable number of international nurses practicing in its ICUs and as a result staff do not stay in one unit for a substantial period of time. Moreover a study by Rowley and Fielding (1991) using the Glasgow Coma Score noted that trained and experienced users of the assessment tool had improved reliability and accuracy compared with trained but inexperienced assessors, furthermore a much better reliability than untrained, inexperienced observers. Whilst Rowley and Fieldings' study provides support for the assertion for training in using the SAS, Riker et al. did not consider the effects less experienced staff would have on the reliability of the SAS.

Brandl et al.'s study (2001) supported the reliability of the SAS by evaluating it with ICU nurses who had no experience with the tool. This appears to be the first published evaluation of the SAS outside of the institution where it was developed. 
Brandl et al. found that the SAS was reliable when administered by staff nurses with no experience in using it. In their study, 27 staff nurses who had not used the SAS before, assessed patients alongside one of two trained investigators (critical care pharmacist and a pharmacy doctoral student) and a critical care nurse specialist familiar with the tool. All staff nurses working in the medical ICU were eligible to participate. The level of professional experience of these staff nurses was not clearly described only that the staff were not familiar in practice with the SAS. The inter-rater agreement between the trained investigators and the ICU staff nurses was excellent ( $\kappa=0.85$ and $0.87, p<0.001$ for both). Brandl et al. did conclude, however, that experienced/trained users of the SAS have better reliability than untrained/inexperienced nurses, as supported by Riker et al. $(\kappa=0.92, p<0.001)$, and recommended that orientation to any subjective assessment tool is important. The fact that nurses in the Brandl et al. study were familiar with assessing sedation in critically ill patients using the Ramsay Scale may have also had an impact on the reliability of the study. In contrast with Riker et al. (1999) who used a physician, Brandl et al. used two pharmacists as their experienced investigators. This introduces the question of whether there is reliability between disciplines using the SAS. Within the New Zealand context it is more likely that a doctor and nurse will be using the SAS therefore it is important to determine the reliability of the SAS when used by these professionals.

The evaluators in the inter-rater reliability study of the SAS (Riker et al., 1999) were not able to look at the patient's progress data sheet or have been caring for the patient during the shift of the ratings. Moreover it is not clear that the raters did not have any prior knowledge of these patients, namely that they had not been present at nursing or medical handover times. Riker et al. argue that by eliminating the primary nurse as one of the raters prevents bias from uneven exposure to the patient. They also suggest that the frequency of behavioural events and other information about the patient, in the natural ICU setting, may influence how the nurse rates the patient. If the primary nurse is not one of the raters, then the raters may be unaware that the primary nurse has just spent the past half-hour providing limit-setting reminders to the patient or for example, has 
given some anti-seizure medication that has made the patient sleepy. In contrast Brandl et al. (2001) did use the primary nurse caring for the individual patient as the inexperienced assessor of the SAS, yet they still found that there was interrater agreement between the staff nurses and the trained investigators; nevertheless it was less than that of Riker et al.

Despite the Riker et al. (1999) study having a mix of raters they did not give any consideration to what impact this may have in any way on the study or whether a nurse and a doctor would give a similar rating on the same patient. It would appear important to consider these factors in future reliability studies given what is known about their different role. Nurses and doctors may each in turn influence how the ratings are performed, which may influence clinical decisions that are made based on these assessments. There appears little locatable published material that considers the difference or similarities between clinical decisions made by nurses and doctors. One study completed in an emergency department (Sakr et al., 1999) compared the care and outcome of patients with minor injuries that were managed by a nurse practitioner or a junior doctor. In this study there was no significant difference between nurse practitioners and junior doctors in the accuracy of examination and treatment, planned follow-up, or requests for radiography. As nurse practitioner roles develop within New Zealand, we may see more studies comparing their outcomes with those of doctors as the roles evolve and the tension between these positions is heightened.

\section{Number of Ratings and Setting}

The setting for the reliability testing that has occurred has been in different ICUs but with similar numbers of patients and ratings. Forty five patients were consecutively assessed 69 times by pairs of raters in Riker and colleagues' (1999) study. Fourteen of these patients were evaluated more than once; two patients were assessed six and four times respectively, four patients were each assessed three times and eight patients were observed twice. The time period over which these evaluations took place is not made explicit. In comparison, 
Brandl et al. (2001) evaluated 60 patients only once per day and they could not then be assessed again on the same day in order to lessen any disturbance to the patient.

In not using the primary nurse as one of the evaluators, Riker et al. (1999) were attempting to ensure there was no familiarity bias in the study. However, it would appear that it was introduced in another fashion. The independent raters of the SAS had different amounts of exposure to the scoring tools as is indicated by the range of ratings (4-29). One rater had done the assessment 29 times whilst another had only done the ratings four times, this range of familiarity with the tools may have had some influence on the study ratings.

The Riker et al. (1999) study was based in a 34-bed medical-surgical ICU, whilst Brandl and colleagues' (2001) study was based in a 14-bed medical-cardiac ICU. As previously stated the Brandl et al. study was the first published reliability study of the SAS outside of the institution where it was developed. The study by Riker and colleagues was not performed in a natural setting. The tool was not already in use in the ICU and was introduced solely for the purpose of their research. Although these are the only two published studies substantiating the reliability of the SAS, it has purportedly already been adopted by other ICUs internationally which may mean that the SAS is viewed clinically as an appropriate tool for assessing sedation and agitation. I personally know that one other ICU within New Zealand and at least one ICU in Australia also use the SAS. Although Riker et al. used a cohort of ICU patients, published evidence of the reliability of the SAS on different groups of ICU patients is yet to be found, for example those with head injuries, specialist surgery and renal patients.

In Riker et al.'s study (1999) some patients were excluded. This included those patients receiving neuromuscular blockade because of the lack of movement and observable behaviour. Brandl et al. (2001) excluded patients with or suspected of underlying neurological deficit, deafness, non-English/non-Spanish speaking and 
those with a life expectancy of less than 24-hours. Even though Riker et al. raises, in their discussion, the issue of assessing patients with a neurological deficit, it is not clear whether they were excluded from the study. Bearing in mind the behavioural descriptors which guide the SAS, the question of whether the SAS or any sedation-scoring tool will be reliable for patients with a neurological deficit is yet to be answered. The ability of a sedation-scoring tool to be reliable in this group of patients may depend on the tool used as each measures different attributes that represent sedation and/or agitation. The exclusion criteria of language is also pertinent to the New Zealand context as in Wellington we have several 'ethnic' communities and my personal experience in the ICU is that we do receive patients not able to speak English. The exclusion criteria discussed by both Brandl and colleagues and Riker et al. highlights the complexity of one tools' ability to quantify sedation and agitation in all patient diagnostic populations.

Given that the level of consciousness and sedation in individual ICU patients fluctuates over time, the capability of a tool to identify change in a patient's clinical condition is a desired measurement characteristic (Guyatt, Walter, \& Norman, 1987). The responsiveness of a sedation-scoring scale to sedative instigation, adjustment in drug dosage, and discontinuation of sedation, is consequently an important property that the evaluative tool should exhibit. De Jonghe et al. (2000) define responsiveness as "the extent to which an instrument can detect important changes in sedation, even if those changes are small" (p.277). In their systematic review they found no sedation-scoring tool that had tested responsiveness. There is still considerable work to be done in considering this important issue with sedation in clinical practice. Although this has not been empirically tested, it would appear that the SAS along with other sedation-scoring tools has some ability to determine responsiveness; otherwise ICUs would not continue to use these tools throughout the world and base clinical decisions on them. In 2001 Riker, Simmons, Fraser and Wilkins published a study attempting to validate the SAS with the Bispectral Index (BIS). This study was performed on cardiac patients waking from surgery and is the first published study indicating that the SAS may be able to detect responsiveness over time. 


\section{Selection of People Rated}

In the Riker et al. (1999) study, patients were not randomised. Potential patients were identified by the evaluators at all ranges of the SAS scale. At the end of each week if it was observed that one level of the SAS was under-represented then that level would be preferentially recruited. This was important for first time reliability evaluation because it was testing the reliability of the instrument by critical care staff and the reliability of all levels of the scale needed to be evaluated. Patients were then assessed to ensure procedures had not been performed or sedating medications given within the previous 10 minutes before assessment could take place. Despite this sampling technique Riker et al. produced data that they argue is representative of the population of the ICU in which the study took place. It is difficult to determine if this is so as limited demographic detail of the ICU is presented. A useful way of presenting information about the type of ICU would have been to present data on the average length of stay, ventilation hours, bed occupancy rates, mortality rates and the Acute Physiological, Age, Chronic Health Evaluation Scores (APACHE II). The APACHE II provides an indication of baseline severity of illness data and is a predictor of hospital mortality risk for critically ill adults (Knaus et al., 1991).

\section{Validity of the Sedation-Agitation Scale}

Validity measures "how well a test actually measures what it proposes to measure by comparison with a gold standard or criterion" (Fraser \& Riker, 2001, p.968). If a standard (or criterion) exists then the new test should be compared with it. While a 'gold standard' may be easily defined for some things it is much more elusive when evaluating tools for observed behaviour (Streiner \& Norman, 1995). There is currently no gold standard against which to evaluate sedationscoring tools therefore other forms of validity testing are necessary.

The terms face validity and content validity are technical descriptors of a judgment that a particular scale appears appropriate. Face validity indicates whether the sedation-scoring tool appears to be appraising what it is supposed to be (Streiner \& Norman, 1995). In comparison content validity comprises of a 
considered opinion of whether a sedation-scoring tool encompasses all the relevant content domains (Streiner \& Norman). These forms of validity comprise of a judgment by experts as to whether a scale seems suitable for its proposed purpose. Riker et al. (1999) used the expertise of experienced ICU nurses to determine the face validity of the SAS. In determining whether a particular sedation-scoring tool is appropriate for an individual unit, such as in Wellington ICU, judgments of face validity were made. Based on the judgments of appropriateness and usefulness the SAS was chosen to be adopted, rather than developing or adopting another tool. However, validity requires more than peer judgments to determine whether a sedation-score measures what it claims to measure. Empirical evidence must also be provided (Streiner \& Norman).

In this situation, where other sedation-scoring tools exist a new instrument can be measured against existing instruments to determine whether there is a strong correlation between the two instruments. This is known as construct validity (Streiner \& Norman, 1995). Construct validity involves "comparisons between measures, and examination of the logical relationship that should exist between a measure and characteristics of patients and patient groups" (De Jonghe et al., 2000, p.277). For example in measuring agitation, it could be compared with the number of times a patient removes a device or the number of times nurses have to calm patients down. In the case of the SAS construct validity was determined by Riker et al. (1999) in comparing it with other instruments (the Ramsay and the Harris Scales) also designed to assess sedation. This form of construct validity testing has been undertaken throughout the evolution of sedation-scoring tools in ICU settings however this is not to say that the other sedation-scoring tools used in comparisons have ideal validity. Some appear to acquire criterion status with little testing. The complexity of the process in testing construct validity arises not from a single powerful experiment, but from a series of converging experiments, involving several studies and several different approaches. The SAS is currently in the process of having its construct validity determined (Brandl et al., 2001; De Jonghe et al., 2000; Riker et al., 1999; Riker et al., 1994; Simmons et al., 1999). 
Of the 25 instruments examined by De Jonghe et al. (2000) in a systematic review of sedation-scoring systems, it was considered difficult to draw any strong conclusions because most scoring tools had not been independently tested for validity. De Jonghe et al. did find that the validity of four tools in particular, namely the Ramsay Scale (Ramsay et al., 1974), Glasgow Coma Scale modified by Cook and Palma (1989), SAS (Riker et al., 1999), and the Motor Activity Assessment Scale (MAAS) (Devlin et al., 1999) had validity determined to some degree. Table 4 is modified from an extensive table presented by De Jonghe et al. to show the comparison of the four scales that have undergone some formal methodological evaluation. The Ramsay Scale has been validated against the Glasgow Coma Scale modified by Cook and Palma ( $r=0.89-0.92$ on 4 different occasions). The SAS has been validated against the Ramsay Scale $(r=0.91$, $p<0.001)$ and the Harris Scale $(r=0.93, p<0.001)$. The MAAS had been validated with a Visual Analogue Scale (VAS) $(\kappa=0.83, p<0.001)$. All resulted in high positive correlations, supporting the SAS construct validity. These scales have a similar structure and subject matter which may perhaps explain some of the high correlations. The measure of this validity, however, is limited by the degree to which the content and structure of the scales being compared are similar. It is of note that each of the reference instruments used in these studies had also not been independently evaluated for validity. The studies each used various types of ICU patients and not one sedation-scoring tool has been evaluated on the broad spectrum of ICU patients. The validity of the SAS has also been confirmed in a limited way with the BIS in one particular group of ICU patients, namely cardiothoracic patients awakening after surgery $(r=0.60, p<0.001)$ (Riker et al., 2001; Simmons et al., 1999). 
Table 4. Methodological Evaluation of Sedation-Scoring Tools

\begin{tabular}{|c|c|c|c|c|c|c|}
\hline \multirow{2}{*}{\multicolumn{2}{|c|}{ Name }} & \multirow{2}{*}{$\begin{array}{l}\text { Validation } \\
\text { Study }\end{array}$} & \multirow[t]{2}{*}{ Population } & \multicolumn{3}{|c|}{ Validation Process } \\
\hline & & & & $\begin{array}{l}\text { No. of } \\
\text { assessments }\end{array}$ & Reliability & Validity \\
\hline \multirow{2}{*}{\multicolumn{2}{|c|}{$\begin{array}{l}\text { Ramsay } \\
\text { scale }\end{array}$}} & $\begin{array}{l}\text { Carrasco } \\
(1993)\end{array}$ & $\begin{array}{l}102 \text { adult } \\
\text { patients }\end{array}$ & $\begin{array}{l}1040 \\
\text { measurements } \\
\text { (\# of raters } \\
\text { unclear) }\end{array}$ & & $\begin{array}{l}\text { Validity vs. GCS modified } \\
\text { by Cook \& Palma: } r=0.89 \\
\text { to } 0.92 \text { on four different } \\
\text { occasions, } p \text { value NR }\end{array}$ \\
\hline & & $\begin{array}{l}\text { Riker } \\
(1999)\end{array}$ & $\begin{array}{l}45 \text { adult } \\
\text { patients }\end{array}$ & $\begin{array}{l}69 \\
\text { assessments } \\
\text { by pairs of } \\
\text { raters }\end{array}$ & $\begin{array}{l}r=0.87, p<0.001 \\
\text { Weighted kappa } \\
0.79, p<0.001 \\
\text { Weighted kappa } \\
0.79, p<0.001\end{array}$ & $\begin{array}{l}\text { Validity vs. SAS: } r=0.91 \\
p<0.001\end{array}$ \\
\hline \multicolumn{2}{|c|}{$\begin{array}{l}\text { Sedation } \\
\text { Agitation } \\
\text { Scale (SAS) }\end{array}$} & $\begin{array}{l}\text { Riker } \\
(1999)\end{array}$ & $\begin{array}{l}45 \text { adult } \\
\text { patients }\end{array}$ & $\begin{array}{l}69 \\
\text { assessments } \\
\text { by two raters }\end{array}$ & $\begin{array}{l}r=0.91, p<0.001 \\
\text { Weighted kappa } \\
0.92, p<0.001\end{array}$ & $\begin{array}{l}\text { Validity vs. Ramsay scale: } \\
r=0.91, p<0.001 \\
\text { validity vs. Harris scale: } \\
r=0.93, p<0.001\end{array}$ \\
\hline \multicolumn{2}{|c|}{$\begin{array}{l}\text { Motor } \\
\text { Activity } \\
\text { Assessment } \\
\text { Scale } \\
\text { (MAAS) }\end{array}$} & $\begin{array}{l}\text { Devlin } \\
\text { (1999) }\end{array}$ & $\begin{array}{l}25 \text { adult } \\
\text { surgical } \\
\text { patients }\end{array}$ & $\begin{array}{l}400 \\
\text { assessments } \\
\text { by two raters }\end{array}$ & $\begin{array}{l}\text { Kappa } 0.83(95 \% \\
\text { Cl 0.72-0.94) }\end{array}$ & $\begin{array}{l}\text { Validity vs. expert VAS } \\
\text { assessment: GEE } p< \\
0.001, \text { Validity vs. change } \\
\text { in HR: GEE } p<0.001 \text {, } \\
\text { Validity vs. change in BP: } \\
\text { GEE } p<0.001 \text {, Validity } \\
\text { vs. recent occurrence of } \\
\text { agitation-related events: } \\
\text { GEE } p<0.001\end{array}$ \\
\hline \multicolumn{4}{|l|}{ Pts: } & \multicolumn{3}{|c|}{ VAS: Visual analogue scale } \\
\hline \multicolumn{4}{|c|}{ \#: $\quad$ Number } & \multicolumn{3}{|c|}{ GEE: Generalised estimating equation approach to regression } \\
\hline GCS: & \multicolumn{3}{|c|}{ Glasgow Coma Scale } & \multicolumn{3}{|c|}{ HR: Heart rate } \\
\hline \multirow{2}{*}{$\begin{array}{l}\text { NR: } \\
\text { SAS: }\end{array}$} & \multicolumn{3}{|c|}{ Not recorded } & \multicolumn{3}{|c|}{ BP: $\quad$ Blood pressure } \\
\hline & \multicolumn{3}{|c|}{ Sedation Agitation Scale } & \multicolumn{3}{|c|}{$r: \quad$ Correlation coefficient } \\
\hline
\end{tabular}

Note. From "Using and understanding sedation scoring systems: A systematic review", by B. De Jonghe, D. Cook, C. Appere-De-Vecchi, G. Guyatt, M. Meade, H. Outin, 2000, Intensive Care Medicine, 26 (3), p.280. Copyright 2000 Springer-Verlag. Adapted with permission.

\section{Reliability of the Sedation-Agitation Scale}

In addition to obtaining data that a sedation-scoring scale is measuring what is intended, it is important to substantiate that the tool is measuring something that can be reproduced. Reproducibility refers to the ability to get the same results by different observers, on different occasions, and/or by similar or parallel tests that 
produce identical or similar results (Streiner \& Norman, 1995). With numerous nurses and doctors assessing sedation and agitation in ICU patients at various times, it is essential that reliability of an instrument be established. Reliability is defined as "the consistency with which a measure discriminates between patients at a single point in time" (De Jonghe et al., 2000, p.276). Like validity there are different forms of reliability. Riker et al. (1999) chose to determine the inter-rater reliability of the SAS. Riker et al. define inter-rater reliability as "the extent to which multiple examinations of the same patient or specimen agree with one another" (p.1326). Only a few sedation-scoring instruments have undergone any testing to determine their inter-rater reliability. Riker et al. reported that the SAS had excellent inter-rater reliability with an inter-rater correlation of $r=0.83$, $p<0.001$ and the weighted $\kappa$ score for inter-rater agreement was $0.92(p<0.001)$. The modified SAS was evaluated with the Harris $(r=0.93, p=<0.001)$ and the Ramsay Scale $(r=0.91, p=<0.001)$, with the authors concluding that the SAS tool was a reliable and valid tool to describe sedation and agitation in ICU patients.

\section{Conclusion}

The SAS has been shown to be a valid and reliable tool for use in the ICU under certain conditions. The validity of the SAS has been determined when evaluated against other sedation-scoring tools. The SAS has been found to be reliable when used by inexperienced and experienced nurses, and in medical, surgical and cardiac ICUs in the USA. It is not clear however, whether the SAS is a reliable or appropriate tool for patients with neurological impairment or whether it is able to assess the responsiveness to changes in sedation over time. Both Riker et al. (1999) and Brandl et al. (2001) did not consider whether the SAS was a reliable tool when used by doctors and nurses. Research to date has not been able to confirm whether nurses and doctors using the SAS in an ICU to evaluate a patient are rating the patient similarly. It is important to know this to ensure these professionals share a common understanding of the terminology as clinical decisions are made based on these observations. This is the focus of this study. 
This literature on the SAS as well as that presented in the earlier chapters sets the scene for the empirical chapters that follow by introducing the various bodies of literature that informed the study design and influenced the formulation of its research question. 


\section{Chapter 5- Quasi-Experimental Methodology and Study Design}

Since Florence Nightingale's time, nurses have expanded their foundation of practice through research that has tested and developed nursing knowledge. The very philosophical notion that nursing is an art and a science keeps nursing at the maximum tension between the sciences and humanism. Thus nurses use and will continue to embrace both quantitative (experimental) and qualitative research designs in order to expand their body of knowledge. Quantitative research usually produces findings that are generalisable to others, whilst qualitative research is specific to a particular person or setting and tends not to be transferable to a wider population (Gillis \& Jackson, 2002). Gillis and Jackson purport that the very core of nursing research is emulated in nurses' every-day practice. Accordingly often nursing research is engaged in the everyday world it has sought to change. For that reason quasi-experimental designs are often a popular choice within nursing research. This research was completed in a natural setting to ensure that the Sedation-Agitation Scale (SAS) was reliable in clinical practice in the Wellington Intensive Care Unit (ICU). The study used a quasiexperimental design to evaluate the reliability of the SAS when used by nurses and doctors.

In order to gain an appreciation of the importance of reliability testing, it is essential to understand the methodological underpinnings of the chosen research design and the foundation of reliability, the true score theory of measurement. This theory will be discussed in the following quasi-experimental methodology section along with the types of measurement error. Errors in measure play a key role in degrading reliability. A discussion of reliability and the types of reliability will also be presented, including the justification for the chosen design. The second part of this chapter presents the actual quasi-experimental study design and method. It is also detailed the aim of the study, the setting, how participants were selected, the data collection tools used, how the data was analysed and ethical considerations of the study. 
Contemporary empirical research such as quasi-experimental designs is increasingly underpinned by post positivism (Clark, 1998). Post-positivism does not discard the truth present in such methodologies that focus on the meanings and experiences of individuals (as in qualitative research). Post-positivism has less ambitious aims than that of positivism, namely of gaining a greater estimation of truth. This is supported by Poole and Jones (1996) who suggest that in research today, there is an acceptance that the determination of absolute truth is an unattainable goal and therefore inferred relationships (causal) are now commonly expressed according to a specified level of probability. Indeed within evidence-based practice, results are always deemed statements of probability.

\section{Quasi-Experimental Research Design}

The word quasi means 'almost' in Latin. The inability to achieve experimental controls in the real world led to the development of a tailored experimental approach; the quasi-experimental designs. Quasi-experimentation are designs that bear some resemblance to true experiments, except that it has not been possible to meet the following criteria; randomisation of subjects to the experimental or control group or an inability to control the timing or nature of the treatment variable (Maas \& Buckwalker, 1989). This is usually because of ethical matters, situational factors in the 'real world' or institutional policies. Quasiexperimental designs are utilised when examining cause and effect associations in natural settings when it is important that particular research is done, despite not having a 'perfect' experimental design.

To some extent quasi-experimental designs have been embraced by nursing. There are times when nurses want the power of an experimental design in making causal inferences, but find it is not possible to conduct an experiment. Nursing does not exist in a sterile laboratory; it is immersed in the real world. Quasi-experimental designs, therefore, often provide the strategies needed to contribute to knowledge of the environment, rather than forgo the systematic study of phenomena for which the use of a 'true' experimental design is not possible (Watts, Jones, Wainwright, \& Williams, 2001). An important feature of 
quasi-experimental designs is they accommodate for the absence of either random assignment of groups or the control group component, by introducing other controls. Rather comparisons are made with non-equivalent groups or with intermittent measurement of the same group; change is then inferred due to treatment effect (Maas \& Buckwalker, 1989). The major challenge for the researcher who uses a quasi-experimental design is in identifying the effects that are the result of the treatment from those owing to uncontrolled extraneous variables. Seminal work by Cook and Campbell (1979) describes in detail various tools and options for controlling extraneous variables that may be a threat to the causal variable of interest. It is beyond the scope of this paper to discuss them in detail, but it is essential that controls are put in place in a quasi-experimental design, to control for extraneous variables otherwise valid conclusions cannot be drawn from the results.

\section{Non-equivalent Group Design}

Quasi-experimental designs split into two general categories. Non-equivalent group designs involve measures of the dependent variable from an experimental and comparison group which are not randomly assigned. The measurement occurs prior to and after the introduction of the independent variable to the experimental group (Cook \& Campbell, 1979). Some designs that fall under this category are arguably not able to permit reasonable causal inferences. These include the one-group post-test only design, the post-test only design with nonequivalent groups and the one-group pretest-post-test design. Cook and Campbell argue that these designs are not adequate to allow robust investigation of causal hypotheses because they fall short of negating other plausible alternative explanations for the results. Then again they suggest any of the following designs do provide interpretable results that rule out most threats to internal validity including the untreated control group design with pretest and post-test, the non-equivalent dependent variables design, the removed-treatment design with pretest and post-test, and cohort designs. Many of these designs can be integrated within a single study thereby significantly enhancing the causal inferences. 


\section{Interrupted Time-Series Design}

Interrupted time-series designs measure results after the introduction of the independent variable. The effects on the dependent variable are measured at multiple time intervals (Maas \& Buckwalker, 1989). The basic time-series design uses a single group of subjects only but some may use different subjects with similar group features. There are a multitude of variations on these designs, including simple interrupted time series, interrupted time series with a nonequivalent no-treatment control group time series and interrupted time series with removed treatment. A time series design may also be integrated with a nonequivalent group design in order to strengthen the interpretability of the study (Cook \& Campbell, 1979).

\section{Advantages and Disadvantages of Quasi-Experimental Designs}

Quasi-experimental designs are effective in investigating hypotheses in natural settings. They also provide a structure for answering questions that may have otherwise been left to skewed analysis, trial and error or conclusions drawn from compromised experiments in which opposing causal hypotheses have not been clearly evaluated (Gillis \& Jackson, 2002). Thus quasi-experimentation provides a comprehensive design, which fuses strong causal inferences, along with being strong on external validity.

Maas and Buckwalker (1989) suggest that quasi-experimental designs can improve generalisability over true experimental designs because as a rule they are less intrusive in natural settings, less artificial and more apt to be representative of the real world of nursing practice. This tends to make them more appealing to nurses active in research, albeit replication of quasiexperimental research is often required to enhance the generalisability and strengthen the reliability of studies through testing and retesting. Quasiexperimental designs aptly use reliability testing within their designs, either by the test-retest method or by correlation computations. Despite the fact these designs may appear to have non-equivalent groups, correlation coefficients have 
indicated reliability in the instruments used in quasi-experimental designs (Poole \& Jones, 1996; Skodol Wilson, 1993).

Riker et al. (1999) used a quasi-experimental design in determining the interrater reliability of the Sedation-Agitation Scale (SAS). Their study was performed in a natural setting in an Intensive Care Unit (ICU) in Maine. A convenience sample of ICU patients was used. Patients were identified as potential participants by the evaluators to ensure one portion of the ICU population was not under represented. If it appeared that one part of the population was not well represented then patients were preferentially recruited from that group in the following week.

Quasi-experimental designs by definition lack the controls fundamental to experimental designs. The main aim of designs examining causal relationships is to provide controls that take full advantage of the effect of the independent variable on the dependent variable, while minimising the effects of extraneous variables and curtailing measurement error (Maas \& Buckwalker, 1989). The key limitation of quasi-experimental design is their lack of control over extraneous variables that threaten the inference that the treatment produced the changes. For example, because of the lack of randomisation, the experimental and comparisons groups may be different in some systematic way (Cook \& Campbell, 1979) which may then be the reason for any measured difference. For that reason it is critical to attempt to rule out other explanations from the findings before surmising that the treatment variable produced the difference (Maas \& Buckwalker). These extraneous variables are known as threats to internal validity or threats to external validity.

\section{True Score Theory and Measurement Error}

Errors in measures play a key role in degrading results from reliability studies, for that reason, to gain an appreciation of the importance of reliability for this study, it is first important to understand the foundation of reliability, the true score theory of measurement. 
True score theory is a theory about measurement. The theory maintains that every measurement is the sum of two components, true ability/score of the respondent on that measure, and random error (Trochim, 2002). When one rates a measure, one observes that score but does not see what is, in effect, on the right hand side of the mathematical equation. This equation is presented below in Figure 1.

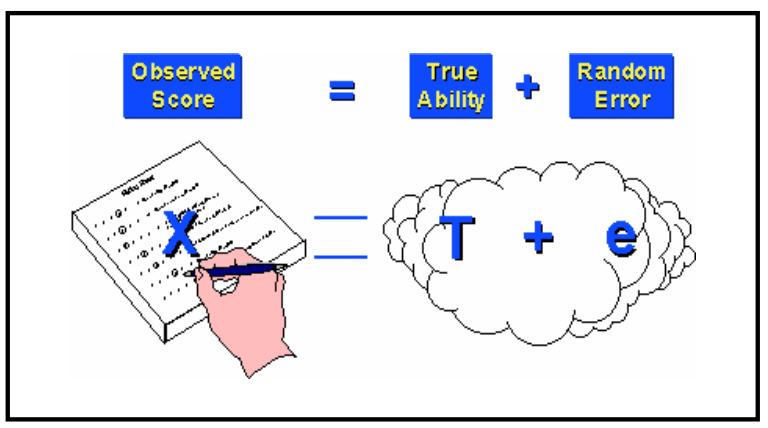

Figure 1. True Score Theory

From "The research methods knowledge base", by W. Trochim, 2002. Copyright 2002 by Cornell University. Reprinted with permission.

This fundamental equation also has a parallel equation at the level of variability of a measure. The equation is $\operatorname{var}(X)=\operatorname{var}(T)+\operatorname{var}\left(e_{X}\right)$. It represents the variability of the measure as the sum of the variability due to true score plus the variability due to random error. True score theory is important to understand as it reminds us that most measurement has an error component. A scale that has no random error (is all true score) is perfectly reliable; a scale that has no true score (all random error) has zero reliability (Streiner \& Norman, 1995). True score theory therefore, provides us with some idea of why measurement models are important.

True score theory is a useful and simple model for measurement, but it does not always provide an accurate reflection of reliability. The theory has an assumption that any observation is made up of the true value plus random error. However, not all error is random (Brink \& Wood, 1989). The error component of the true score theory can actually be subdivided into two groups, random error and systematic error. This is represented in Figure 2. 


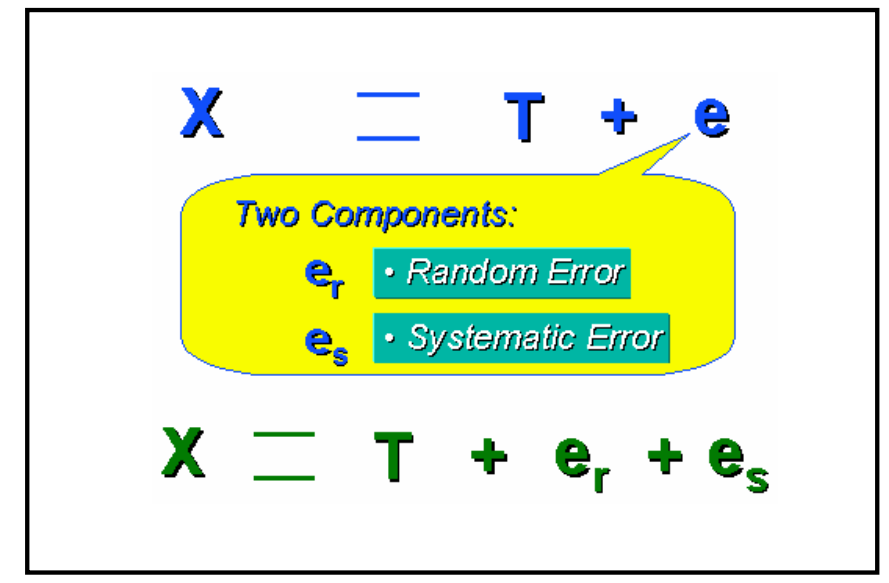

Figure 2. Measurement Error

From "The research methods knowledge base", by W. Trochim, 2002. Copyright 2002 by Cornell University. Reprinted with permission.

Measurement error, whether random or systematic, is problematical because it tends to reduce the power of the relationship between variables, thus weakening the study's findings (Brink \& Wood, 1989). Therefore it is essential that both types of error are controlled to the greatest degree possible.

\section{Random Error}

Random error refers to the random influences that tend to make measurements differ from time to time or they may cause variations in performance from item to item on a single measure (LoBiondo-Wood \& Haber, 1994). Random errors are unsystematic in nature and are the result of a transient state in the subject, in the context of the study or in the administration of the instrument (Brink \& Wood, 1989). For example, a person's mood can fluctuate and affect their performance on any occasion. Thus it may artificially inflate the observed scores for some participants and artificially deflate them for others. Other examples include irritability or tension, or fluctuating environmental conditions such as heat or noise that affect the object measured or the measuring instrument. In considering this research random influences such as these have also been contemplated. Noise is an issue sporadically in the ICU and frame of mind may play a role with the raters, especially if there are patients dying or tragic circumstances occurring in the unit when the ratings are taking place. Random influences are never 
completely eliminated, but efforts can be made to reduce these influences so that the measures are consistent and stable. In this study the researcher was able to monitor the unit environment and know when not to attempt ratings, that is, during extreme circumstances in the unit. This was of paramount importance to maintain the ethical safety of the study.

Random errors add variability to the data by affecting the consistency of the measurements, but it does not affect the average performance of the group (Streiner \& Norman, 1995), as is demonstrated in Figure 3; it is a normal distribution or a bell-shaped curve. Because the reliability of a measure depends on its degree of consistency and repeatability, as the amount of random error in a measure increases, reliability is reduced. Thus as Brink and Wood (1989) suggest, reliability is determined by the degree of random error or true scores in an instrument.

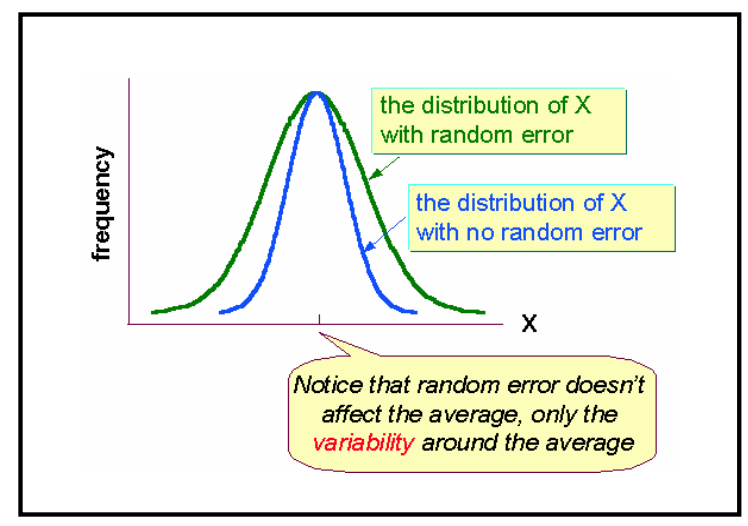

Figure 3. Random Error

From "The research methods knowledge base", by W. Trochim, 2002. Copyright 2002 by Cornell University. Reprinted with permission.

\section{Systematic Error}

Systematic error refers to systematic influences that may distort an instrument's scores. Such error has a systematic bias on measurement events, across the sample, which may influence the validity of the instrument (LoBiondo-Wood \& Haber, 1994). For example, a set of scales that consistently weigh a person $3 \mathrm{~kg}$ less than their actual body weight produces a systematic error. 
Reducing measurement error can be done using complex statistical procedures. More practical ways of reducing measurement error include ensuring any technical equipment used is calibrated as per the manufacturer's specifications. Pilot testing instruments is useful to get feedback from respondents regarding the ease of measure using a tool. This was illustrated in Riker et al. (1999) who revised the SAS after feedback from ICU nurses. Providing adequate training for the observers/data collectors is essential so they do not inadvertently introduce error due to a lack of understanding about the instrument. When Wellington ICU introduced the SAS, it was important that education for the tool was provided for all staff, to ensure they understood the guidelines on how to use the SAS. Measurement error can also be minimised when doing data analysis. All data should be checked for accuracy.

\section{Reliability}

In considering measurement the basis for applying an instrument to a situation is because the measure should provide information about the object of observation (Streiner \& Norman, 1995). For example, the SAS tells us something about the behaviour of a particular patient and their level of sedation. Reliability plays an important role in the selection of instruments for use in a study. It is essential to have tools that are reliable and can provide values with only a small amount of random error. Before evidence can be attained that a scale is measuring what is proposed, it is essential to collate data that indicates the scale is measuring something in a replicable manner. This initial action of gathering evidence on the reproducibility of the instrument is to establish that measurements of individuals on various occasions, or by different observers, or by similar or parallel instruments, produce similar results (Streiner \& Norman).

Reliable instruments enhance the ability of a study to determine causal relationships. Lynn (1989) reveals a number of misuses of the terms reliability and validity that have appeared in nursing journals. She argues there is a much too liberal use of these technical terms with little understanding of their true meaning. Lynn illustrates this by presenting a study which reviewed 191 articles 
published in the journals Nursing Research, Research in Nursing and Health, and Advances in Nursing Science. This review found that reliability estimates for the use of an instrument were only cited in $42 \%$ of non-instrument development studies and in only $60 \%$ of instrument development studies. This suggests that there needs to be a greater awareness by nurses and nurse researchers, of the critical role reliability plays in research. Within nursing research, Lynn (1989) suggests not enough attention has been paid to the importance of reliability and there needs to be more awareness by nurse researchers of the critical role reliability plays in data collection. Hence it is time that further reliability testing of the SAS took place.

Brink and Wood (1989) define reliability as "the degree of consistency and repeatability of the scores on an instrument" (p. 264). It is a rudimentary way of signalling the amount of error, random and systematic, implicit in any measurement. Hence it refers to the extent to which measurement error is curtailed. Reliability is concerned with characteristics such as dependability, consistency, accuracy, comparability and repeatability and these terms are often used interchangeably with reliability (Burns \& Grove, 2001). Reliability cannot be computed because we are unable to calculate the variance of the true scores, but an estimate of reliability can be obtained which is usually expressed in the form of a correlation coefficient. A correlation coefficient of 1.00 indicates perfect reliability and 0.00 indicates no reliability. A coefficient of 0.8 is the lowest value acceptable for psychosocial measurement instruments to be considered reliable (Gillis \& Jackson, 2002). For example, if a reliability coefficient is reported as 0.83 , then $83 \%$ of variance in the scores is due to variation in true differences between the participants on the concept under measurement. The remaining $17 \%$ of the variance may be due to other variance in the measurement such as chance.

One other important consideration of reliability is that estimates of reliability are specific to the sample tested. High reliability values reported on an established 
instrument do not guarantee that it will be reliable in another sample or with a different population (Burns \& Grove, 2001). Therefore, it is important that the reliability of the SAS is determined for use in the Wellington ICU patient population. Whilst the tool was tested in the United States of America (USA) for reliability by Riker and colleagues (1999) their sample population and the demographics of their raters is different from that of the Wellington ICU. Riker et al. used eight experienced ICU nurses. This study uses nurses and doctors working in Wellington ICU irrespective of their level of experience. Riker and colleagues' sample population was from a general medical and surgical ICU whilst in Wellington ICU the general population includes cardiothoracic surgery and neurosurgery. For these reasons it could not be assumed that the Riker et al. study was generalisable to the New Zealand ICU context, consequently the SAS tool was retested for reliability on a different population.

Mowinski Jennings and Rogers (1989) in their discussion also highlight a misconception about reliability that is sometimes seen in research. Specifically that the use of a large sample size is a 'full proof' way of guaranteeing that empirical evidence is credible. Large sample sizes are often required to meet primary mathematical assumptions inherent in statistical analysis; to have sufficient power to detect causal relationships among variables. Nonetheless, equally important and completely separate, is the issue of reliability. Increasing a sample size will not correct for error; reliability, not sample size is the key indicator of error. Only through testing that an instrument is as consistent as possible can the effects of error be modified (Mowinski Jennings \& Rogers).

\section{Types of Reliability}

There is no single measure of reliability that is always preferable. The choice is reliant upon the intended function of the instrument (Karmel \& Karmel, 1978). Reliability has four main attributes; stability, equivalence, internal consistency and parallel form. 
Stability is concerned with the consistency of recurrent measures of the same instrument. Stability of an instrument is of interest to researchers, when they want the instrument to measure a concept time after time (LoBiondo-Wood \& Haber, 1994). This is also known as test-retest reliability (Figure 4).

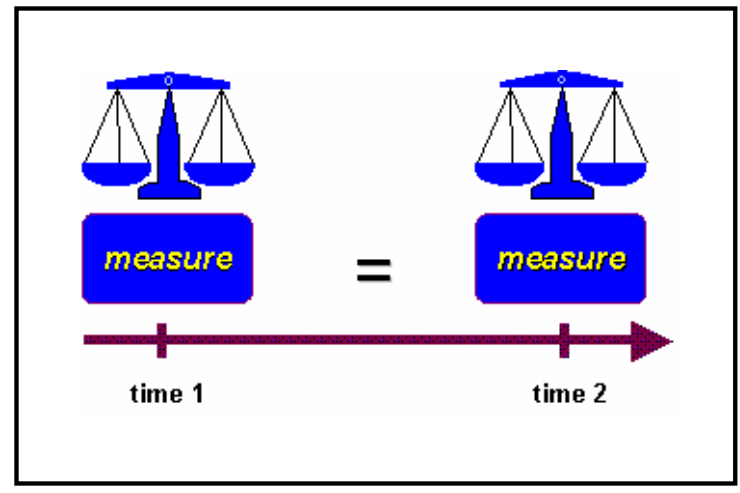

Figure 4. Test-Retest Reliability

From "The research methods knowledge base", by W. Trochim, 2002.

Copyright 2002 by Cornell University. Reprinted with permission.

There is an assumption that the feature being measured does not change over time (between the measurement points). As a result the concept must be relatively stable over time. With the test-retest procedure, the researcher is concerned with the consistency of the measure in the same group from time-one to time-two. For this reason, cognitive measures, such as hope, anxiety and coping, that may change rapidly, are not useful for such an instrument (Burns \& Grove, 2001). The amount of time allowed between measures is critical. If the notion being measured does change, the test is not a measure of reliability. In fact if the measures stay the same even though the concept being measured has actually changed, then the instrument may lack reliability.

The test-retest reliability scores are compared and are usually expressed by a Pearson $r$ correlation coefficient. The higher the Pearson coefficient the more stable the instrument is assumed to be. There are however, problems associated with testing in this way as Brink and Wood (1989) illustrate. Researchers are often able to attain a measure of the idea at a set point in time, but it can be very expensive and impractical to obtain measures at various points in time. As discussed previously, a lower correlation may indicate that the concept has 
changed rather than show a low reliability. The longer the time interval between tests, the more likely it is that the concept has changed. Brink and Wood also suggest that test-retest can lead to depressed reliability due to reactivity. If an individual's belief concerning a concept is measured at time-one, they may then become sensitised and may show a change at time-two. Trochim (2002) asserts that test-retest is useful in experimental and quasi-experimental designs that use a no-treatment control group. The problem with this, however, is that you don't get any information about reliability until after the data is collected from the posttest. If the reliability is then low, the findings are then affected considerably.

Equivalence refers to the amount of agreement or consistency among two or more different observers using the same measurement tool (Gillis \& Jackson, 2002) (Figure 5). Riker et al. (1999) used inter-rater reliability testing in their evaluation of the SAS.

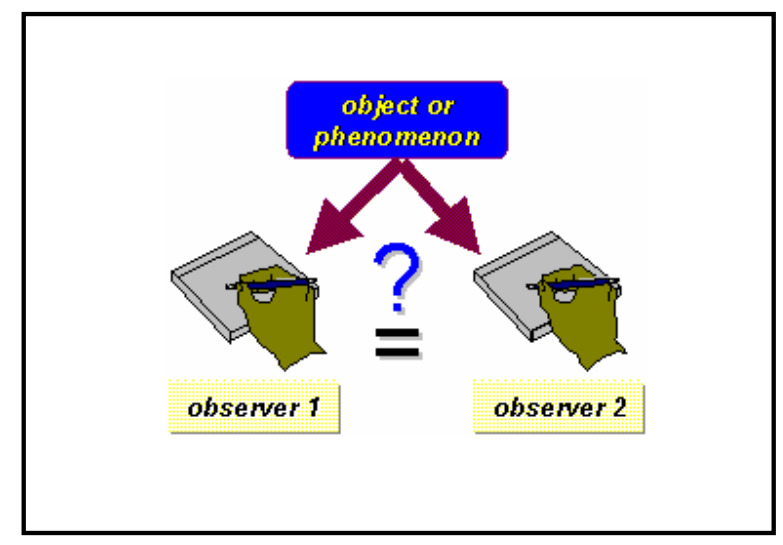

Figure 5. Inter-rater Reliability Test

From "The research methods knowledge base", by W. Trochim, 2002.

Copyright 2002 by Cornell University. Reprinted with permission.

Inter-rater reliability values must be accounted for in any study where observational data is collected or judgments are made by two or more data gatherers. There are two ways of determining inter-rater reliability. Firstly, two or more observers independently observe and log the same event using an instrument developed for the study. This was the design of the Riker et al. study. The observers were not able to communicate with each other. An alternative is 
the same rater observes and records an event on two separate occasions (Brink \& Wood, 1989).

Inter-rater reliability is one of the best ways to estimate reliability when the measure is an observation, but it requires multiple raters or observers. It is useful with a team of raters, in which you wish to establish that they yield consistent results. For this very reason this study was a reliability study. It is not however titled an inter-rater reliability study because the study distinguishes between the raters by identifying them as nurses or doctors. Inter-rater studies consider how similar people are rating without taking into account the category of the rater. Inter-rater testing assumes that on average the raters are the same, there is no concept of there being different average values for the raters (personal communication, Gordon Purdie, [Biostatician] $3^{\text {rd }}$ April 2003). Another aspect of this, however, is whether two different professional groups rate similarly. In this study I am differentiating by separating the doctors and nurses to determine whether they rate patients' similarly using the SAS.

It is necessary that raters are appropriately trained to the definitions of the behaviour to be observed, otherwise random error is amplified with confusion and misunderstanding in the interpretation of the instrument. Reliability between raters can be improved, or random error reduced by discussions about disagreements in ratings and deciding on rules for when to use a ' 3 ' or a ' 4 ' for grading a specific item. This is an important part of the education that needs to take place. Riker et al. (1999) also used this in their reliability study when training the raters on the use of the SAS. In this study all the staff working in the Wellington ICU have been trained previously in the SAS and use it daily in their practice.

Inter-rater reliability studies assess the reliability or consistency of the observer rather than the reliability of the instrument (LoBiondo-Wood \& Haber, 1994). Burns and Grove (2001) argue that when raters know they are being watched, 
their accuracy and consistency are greatly improved, from when they believe they are not being watched. As a consequence inter-rater reliability declines when raters are assessed covertly. Gillis and Jackson (2002) also suggest that inter-rater reliability testing needs to be determined several times in a study as factors such as fatigue, hunger, boredom or familiarity may have an effect on the rater's ability to accurately rate observations. Future studies of the SAS may wish to investigate this. I do not believe that these were significant influences in this study as the staff in Wellington ICU had been working with the tool for only a few months.

The commonly used statistic for describing inter-rater reliability is kappa. Kappa is an estimate of the degree of agreement between two administrations of an instrument after taking into account the amount of agreement that may have occurred by chance (Peat, 2002). As for other correlation coefficients, a kappa value of zero represents only chance agreement and a value of one represents perfect agreement. Peat also recommends that if there are three or more possible reply categories in an instrument, as there are in the SAS with seven, then a weighted kappa must be calculated. Kappa takes into account only total agreement and does not provide part recognition for responses which may differ by only one or two categories. Weighted Kappa is an extension that does consider partial agreement (Streiner \& Norman, 1995).

Parallel forms of reliability can only be tested when there are two equivalent forms of the same instrument in existence (LoBiondo-Wood \& Haber, 1994) (Figure 6). It is similar to test-retest reliability in that the same sample group are tested within a particular time frame, but it differs because a different form of the instrument is given to the sample also. In this way each instrument whilst measuring the same thing is constructed so they can be used independently of each other and are considered parallel measures. Parallel forms tend to also provide a measure of stability and equivalence. The correlation between the two parallel forms is the estimate of reliability. 


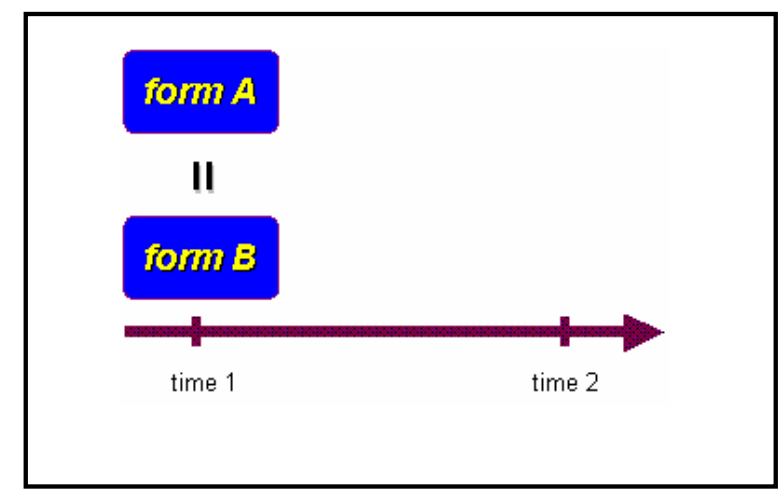

Figure 6. Parallel Reliability Test

From "The research methods knowledge base", by W. Trochim, 2002. Copyright 2002 by Cornell University. Reprinted with permission.

An example of this is in the Riker et al. (1999) reliability study. Three instruments that all measure sedation and agitation in ICU patients, were tested on the same patients by the experienced ICU nurses. The scores were then compared. All three tools indicated high correlation coefficients with each other. The difficulty with parallel forms is that you need lots of items that reflect the same construct. It can be very challenging to find several instruments that measure the same thing.

Internal consistency is also known as homogeneity. Internal consistency uses a single measurement instrument administered to a sample on one occasion to estimate reliability (Trochim, 2002). The reliability is determined by estimating how well the objects in the instrument measure the same variable. A homogenous instrument contains items that are strongly correlated to each other (Burns \& Grove, 2001). Taken together, all the items in the instrument reflect the same underlying concept; the research can sum the individual scores to get a total score that reflects the construct (Gillis \& Jackson, 2002). Regularly nursing research deals with phenomena that are multi-dimensional, for example; job satisfaction, burnout, health status and social supports. In these, researchers must establish separate internal consistency estimates for each dimension of the concept. Internal consistency can be established by several different methods. The primary approach is the split-half reliability. This involves obtaining a testretest reliability without dispensing the test twice. It entails splitting the scale in half and making a comparison. The practice uses a correlation (for example, Cronbachs alpha) that is based on the consistency of responses to all the items 
of the instrument that is administered at one time. This is applicable to scales, for example the Likert scale, with multiple responses, used in particular in measuring psychosocial variables (LoBiondo-Wood \& Haber, 1994).

\section{The Relationship between Reliability and Validity Testing}

Reliability and validity are inextricably bound, although they are often thought of as separate ideas. Poor reliability will compromise the validity of an instrument as it limits accuracy and the conclusions that can be drawn from the results. In contrast, a valid instrument may also have a degree of measurement error (Peat, 2002). Brink and Wood (1989) argue that consistency among the items of an instrument does not necessarily mean that the tool is measuring the intended concept, only that whatever it does measure, it measures precisely. Consequently the tool is reliable but not valid. An instrument that has low reliability does not represent anything systematically and therefore cannot represent the concept as intended. Therefore for a measure to be valid, it must also be reliable.

Reliability is a necessary but not sufficient condition for validity. The validity of an instrument is limited by its reliability. For that reason less confidence can be placed in scores from tests with low reliability coefficients (LoBiondo-Wood \& Haber, 1994). In any research study, it is important to incorporate steps to improve reliability (by reducing measurement error) and validity. These and other

practices tend to minimise both bias and measurement error thus lead to the reporting of reliable results.

Researchers in the health sciences commonly face a need to measure something which has not been attempted before. For example, pain in children, return to function post-myocardial Infarction, speech difficulties of stroke patients, or clinical competence of medical staff versus nursing staff. The complexities and questions raised in developing such tools span from the straight-forward, 'How many boxes do I put in the tool?' to the complicated, 'How do I ascertain whether the tool is measuring what I wish it is?' (Streiner \& Norman, 1995). The second 
section of this chapter presents how the methodology was applied in this research design.

\section{Study Design and Method}

\section{Research Question}

A quasi-experimental design was used in a reliability study of the SedationAgitation Scale (SAS) in Wellington ICU. The research sought to answer the question, in the real world, with minimum exclusion criteria and a randomly selected ICU population, do nurses and doctors rate the SAS similarly?

\section{Null Hypothesis}

The null hypothesis was; there is no difference in the ratings by nurses and doctors using the SAS.

\section{Aim of the Study}

The aim of the study was to determine the reliability of the SAS when used by staff in a tertiary level general ICU. It was important to determine the reliability of the SAS in a general ICU population and to determine whether nurses and doctors rate patients' level of sedation similarly. If nurses and doctors are not able to have a mutual understanding of the terms used within the SAS to describe sedation, a patient's behaviour or a means of determining a patient's level of sedation then clinical decisions and sedation needs of individual patients are not able to be based upon the ratings using the SAS.

\section{Ethical Approval}

As part of applying for approval from the Wellington Ethics Committee (see Appendix $B$ for confirmation of ethics approval), discussions were held with the Chairman over whether individual consent from patients or their representatives would be needed. He indicated that this would not be necessary as the focus of the study was with the staff in the ICU. This study was a reliability study, which was auditing a tool already in use in the ICU, to determine whether it was a reliable tool for ascertaining the level of sedation for an individual patient at a 
given point in time. The observations made were already routine, accepted practice observations and there was no intention to alter treatment with this study. The reliability or consistency of the staff was being tested, not the patient.

Notices were placed in the ICU and the family waiting area to inform families that the study was taking place and information pamphlets were made available to families to provide more information, including my contact details should they wish to discuss the study further (Appendix $C$ ). This was done so families would not be distressed if three staff appeared at their loved-one's bed space, without warning, which could potentially be distressing to them. It was essential that the lead investigator along with the primary nurse kept the families informed of what was about to occur, if they were present.

Prior to the commencement of the study all staff willing to participate were required to sign a consent form and provide demographic and professional data about themselves (Appendix D). Details included in the consent form were; the purpose of the study, participants' right to decline to participate without prejudice, guidelines for the storage of the data collected and demographic data about the individuals. After consultation with the Maori Health Unit at Capital and Coast District Health Board (C\&CDHB) ethnicity data was added to the consent form.

As the researcher, staff have a professional relationship with me in my capacity as Clinical Nurse Specialist (CNS) of the ICU. I acknowledge that some staff may have found it difficult to express their wish not to participate. To avoid staff feeling coerced there was a three week period during which staff could consider whether to consent to participate, prior to data collection commencing. Staff who did not wish to participate would not return a consent form. Any communication with staff regarding the consent forms during this time was of a general nature and no individuals were approached. No identifiable names or dates were collected on the data sheets, so staff or patients were not able to be identified individually in 
the analysis. All data remained in a locked filing cabinet, in a locked office, that only the researcher had access to. Any computer work was password protected.

\section{Safety}

In using the SAS in this study, there was no anticipated impact on patient safety. The SAS was not intended to be used in the ICU as a basis for clinical decisionmaking, until such time as this study determined its reliability.

The Wellington Ethics Committee raised concerns with regard to the possibility of psychological risks to staff (Appendix E). I had not considered there might be a risk to the staff. Nonetheless an alteration was made to the staff consent form and access to named senior nursing and medical personnel was arranged so they could be available for participants if they did not feel comfortable approaching myself with any concerns. Wellington Hospital also has an Employee Assistance Program (EAP) which offers counselling and support to staff with any issue. All staff have free access to this program and all staff within ICU have information on how to access this program.

Part of my role as the CNS and researcher in the ICU is to ensure that all patients were continuously monitored. As an experienced ICU practitioner, I was also able to recognise when it was not an appropriate time to be doing ratings. If emergency situations were occurring in the ICU, or staff were pressured or extremely stressed, the researcher had the experience to make a judgment that these were not appropriate times to be doing data collection. In clinical practice emergencies in the ICU sometimes mean that routine observations and assessments may be delayed.

\section{Setting of the Study}

The study took place from August 2003 to the end of September 2003 in a tertiary general ICU that covers surgical, medical, paediatric, trauma, cardiothoracic, neurosurgical and renal intensive care. The ICU has 14 beds, with only 12 operational at any one time and an annual average occupancy of 11 . 
Data collected in the Wellington ICU for the 2002-2003 financial year demonstrated 1307 admissions, 653 of which were elective surgical admissions and 654 were acute admissions. The average length of stay (inclusive of cardiothoracic surgical patients) was 24 hours and the maximum length of stay was 201 days. The unit is an open planned unit of 10 beds plus two single rooms and a two bed isolation unit.

The staff consist of 59.5 full time equivalent staff nurses ( 80 staff nurses), six Clinical Nurse Co-ordinators (CNC), one Clinical Nurse Preceptor (CNP), one Nurse Educator, one Clinical Nurse Specialist, seven registrars and five ICU consultants. There was no master database or information available on the staff characteristics such as gender, ethnicity or qualifications. The consultants rotate through being 'on call' and they oversee the registrars, as well as assuming the overall medical responsibilities for patient care. While the consultants were permanent employees of the ICU, the registrars worked on a 6-month rotation; coming from the medical, surgical, anaesthesiology and emergency specialties. They would be responsible for the care of the patients in the absence of the consultant. There were usually two registrars on each shift during the day and one in the unit over the night. The registrars were each responsible for all the patients in the ICU, they would share the workload and did not split the patient load between them. The ICU also has a 1-year registrar training position in intensive care.

The registrars in Wellington ICU are not permanent staff members of the unit as they rotate through various specialty areas of practice, however for the purpose of this study they are treated as such. The ICU consultants and nurses are permanent staff of the ICU, with specific knowledge and skills in intensive care. The CNCs are responsible for the efficient running of the unit on a day-to-day basis; they do not take a patient load. The Nurse Educator is responsible for running the advanced practice program in intensive care that is offered nationally to ICU nurses. The CNP is responsible for the education of the nurses; both formal educational opportunities and support for staff on a daily basis in the unit. 
The CNS (the researcher) has specialised skills in critical care, can take a patient load, co-ordinate quality assurance, research and educational activities and has the overall responsibility for maintaining standards of care.

The nursing positions are filled by full-time and part-time staff. Most nursing staff work a 12-hour shift pattern rotating between day and night duty. There are also a small number of staff working an 8-hour shift pattern. Throughout the course of a shift, a nurse is responsible for the care of one patient. The intensive care unit operates on a 1:1 ratio of nurses to ventilated critical care patients, however there are times when a nurse may care for two unventilated patients depending on the demands of their care and the severity of their illness. When critical care nurses from other units arrive in the ICU they are placed on level $2 \mathrm{a}$ of the Clinical Career Pathway (CCP) until their level of competency and experience is determined. After a period of approximately six months these staff may then apply to move up the CCP.

C\&CDHB has four levels to the CCP (RN1-RN4), with specialist positions surrounding and supporting these levels. The practice requirements for each level and for the process of progression are clearly defined. Each level of the CCP has specific competencies, criteria and attributes that have to be met before applying for the next level (Capital Coast Health, 1998). At one end of the continuum is the RN1, defined as a novice who is usually a new graduate staff nurse. At the other end is the RN4 which is described as a nurse with expert skills and knowledge. The Wellington ICU does not take RN1 nurses and tends to employ staff that have a minimum of two years post-registration acute experience. Accordingly nurses employed in the unit start on level $2 a$ of the CCP and progress from there.

\section{Staff Eligibility}

All permanent members of staff working in the ICU had the opportunity to be participants in the study, including registered nurses, ICU registrars and consultants. Agency and casual resource staff were not eligible as they are not 
usually familiar with the SAS tool. New staff that were within their 6-week orientation phase at the time of the study were excluded because during this phase they are learning to use the SAS. Once their orientation was complete they were welcome to participate. The registrars commenced work in ICU three months prior to the commencement of data collection. Their orientation also included training in using the SAS. This was the same training that nursing staff had received four months earlier.

There was an assumption within this study that the primary nurse caring for the patient would have consented to participate in the study. Should this not be the case, another nurse would take their place in the assessment so that a rating of the randomly selected patient could proceed. I believe this approach was fair as the staff in the ICU had indicated to me that they were keen and interested in the study. Nurses routinely step in for each other in the clinical practice setting, covering meal breaks, rest room breaks or when the nurse needs to talk to a family privately, and team work is encouraged within the unit so this should not affect the reliability of the results. The medical staff on any shift do not allocate specific patients to specific doctors, for that reason they must be equally familiar with all patients. Whilst they have a 'distant relationship' with the patient at the bedside, they are clinically responsible for all patients in the unit.

\section{Patient Eligibility}

All patients receiving sedation, over 16 years of age, no matter their length of stay or diagnosis in the ICU were potentially eligible for the study. A decision was then made to assess their eligibility for the study based on the following criteria. Paediatric patients were excluded, as the adult SAS tool is not appropriate for the developmental age of a child and has not been tested in children. Any patient who had already had a rating assessment was excluded from further ratings to minimise confounders. Patients who were deaf or could not speak English were also excluded as communication is a key component to the SAS. Patients who had procedures performed on them (such as turns, airway suctioning, or line insertion) or had received a bolus neuromuscular blockade in the 10 minutes 
prior to a rating were also excluded because the neuromuscular blockade may still be in the patients' system or the patient may just be settling after stressful procedures had been performed. However these patients could be reconsidered for the study at a later time.

\section{Data Collection Tools}

The consent form (Appendix D) which staff participants completed asked for specific demographic details about the individuals. These are presented in Table 5. The details were collated on a database that only the researcher had access to.

\section{Table 5. Staff Participant Particulars}

Staff ethnicity
C\&CDHB CCP level
ICU specialty course
No. years worked in any ICU
No. years worked in Wellington ICU
Previous experience with any sedation-scoring tools
Profession identification

The data collection tool was pre-tested in the ICU to check that it could be completed efficiently and would provide the information required (Appendix F). The data collection tool collected patient details and staff assessment data as summarised in Table 6.

Table 6. Data Collected on the Data Collection Tool

\begin{tabular}{|l|l|}
\hline Patient age & Intubated or extubated \\
Patient gender & APACHE II or Tu score \\
Patient ethnicity & Dose \& mode of sedative delivery \\
Patient length of stay & Staff unique identifier No. \\
Patient diagnosis & Patient SAS scores \\
\hline
\end{tabular}


The APACHE II score and codes (Appendix G) is an ICU prognostic scoring system used in Wellington and internationally, to determine potential outcomes of patients, based on physiological indicators on admission to the ICU. Data collected includes temperature, blood pressure, respiratory rate, renal function, haemoglobin level, arterial blood gas, white blood cell count, Glasgow coma scale and chronic health history. This system provides a benchmark for ICUs of similar size and population when considering outcomes and contemporary clinical management of their patients. APACHE scoring has been shown to have value in predicting mortality, infection and multiorgan failure (Knaus et al., 1991). APACHE II scores are often reported in international critical care studies to help represent the population of the ICU and for comparison with previous research. The maximum possible APACHE II score is 71 . A score of 25 and above is associated with significant mortality (Willatts, 1990). APACHE II scores are not collected on patients that are in the unit less than 24 hours as these patients are either too critically ill and do not survive or are not as sick as anticipated and recover quickly to be transferred to a ward or discharged home. For example, people who have recreational drug overdoses or those requiring post-operative monitoring.

The Tu score is the cardiothoracic equivalent of the APACHE II score and is also a risk prediction score. It is a six variable risk index (age, sex, left ventricular function, type of surgery, urgency of surgery, and repeat operation) to predict inhospital mortality, ICU length of stay and overall post-operative stay in days after cardiac surgery (Tu, Jaglal, \& Naylor, 1995). Collecting this data allows for comparisons with cardiothoracic populations in ICUs internationally. Wellington $I C U$ is a unique unit in that its population consists of two very different groups of patients, both general ICU and cardiothoracic. Like the APACHE II score the Tu score does not predict the quality of outcome following surgery. The Tu score like other predictive scores has also been identified by Weightman, Gibbs, Sheminant, Thackray and Newman (1997) as having insufficient specificity and sensitivity to identify specific individuals who will encounter an adverse outcome. 


\section{Method of Data Collection}

Each staff participant was allocated a unique identifier number. The researcher is the only person who knows the identity of the participants and their demographic details. If staff provided details of part years of work experience, then less than half of a year was rounded down to the whole number below and if it was greater than or equal to a half-year it was rounded up to the next full year. For example 3.3 years was rounded down to 3 years and 4.5 years was rounded up to 5 years.

Small modifications were made prior to the commencement of data collection. It was determined that only sedatives given in ICU were recorded and information about sedatives given in an operating theatre were excluded. It was also decided to collect information on the exact dose and concentration of the sedative administered. The data collected was of importance for describing who the participants were and for any further exploration of current practice at a later date. The researcher completed the data collection tool to minimise error and time for each rating, the doctor and nurse involved in the rating then handed their independent scores to the researcher and watched (to confirm accuracy) as they were individually transposed onto the data collection sheet.

It was necessary to collect this data to allow a general description of the ICU patient population in which the study took place. It also allowed for comparison with Riker et al. (1999) study. The APACHE II scores, diagnosis codes and Tu scores were automatically calculated by the ICU's admission computer database. If a patient was rated before an APACHE II score or Tu score was calculated, which could happen if the rating was done on the day of admission, this data was accessed retrospectively from the database.

The ratings occurred during work time for the staff and due to the numbers of staff and patients, it was anticipated that each nursing staff member may only do 1-2 ratings during the course of the study. It was likely that medical staff would do more ratings, however as there were only 12 doctors in total compared with a 
potential pool of 80 nurses and not all would be available at the time of ratings due to shift patterns. This difference in expected numbers of ratings was not seen as problematic because the study was being performed in a natural setting and the composition of the ICU is such that this will always be the case in clinical practice.

\section{Process of the Ratings}

The patients were randomly selected for the study. Each day the patients' identification stickers were placed in unmarked envelopes of similar size. One envelope was randomly chosen, by the ICU ward clerk. Notes were reviewed and patients who met the exclusion criteria were put aside and a new envelope chosen. The ratings took place at times set at the discretion of the researcher, to ensure the potential pool of raters could not anticipate when these would occur.

The random selection of patients was built on the assumption that most staff had consented to the process prior to the commencement of data collection and therefore the primary nurse would be one of the raters. The staff raters were the nurse and doctor caring for an individual patient. Hence a convenience sample of raters was used. The process of randomly selecting patients could mean some staff that had consented to participate in this study may not actually complete a set of ratings for the purpose of this study. Their participation was dependent on the patients selected. The researcher was not one of the raters therefore ensuring I did not introduce bias to the data collection phase.

Once a patient was selected, the primary nurse and doctor on duty were located and identified and a check was made to see that they had consented to participate. Once this was confirmed they were approached to complete ratings. The nurse-doctor ratings were carried out simultaneously and were not allowed to communicate with each other during the ratings process. The staff raters observed the patient for 30-60 seconds, taking note of spontaneous activity and tolerance of mechanical ventilation (if present). This was then followed by the procedure outlined in Table 7. 
Table 7. Process for Sedation-Agitation Scale Assessment

1. The primary nurse called the patient's name for up to 3 times (progressively louder) to assess their wakefulness. If there was no awakening to verbal stimulation,

2. Then the primary nurse gently shook the patient, by the shoulders for up to 3 seconds, to stimulate them (except in patients with suspected or known spinal injuries). If there was no response,

3. The patient's trapezius muscle was squeezed bilaterally for up to 3 seconds.

4. If the patient awoke to any of these, the primary nurse would ask the patient to squeeze their hand (repeated 3 times) to assess their response.

This simple procedure is routine in assessing the neurological state of patients in Wellington ICU, therefore the ratings were done where possible at the same time that a set of neurological observations were expected, preventing doubling up of assessments. Before leaving the patient, the raters independently wrote their SAS score on a piece of paper then watched as their score was transposed by the researcher onto the data collection sheet. It is acknowledged that it could take some time to get the raters together in order to do a rating, however, once that was achieved the whole patient assessment took less than one minute. Each nurse or doctor was not aware what their colleague's rating was. It became apparent soon after commencing the study that the SAS integration into practice was emerging which the researcher had no control over. It therefore made the reliability testing of the SAS essential and timely.

\section{Data Analysis}

The SAS is ordinal in nature; it has seven defined levels and was treated as continuous data for analysis purposes. Data analysis was made under the guidance of a Biostatistician from Victoria University of Wellington, Health Services Research Centre. Guidance was given on the issue of number of 
ratings, appropriate statistical analysis, and the appropriateness of the research design to the research question. Provisional analysis took place after two weeks of data collection to ensure that the data captured was appropriate. Early estimates of sample size suggested 120 patients would need to be evaluated. On review of the first 50 patients there was such homogeneity of the data that Biostatician advice was that a further two weeks of patient data is all that would be required. Descriptive statistics were used to present demographic data on the raters and to evaluate representation of patients. Patient diagnostic groups were evaluated using frequencies and proportions. Measures of central tendency and dispersion (mean, median and sd) were used on length of stay, age, years of staff experience, the APACHE II and Tu scores.

A factor analysis using Kappa was used to estimate the proportion of staff agreement between two administrations of the tool. Kappa takes into consideration the amount of agreement that has occurred by chance and estimates the proportion of variance that can be considered as between-rater variance (Peat, 2002). Chance agreement corresponds to a Kappa score of zero while absolute agreement is represented by a value of one. It is not possible to get perfect agreement in a natural research setting therefore this study assumed an acceptable level of statistical significant agreement was equal to 0.95. Peat (2002) recommends a weighted Kappa statistic ( $\mathrm{K}$ ) for analysis of an inter-rater reliability study when there are three or more possible reply categories such as when using the SAS tool. In this statistical analysis two or more ratings away from the first response contribute considerably compared with those that are one away from the initial rating. Riker et al. (1999) in their study used the weighted $\mathrm{K}$ as it corrected for chance agreement and incorporated the magnitude of disagreement when comparing the assessments of the raters. De Jonghe et al. (2000) also used weighted $\mathrm{k}$ for their analysis of inter-rater reliability, in their systematic review. Different authors have different interpretations as to what is a good level of agreement (Altman, 1991; Streiner \& Norman, 1995). Altman provides one interpretation of kappa (Figure 7). 
- Poor agreement $=<0.20$

- $\quad$ Fair agreement $=0.20-0.39$

- Moderate agreement $=0.40-0.59$

- Good agreement $=0.60-0.79$

- Very good agreement $=0.80-1.00$

Figure 7. Interpretation of Kappa

Data analysis took place using version 10 of the Statistical Package for the Social Sciences (SPSS) and $\mathrm{k}$ was calculated using software Analyse-it $\circledast$ for Microsoft Excel version 1.69 (2003). Where necessary some data was regrouped for analysis. In doing this, consideration was given to maintaining the integrity of the data and its definitions. For example some ethnicity data was regrouped from 16 categories to four, New Zealand/Pakeha, Maori, Pacific Island and 'Other'. Some regrouping also occurred in presenting the years of experience of the staff participants, to less than or equal to three years, to allow for comparison with Riker et al. (1999). Further analysis and tests for statistical significance of patient variables required 2-tailed t-tests for parametric data and Chi-square for nonparametric data. This analysis could only be completed on variables with cell sizes greater than five therefore not all variables could be evaluated. Statistical significance for this study was defined as $p=\leq 0.05$. Tables and figures are used to illustrate complex data in a meaningful manner.

\section{Rigour of the Research}

This research used a similar design to Riker et al. (1999) thus the researcher was aware of some limitations and able to accommodate these. Procedural matters to do with the research were able to be refined. All staff were trained in the use of the SAS and it is now integrated into the orientation program of new nursing and medical staff in the ICU. This would help to reduce measurement error and improve the validity of the study and reliability of the SAS. 
The SAS is a clinical tool that will be used to guide practice in the Wellington ICU. Staff did not know when they were going to be asked to perform a set of ratings for this study therefore they could not pre-prepare. I do not believe the integrity of the staff was an issue, during the research, in 'guessing' the ratings to please the researcher. There was no reason for the staff to do that when the tool was already in use. The tool comes with a set procedure for evaluating patients which had been developed by Riker et al. Thus all staff participating in the study were clear in how the ratings would take place. The procedure was no different to the assessment that is used for a Glasgow Coma Scale (GCS) evaluation in a critically ill patient.

Finally entry and exclusion criteria for both patient and staff participants were unambiguous and were based on the previous experiences of Riker et al. and Brandl and colleagues (2001). Patients were randomly selected to the study to ensure there was a heterogenous range of patients that reflected the real world of Wellington ICU.

\section{Conclusion}

This reliability study is particularly interested in the concepts of internal consistency and equivalence as it is essential that the different observers (nurse and doctors) using the SAS tool will yield the same results and that these results are consistently obtained on repeated use of the tool in the ICU. It is essential to have accurate data which is the result of precise measurement tools, representative samples and a tightly controlled study design to ensure rigour. It is also important that on embarking on a quantitative study that the research question fits the design. Expert advice is fundamental to this process. Selecting a quantitative design requires the use of statistical analysis programmes so consequently a novice researcher requires constant supervision and support from professionals. This may be an unanticipated and extra resource that is necessary. The time frame for completing this thesis would have been unachievable without this expertise to guide the researcher. 
This quasi-experimental study aimed to determine whether doctors and nurses using the SAS rated patients similarly in the natural setting of an ICU. This would be determined with a reliability study in the Wellington ICU. The study was a similar design to previous studies completed using the SAS and followed the same ratings procedure. Data were to be analysed in a similar manner using weighted Kappa. However further analysis of variables would also be undertaken using chi-square and t-tests. The following chapters examine the findings of the reliability study, and provide an interpretation of the results and their significance to clinical practice and the growing knowledge about the reliability of the SAS. 


\section{Chapter 6- The Findings}

This chapter presents the findings of the research. This study was performed in a general Intensive Care Unit (ICU) at Wellington Hospital using a quasiexperimental design. The aim of the study was to determine the reliability of the Sedation-Agitation Scale (SAS) when used by doctors and nurses in this unit. This chapter describes the sample population in the study and provides results of the data analysis. It presents the demographic profile of staff in the study including ethnicity, years of experience and level of nursing education. Gender, ethnicity, length of stay and age is also presented for the patients. Acute Physiology and Chronic Health Evaluation (APACHE) II and Tu scores and codes are presented for the ICU patients along with details of the type and mode of sedation used. Importantly it reports findings of $K=0.82$, indicating very good reliability.

Forty eight nurses and seven doctors agreed to participate in the study. Of those 48 nurses, six nursing staff consent forms were offered once data collection had commenced. Once data collection commenced the awareness of the study increased in the unit and further staff approached me with completed consent forms. These individuals' patients had not been randomly selected prior to them handing in consent forms. I did not consider this an issue for the study as they were few in number and I had expected that some staff would overlook consenting by the date requested on the consent form. No reminders were provided to ensure staff freely consented. Staff who had not consented to the study did not participate in any ratings. No new members of staff consented following completion of their orientation as data collection had concluded by this time.

Seventy patients were randomly selected for this study and once they had been rated were not eligible for further ratings. The number of ratings completed each day varied depending on the availability of new (not previously randomly selected) patients and the state of the ICU. If the ICU was busy, ratings did not 
occur. The number of paired ratings ranged from 0-4 patients each day over an eight week time span. The time of day that ratings were completed ranged from 0600 to 2030 hours; no ratings were done in the middle of the night.

\section{The Staff Sample in the Intensive Care Unit}

Of the 92 eligible staff, 55 (60\%) consented to participate in this study; $48(60 \%)$ nurses and seven (58\%) medical staff. All seven doctors took part in ratings, however only 35 of the 48 nurses ( $44 \%$ of total nursing staff) rated patients due to random selection of the patients. Consequently 13 nurses did not rate any patients. The ethnicity of those staff who did ratings was; New Zealander/Pakeha represented $59 \%(n=25)$ of the participants, while $26 \% \quad(n=11)$ identified themselves as having European (German, Irish and British) ethnicity. 'Other' staff participants made up $14 \%(n=6)$ of the group which included Indian, Philippines and South African ethnicity. Of the 13 nurses who did not participate in ratings their ethnicity was; New Zealand/Pakeha represented $61 \%$, while $31 \%$ were European and $8 \%$ included 'Other" ethnicity as defined above. Table 8 summarises the demographic detail of the staff participants who completed ratings.

Table 8. Demographic Detail of Staff Participants

\begin{tabular}{|l|c|c|}
\hline & Number & Percentage \\
\hline Staff & & \\
Nurses & 35 & $44 \%$ \\
Doctors & 7 & $58 \%$ \\
\hline Ethnicity & & \\
New Zealand/ Pakeha & 25 & $59 \%$ \\
European & 11 & $26 \%$ \\
Other & 6 & $14 \%$ \\
\hline
\end{tabular}

The levels of experience of nursing participants were categorised in two different ways. Firstly by the C\&CDHB Clinical Career Pathway (CCP) from RN2a to RN4. Secondly by years worked in C\&CDHB ICU along with years worked in any ICU. This was done to allow comparison with previous reliability studies of sedationscoring tools and to allow for comparisons with future studies. During the time of this research the ICU had two RN4 level staff nurses on the C\&CDHB Clinical 
Career Pathway (CCP), 35 at RN3, 26 at RN2b and 17 at RN2a. Riker et al. (1999) used the time limit of three years to define experienced ICU nurses, for that reason I have included demographic data of the participants in a similar fashion. Table 9 presents the clinical experience of the staff. The mean number of years nursing participants had worked in Wellington ICU was 3.5 years (range $1-17$, sd 3.5 years). Of the 13 nurses who did not do ratings their mean number of years worked in Wellington ICU was 4.0 years (range 1-10, sd 2.6 years). Sixty six percent of nursing staff had worked in Wellington ICU for 3 years or less, then again many staff had worked in ICUs elsewhere as indicated by only $31 \%$ of nursing staff having worked in any ICU for three years or less. In comparison the mean number of years nurses worked in any ICU was 6.9 years (range 1-18, sd 5.0 years). This indicates that a significant number of the staff participants had previously worked in other ICUs nationally and internationally (Table 9).

Table 9. Experience of Staff Participants who Completed Ratings

\begin{tabular}{|l|c|c|c|c|c|}
\hline \multicolumn{1}{|c|}{$\begin{array}{c}\text { Staff } \\
\text { category }\end{array}$} & No. & $\begin{array}{c}\text { Years in } \\
\text { any ICU }\end{array}$ & $\begin{array}{c}\text { Years in } \\
\text { Wellington } \\
\text { ICU }\end{array}$ & $\begin{array}{c}\text { ICU } \\
\text { Specialty } \\
\text { Course }\end{array}$ & $\begin{array}{c}\text { Previous Experience } \\
\text { with Sedation-Scoring } \\
\text { Tool } \\
\text { Yes/No }\end{array}$ \\
\hline Consultant & 4 & $6-12$ & $1-12$ & NA & 0 \\
Registrar & 3 & $\leq 1$ & $\leq 1$ & NA & 0 \\
\hline Total & 7 & & & & 5 \\
\hline RN2a Nurse & 8 & $1-14$ & $1-14$ & 4 & 2 \\
RN2b Nurse & 10 & $2-18$ & $1-7$ & 7 & 7 \\
RN3 Nurse & 15 & $1-18$ & $1-17$ & 10 & 0 \\
RN4 Nurse & 2 & $4-5$ & $4-5$ & 2 & $\mathbf{1 4}$ \\
\hline Total & 35 & & & 23 & \\
\hline
\end{tabular}

${ }^{*}$ New Zealand Post-graduate Certificate and United Kingdom Specialty Critical Care Course.

Of the 13 nurses who had not done ratings $46 \%$ had worked in Wellington ICU for three years or less, while $31 \%$ had worked in any ICU for less than three years. For this group of nurses the mean number of years worked in any ICU was 6.0 (range 1-13, sd 3.7 years). Forty nine percent of nursing participants also said that they held a New Zealand post-graduate certificate in the specialty of intensive care. In comparison $34 \%$ held no formal ICU specialty course and 
$17 \%$ held an international specialty intensive care course. Forty six percent of those nurses who did not do ratings held a New Zealand post-graduate certificate and $8 \%$ held an international specialty course.

All staff participants were also asked if they had previous experience with sedation-scoring tools. Of the $14(29 \%)$ who answered yes, all were nurses. None of these nurses had previously used the SAS prior to working in Wellington ICU. The sedation-scoring tools that some nursing staff had experienced were the Ramsay Scale, the Cook Sedation Score, Waikato ICU Sedation-Scoring tool, the modified Glasgow Coma Scale, the Bloomsbury Sedation Scale and two were tools developed for the specific ICUs staff had previously worked in. Only one nurse who did not do ratings had previous experience with a sedationscoring tool.

The medical staff experience varied significantly (Table 9). For medical staff working in Wellington ICU the mean number of years was 3.3 years (range $\leq 1$ 12 , sd 4.1 years) and $71 \%$ had worked there for 3 years or less. This was influenced by the 6-month rotation of registrars through the ICU and two consultants who had worked in the unit two years or less. In comparison the mean years worked in any ICU by medical staff was 5.0 years (range $\leq 1-12$, sd 4.2 years) and $43 \%$ of medical staff participants had worked in any ICU for three years or less; these were all registrars. The consultants ranged from six to twelve years experience in intensive care medicine. The registrars had only three months experience with the SAS compared with the nursing staff that had been using it in their practice for seven months.

\section{The Patient Population}

Of the 70 patients randomly selected only one was excluded because they had been evaluated some days earlier on a previous admission, leaving 69 patient evaluations. The sample included $35 \%$ female $(n=24)$ and $65 \%$ male $(n=45)$. The mean age was 58 years (range 17-83, sd 18.3 years). The demographic characteristics of the patient population are presented in Table 10. 
Patient ethnicity was collected from the C\&CDHB patient admission form. This sheet is completed by all patients or their support persons on admission to hospital. Patient ethnicity data indicated that $13 \%(n=9)$ of the patients were Maori, $73 \%(n=50)$ were Pakeha (New Zealander)/European, 4\% $(n=3)$ were Pacific Island and 10\% ( $n=7)$ were of 'Other' ethnicity which included Chinese, Indian, Greek, and British.

\section{Table 10. Demographic Characteristics of Patients Being Assessed using the Sedation-Agitation Scale}

\begin{tabular}{|l|c|c|}
\hline \multicolumn{1}{|c|}{ Demographic Feature } & Number & Study Population \\
\hline Number of Patients & & 69 \\
\hline Gender: & & \\
Male & 45 & $65 \%$ \\
Female & 24 & $35 \%$ \\
\hline Age (years) & Range $17-83$ & Mean 58 years \\
\hline Ethnicity: & 50 & $73 \%$ \\
Pakeha/European & 9 & $13 \%$ \\
Maori & 3 & $4 \%$ \\
Pacific Island & 7 & $10 \%$ \\
Other & \\
\hline
\end{tabular}

* Other includes Chinese (3), Indian (1), Greek (1) and British (2)

Table 11 represents the clinical features of the patient sample. The patient length of stay (LOS) in the ICU at the time of the ratings, for the 69 people ranged from 0-19 days, with a mean of 2 days (sd 3.4) and a median of 1 day. Intubated patients made up $91 \%(n=63)$ of the patients in the study, while $9 \%(n=6)$ were extubated. Two patient diagnostic groups (according to the APACHE II codes) made up over $50 \%$ of the admissions; cardiac surgery $33 \%(n=23)$ and trauma $20 \%(n=14)$. The APACHE II scores ranged from 4-43 with a mean score of 25. There were however nine patients (who were not surgical cardiothoracic cases) with no APACHE II scores because they were in the ICU less than 24 hours. These patients either died soon after admission, were transferred to a ward following a short period of close monitoring (usually post-anaesthetic) or were discharged home. It is very unusual to discharge a patient home directly from an $I C U$, it occurs occasionally in cases of recreational drug overdoses. The Tu 
scores for the 21 cardiothoracic surgical patients ranged from 1-4 with a mean score of 2.

Table 11. Clinical Features of Patients Being Assessed using the SedationAgitation Scale

\begin{tabular}{|l|c|c|}
\hline \multicolumn{1}{|c|}{ Demographic Feature } & Number & Study Population \\
\hline \multicolumn{1}{|c|}{ Length of Stay (days) } & & Range 0-19 \\
\hline \multicolumn{1}{|c|}{ Yo } & 63 & $91 \%$ \\
\hline Intubated & 6 & $9 \%$ \\
\hline APACHE II Codes & 5 & $7 \%$ \\
Cardiac non-surgical & 23 & $33 \%$ \\
Cardiac surgical & 2 & $3 \%$ \\
Neurological non-surgical & 6 & $9 \%$ \\
Neurological surgical & 8 & $12 \%$ \\
Other & 4 & $6 \%$ \\
Respiratory non-surgical & 3 & $4 \%$ \\
Respiratory surgical & 4 & $6 \%$ \\
Sepsis & 14 & $20 \%$ \\
Trauma & & \\
\hline APACHE II Scores ${ }^{*}$ (n=39) & 3 & \\
$<10$ & 12 & \\
$10-20$ & 11 & \\
$21-30$ & 10 & \\
$31-40$ & 3 & \\
$>40$ & & \\
\hline TU Scores (n= 21) & 15 & \\
$\leq 1$ & 2 & \\
$2-3$ & 0 & \\
$4-6$ & 4 & \\
$\geq 7$ & & \\
\hline
\end{tabular}

${ }^{*}$ Other includes overdose (4), gastro non-surgical (1) and gastro-surgical (3)

\# Nine cases with no APACHE II Scores, as in ICU < 24 hours.

\section{Clinical Features of Sedation in Wellington}

Paralysing agents, such as pancuronium or rocuronium, had not been given to any of the patients selected for this study. Patients receiving bolus sedatives made up $12 \%(n=8)$ of the sample while $88 \% \quad(n=61)$ of patients received sedative infusions. Of the eight patients who received bolus sedation, the most common drug given was Midazolam $(n=5)$, followed by Propofol $(n=2)$ and Thiopentone $(n=1)$. Table 12 provides details of the drugs and route given. In patients who received a continuous infusion of a sedative, Propofol was given 
$79 \%(n=48)$ of the time and Midazolam 21\% (n=13) of the time. This study did not record instances of bolus sedation being given on top of a continuous infusion.

Table 12. Sedatives used by Sample from August - September 2003

\begin{tabular}{|l|c|c|}
\hline & Number & Percent \\
\hline Type of Sedation & 8 & $12 \%$ \\
Bolus & 61 & $88 \%$ \\
Infusion & 5 & $63 \%$ \\
\hline Bolus Drug $^{*}$ & 2 & $25 \%$ \\
Midazolam & 1 & $12 \%$ \\
Propofol & & \\
Thiopentone & 13 & $21 \%$ \\
\hline Infusion Drug & 48 & $79 \%$ \\
Midazolam & 0 & \\
Propofol & $2 \%$ & \\
Thiopentone & & \\
\hline
\end{tabular}

* This does not identify situations where a patient was given a bolus on top of a continuous infusion.

\section{Nurse-Doctor Sedation-Scoring in Wellington}

There was no set pairing of doctors and nurses, the nurse-doctor pair was determined on the day by who was caring for the randomly selected patient. In all cases the primary nurse caring for the patient was consistently the individual performing the assessment. The number of ratings each individual nurse did as part of this study ranged from 1-6 (mean 1.2, sd 1.1). Given the difference between the number of nurses and doctors involved in this study it is not surprising that the number of ratings medical staff performed ranged from 1-19 (mean 9.9, sd 5.0).

Table 13. Numbers of Agreement by Sedation-Agitation Scale

\begin{tabular}{|c|c|}
\hline SAS Score & Number of Ratings the Same \\
\hline 1 & 23 \\
\hline 2 & 7 \\
\hline 3 & 7 \\
\hline 4 & 13 \\
\hline 5 & 1 \\
\hline
\end{tabular}


The ratings were not normally distributed; most patients' scores were clustered around the SAS scores of 1 and 4 (Table 13). No patients were identified at the extreme end of the SAS score (at 6 or 7 ) for agitation and very few patients were identified at a score of $5(n=3)$ (anxious or mildly agitated). Although the doctors used a range of scores they were more likely to use the SAS of 1 and 4 whereas the nurses' scoring did not follow such a pattern and they used a wider range of scores from 1 through to 5 , as can be seen from Figure 8 .

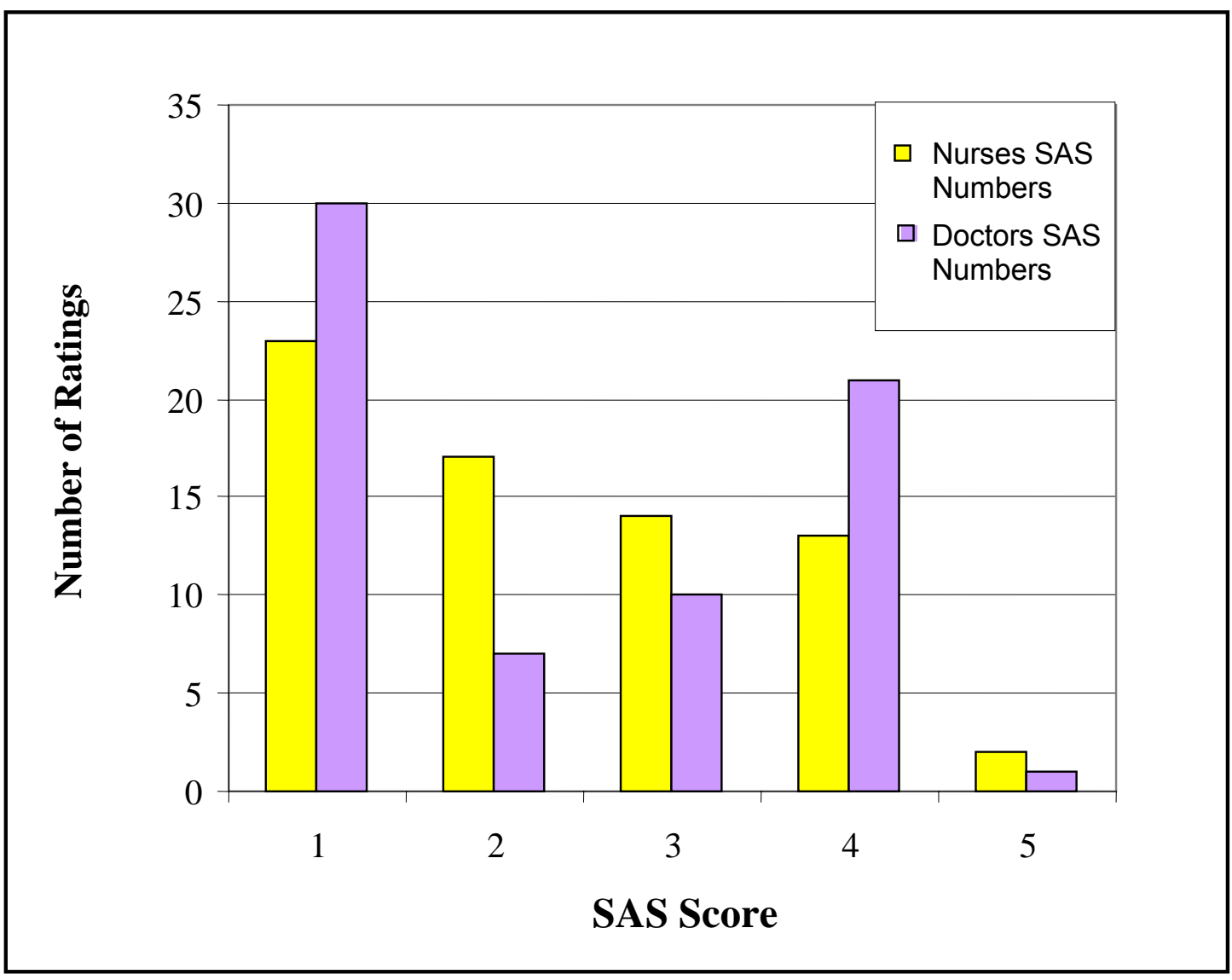

Figure 8. Nurses and Doctors Sedation-Agitation Scale Ratings

\section{The Reliability of the Sedation-Agitation Scale in Wellington}

The doctor and nurse raters selected the same scores in $74 \% \quad(n=51)$ of the ratings. The majority of paired agreement ratings were around the level $1 \quad(n=23)$ and $4(n=13)$ (Table 13). In $27 \%$ of cases there was no agreement between the raters (see Table 14). As a measure of reliability the mean SAS score recorded for both the nurses and doctors are similar $(2.33 \& 2.36$, sd $1.21 \& 1.35$ 
respectively). Within the Wellington ICU the results of the weighted kappa (K) statistic, $\mathrm{K}=0.82$, indicating very good agreement (reliability) according to Altman (1991).

\section{The Difference in Agreement}

In considering where the difference (disagreement) was between the nurse and doctor ratings it is apparent from Table 14 that the ratings with disagreement were only one level of the SAS away from each other. In 10 paired ratings, the nurse gave a patient a SAS score one level lower than the doctor. In eight situations, the nurse rated the patient one level higher than the doctor on the SAS.

Table 14. Difference between Nurses and Doctors Ratings

\begin{tabular}{|l|c|c|}
\hline \multicolumn{1}{|c|}{ SAS score difference } & $\begin{array}{c}\text { No. of paired } \\
\text { ratings }\end{array}$ & Percentage \\
\hline $\begin{array}{l}\text { Nurse score lower by } \\
\text { one level }\end{array}$ & 10 & $15 \%$ \\
\hline Ratings equal & 51 & $74 \%$ \\
\hline $\begin{array}{l}\text { Nurse score higher by } \\
\text { one level }\end{array}$ & 8 & $12 \%$ \\
\hline
\end{tabular}

Further analysis of this difference was undertaken to establish if there was a pattern to this difference and whether the difference was attributable to a nurse or doctor difference; furthermore whether this could be attributable to a particular patient diagnostic group, ethnicity or gender. It did not appear that a nurse or doctor difference would explain the differences in the ratings as the differences occurred in both directions of the SAS scoring tool. On further investigation to see if those staff that did three or more ratings was the source of the difference I found that this was not the case. No specific variable was identified that would explain the difference with the exception of considering if the disagreement was around a specific SAS score. However a pattern was found to this disagreement. Table 15 illustrates the direction of the difference between the SAS scores. 
Table 15. The Direction of Difference between the Nurses and Doctors using the Sedation-Agitation Scale

\begin{tabular}{|c|c|c|}
\hline Paired SAS Score & $\begin{array}{c}\text { Nurse Score Higher- } \\
\text { Doctor Score Lower } \\
\text { (No.) }\end{array}$ & $\begin{array}{c}\text { Doctor Score Higher- } \\
\text { Nurse Score Lower } \\
\text { (No.) }\end{array}$ \\
\hline $1-2$ & 7 & 2 \\
\hline $2-3$ & & 8 \\
\hline $3-4$ & 1 & \\
\hline $4-5$ & 1 & \\
\hline
\end{tabular}

This direction of the difference does not mean either the nurses' or doctors' scores are right or wrong, only that the difference was found around a particular SAS score and the direction of the difference was consistent for these scores. For the most part the difference between the scores centred on SAS score 3-4 and the SAS scores 1-2. The difference between a score of 1 compared with 2 is that a 1 refers to a patient with minimal or no response to stimuli who does not communicate or follow commands. A score of 2 refers to a patient who arouses to physical stimuli, may move spontaneously but who does not communicate or follow commands. The direction of the difference for the SAS score of 1-2 was the nurses consistently scoring the higher (that is 2); whilst with the difference with the SAS score 3-4, the doctors consistently scored the higher of the two scores (that is 4). A score of 3 is given to a patient who is difficult to arouse, will awaken to verbal stimulation or gentle shaking, may follow a simple command but drifts off again. Whereas a score of 4 is given to a patient who is calm, awakens without difficulty and follows instructions.

Consideration was also given to specific patient variables to see whether there was anything about the patients that was impacting on the SAS scoring (Table 16). Paired sample t-tests were used on age ( $\mathrm{t} 1.74$, df 67, $p=0.087$ ) and length of stay ( $\mathrm{t}-1.220$, df $67, p=0.227)$. This indicates that there was not a statistically significant difference in the age of patients and length of stay in the group where the SAS scores were equal between the raters and the group where there was disagreement between the raters. 
The chi-square test for independence was used on the variables of gender, intubation, mode of sedation and cardiac patients. These variables were compared in both the group with equal SAS ratings and the group with non-equal SAS ratings. None of these relationships were found to be statistically significant. No other variables could be tested as their cell sizes were less than 5 .

Table 16. Patient Variables Impact on Ratings

\begin{tabular}{|c|c|c|}
\hline Variable & Equal (n=51) & Non-equal (n=18) \\
\hline Age & $\begin{array}{c}59.9 \\
\mathrm{sd}(17.0)\end{array}$ & $\begin{array}{c}51.2 \\
\text { sd (21.0) }\end{array}$ \\
\hline $\begin{array}{c}1.69 \\
\text { Ld (2.8) }\end{array}$ & $\begin{array}{c}2.83 \\
\text { sd (4.8) }\end{array}$ \\
\hline $\begin{array}{c}\text { Gender of Stay } \\
\text { Male }\end{array}$ & $73 \%(33)$ & $27 \%(12)$ \\
Female & $75 \%(18)$ & $25 \%(6)$ \\
\hline $\begin{array}{c}\text { Intubated } \\
\text { Extubated }\end{array}$ & $71 \%(45)$ & $29 \%(18)$ \\
\hline $\begin{array}{c}\text { Mode of Sedation } \\
\text { Bolus } \\
\text { Infusion }\end{array}$ & $100 \%(6)$ & $0 \%(0)$ \\
\hline $\begin{array}{c}\text { Diagnostic Code } \\
\text { Cardiothoracic }\end{array}$ & $75 \%(6)$ & $25 \%(2)$ \\
$\begin{array}{c}\text { Surgery } \\
\text { Other }\end{array}$ & $74 \%(45)$ & $26 \%(16)$ \\
\hline
\end{tabular}

\section{Conclusion}

Sixty nine adult ICU patients and $53 \%$ of staff participated in this reliability study. The majority of patients were sedated with continuous infusions of Propofol. The experience of the staff participants in the ICU varied with $31 \%$ of nursing staff and $43 \%$ of medical staff having worked in any ICU for three years or less. Medical staff, because of their smaller numbers, performed more ratings than the nursing staff. The doctor-nurse paired ratings were the same in $74 \%$ of cases. Where there was disagreement, it was only one level of the SAS away from each other. Further examination of the difference noted the majority were centred on a 
SAS score of either 1-2 or 3-4. No other variables were identified as having an impact on the SAS ratings.

The following chapter considers these results in detail and their implications for this research, future research and clinical practice. Recommendations are made for further research and changes in clinical practice within Wellington ICU. Finally the chapter concludes with a reflection on the limitations of this study, the SAS and a reflection on the research process. 


\section{Chapter 7- Discussion}

This study provides further confirmation of the inter-rater reliability of the Sedation-Agitation Scale (SAS) in adult Intensive Care Unit (ICU) patients. In earlier studies experienced ICU nurses (Riker et al., 1999) or staff that were unfamiliar with the SAS (Brandl et al., 2001) were participants and the tool was compared with other sedation-scoring tools to test for validity. In this research the weighted Kappa (к) score compares favourably with both studies. This study, performed in a natural setting, has reported a $\mathrm{k}$ score of 0.82 whereas Riker et al. delivered $\mathrm{k}=0.92$ (experienced nurses) and Brandl et al. $a \mathrm{k}=0.85$ and 0.87 (first exposure bed-side nurses). In each study there was variation in the staff and patient population and the research method.

This study answers the research question and null hypothesis which it intended to consider. Nurses and doctors in the Wellington ICU do rate the SAS similarly and there is little difference in the ratings by the nurses and doctors. Whilst this study could not technically be classified as an inter-rater reliability study it does confirm the reliability of the SAS by demonstrating that nurses and doctors working in intensive care are able to use the tool reliably and have a similar understanding of its meaning and context for individual patients, this is despite the tool being designed and initially tested in the United States of America (USA). Nevertheless it is still relevant to the New Zealand culture and context. This study confirms that independent raters can reliably quantify ICU patient behaviour by using the SAS. This study aimed to look closely at the consistency of the ratings made by different raters and the proportion of time raters agreed on a specific level of the SAS. This chapter considers the results presented, reflects on the research experience and makes recommendations for clinical practice and future research.

This reliability study is different from earlier evaluations of the SAS in several respects. Firstly the researcher did not attempt to validate the SAS by comparing it with any other sedation-scoring tool. The SAS was already part of the practice 
in the ICU and this research was being performed in a natural general ICU setting. The researcher saw no need to repeat research that had been done so comprehensively previously and introducing an alternative sedation-scoring tool may have created confusion in the clinical setting. Secondly, all staff in this study had some experience with the SAS as part of their daily practice. No member of staff participated that had not received training on the SAS. In this research the nurse was consistently the staff member who performed the evaluation while the doctor observed the patient. To my knowledge using the patient's own nurse was the first time this had been done using the SAS in a research project. Finally the aim of this study was to differentiate the raters (nurse and doctor) to determine whether they rate similarly using the SAS. In the previous research the raters were not defined by their profession/role. Although this study followed a similar design to that of Riker et al. (1999) and Brandl et al. (2001), there was one key difference. Both Riker et al. and Brandl et al. used a convenience sample of patients whilst in this study the patients were randomly selected to ensure a heterogenous ICU patient population were rated.

\section{The Staff and Patient Sample}

Although 48 nurses had consented to participate in this study, 13 nurses did not do ratings on patients because of how patients were randomly selected. Further demographic analysis of this group of nurses was undertaken to determine if they were a different group to the nurses that did complete ratings. This analysis indicated that these 13 nurses were not dissimilar in demographic detail to those nurses who had undertaken ratings. Further comparison of the staff participants with staff that had not consented to participate was not possible as specific demographic data of the non-participants (except nursing CCP level) is not available at Wellington Hospital.

Wellington ICU has a significant number of nursing staff from other countries which are indicated in the ethnicity of the sample. When considering the experience of the nursing staff, many have experience in other ICUs worldwide. This appears to reflect a growing trend in ICUs; in view of the fact that there is an 
international nursing shortage many ICUs have recruited staff internationally. Sixty six percent of nursing staff had worked in Wellington ICU for three years or less, on the other hand, when taking into consideration nursing staffs' experience in other ICUs only $31 \%$ had worked in any ICU for three years or less. The mean number of years the nurse raters worked in Wellington ICU was 3.5 (sd 3.5) in comparison with that found by Riker and colleagues (1999) 5.9 years. However, Riker et al. had purposely selected raters with greater than three years experience in their ICU. The mean number of years nursing participants in this study had worked in any ICU was 6.9 (sd 5.0). These data were not reported in Brandl et al.'s (2001) study.

In comparison the medical staff experience varied considerably. The $71 \%$ of medical staff who had worked in the Wellington ICU for 3 years or less was influenced by the 6-month rotation of registrars through the ICU. The registrar participants had not previously worked in an ICU and had only been familiar with the SAS for 3 months prior to the commencement of this study. Medical staff in the ICU are likely to be less familiar with the SAS as they are not evaluating patients as regularly as the nursing staff. This may have also had some bearing on the disagreement between the raters (discussed later in the chapter). The number of ratings performed by medical staff was 1-19 compared with 1-6 for nursing staff. This is not unexpected considering the number of medical staff in the study totals 7 , whereas there were 35 nursing staff. This difference was not thought to impact on ratings because there was little warning for staff (in particular medical staff) therefore they had little time to prepare for the ratings.

One of the strongest points of this study is that the study context mimics the clinical reality common in many ICUs. It was important that the study was performed in a natural setting in order for the results to be generalisable to the larger ICU population. This in part indicates the reason for not excluding patients who could possibly die within 24 hours or those with neurological impairment, as had been the case with previous studies (Riker et al., 1999; Brandl et al., 2001). A minimum number of exclusion criteria were involved in order to present a 
heterogenous sample that reflected the natural ICU setting. In comparing the patient demographics with the demographic details of patients collated annually by the Wellington ICU there were some similarities and differences with the sample.

For the 2002-2003 financial year in the Wellington ICU, the percentage of female patients was $34 \%$, with $66 \%$ of males. This study produced a sample of $65 \%$ males and $35 \%$ females. The mean age of the study patient sample was 58 years (range 17-83) compared to the ICU figures of a mean of 57 years (range 090 years). Cardiothoracic surgical cases made up $40 \%$ of the total admissions to the ICU compared with $33 \%$ in this study. These demographic details indicate that the study patient sample was similar in many regards to that of the ICU population for the year July 2002 to June 2003. The mean length of stay in the 2002-3 year was 24 hours (range 0-201 days) whilst in this study it was two days (range 0-19). Many patients in the study were scored between day zero and one. The range of days was possibly influenced because patients could only be rated once. Ninety one percent of the patient sample were intubated and ventilated at the time of the ratings. This may also be a reflection of being evaluated on day zero or day one as it reflects the stage of their ICU care. The APACHE II scores and the Tu scores indicate the spectrum of severity of illness, from high dependency requirements to critically ill with limited chance of survival.

A larger patient sample would have allowed for further analysis of patient variables, to ascertain whether there was something about the patients that was impacting on the scoring. Because the cell size was too small for analysis of other patient variables, such as other diagnostic codes, mode of sedation, extubated patients and ethnicity, a larger study could be undertaken to ensure sufficient numbers for further analysis of these factors.

\section{The Ratings}

The ratings in this study are not normally distributed. No patients were identified at the extreme end of the SAS (at 6 or 7). This is possibly because in the 
Wellington ICU patients who are significantly agitated are assessed and managed promptly. It would have been very difficult to obtain those scores as the agitation was occurring, unless the timing of a rating and the presence of the researcher coincided with the agitated behaviour being exhibited. Therefore it is problematic to assess the reliability of rating patients at the extreme ends of the SAS because of a lack of patient numbers; it would require a very large patient sample and possibly a complex research method to achieve this. Riker and colleagues (1999) went to some length to achieve this.

It is not unexpected that there were many scores of four as this is often a common predefined goal of therapy. Nevertheless this study presented a significant number of ratings with a score of one. There are two reasons why this may have occurred. Firstly many of the ratings were performed on patients who were day zero or day one in the ICU. A SAS score of one may reflect the context of their illness and physiological instability requiring the patient to be heavily sedated or a patient who has not yet cleared heavy anaesthetics postoperatively. Finally, this may indicate a need for further education and training including a discussion of the levels of the SAS.

Another possible explanation for the number of SAS scores of one where there was disagreement in the ratings may be to some degree explained by the difference in the professions of the raters and their roles in ICU. As discussed in Chapter Three, how nurses and doctors assess a patient may be influenced by the weighting they give certain behavioural characteristics. Raters can also be influenced by environmental, philosophical and contextual factors (Benner, 1984; Bucknall, 2000, 2003) which Chapter Two explored in detail. Whilst a significant amount of research about influences on clinical decision-making has been undertaken with nurses it cannot be said that the same influences do not have a similar impact on medical staff. What is known about the Wellington ICU context is that the nurse is at the patients' bedside almost continuously, monitoring and assessing their physiological and behavioural indicators. In comparison, there is 
only one consultant and two registrars on each shift, therefore they are not able to give all their attention to one patient and must prioritise the needs of all.

In taking all this into consideration and from personal experience I would argue that the nurses use more than the behavioural cues given by the patient at the time of the assessment in determining their SAS score. Although behavioural cues may hold the most weighting other contextual influences may also impact on the decision made. For example, if a nurse caring for a patient has been trying to manage agitation all day (a SAS of 6), but at the time of assessment the patient is calm and co-operative (a SAS of 4 ) this prior behaviour may have some influence on the score that the nurse gives the patient. Another example would be a patient that regularly becomes agitated during suctioning or turning but is otherwise calm, may be more likely to be considered agitated as part of a global assessment. Medical staff may not be aware of the issues the nurse has been concerned with because they have not been present at the bedside for some time and have not observed them. These same issues were also identified by Riker et al. (1999). In this research where a nurse had scored a patient a two and the doctor had scored a one, this may have been influenced by the nurse having noticed subtle behavioural changes over a period of time where the patient was being aroused by physical stimulus, which was not present at the time of a rating. Does this make the nurses rating wrong or right? Or is their decision more informed by knowing the patient more? The same should be considered for the difference between the SAS 3-4. Is this difference influenced by the length of time each professional has with the individual patient getting to know them? Further exploration of these differences by doctors and nurses on this and other patient assessment tools is required.

The goal of sedation for individual critically ill patients varies from patient to patient and within individual patients over time. Sedated patients are not usually able to communicate effectively with their nurses to assist in determining an adequate level of sedation. Therefore understanding a patient's unstable condition and anticipating complications is inextricably linked with knowing the 
patient (Benner et al., 1999). This fundamental concept of knowing or being with a patient highlights a key difference between the nursing and medical profession, furthermore it may influence how nurses score patients using a sedation-scoring tool.

Regardless of the growing evidence supporting the use of sedation scales along with guidelines, general acceptance into clinical practice remains a challenge. Many sedation-scoring tools have not been published with guidelines or educational supports which would aid to understand their appropriate use. The SAS was one of the few that did and this education programme was improved by further communications with one of its creators. In addition there may also be a perception in some ICUs that prescriber autonomy may be compromised if nurses are given more scope in their practice in managing the sedation needs of their patients, which could further hinder sedation-scoring tools implementation. Devlin et al. (2001) suggest that facilitating clinical judgment to guide the choices of sedation therapy (as opposed to dictate) maintains autonomy for all professionals and improves the likelihood of a tool's acceptance in clinical practice.

Despite the difference in clinical experience of nursing and medical staff in this study there was still significant agreement between the nurse-doctor observations. I believe this was influenced by the education and training on the SAS that all staff in the ICU had received. This was initially stressed by Riker et al. (1999), then subsequently by Brandl and colleagues (2001). It is crucial that all staff in ICU receive formal training on a sedation-scoring tool to ensure there is consistency in the use of the tool, consistency in the quality of sedation given, and to ensure that communication between nurses and physicians about the effectiveness of sedation regimens is effective. Since the data collection phase of this study has been completed I have been asked to give several additional education sessions on sedation assessment in ICU. The feedback from these sessions suggests that regular reviews and ongoing education is also important to remind staff of the key points in using the SAS. Therefore I would recommend 
that revisiting the sedation-scoring education programme on a regular basis may assist to ensure consistency remains when evaluating patients with a sedationscoring tool. Further training should involve doing some paired ratings and then discussing as a group why the individuals chose the scores they did. This may assist with further clarification of the levels of the tool and talking around the difference may provide more consistency of ratings in the future.

One further point to consider is that in this research it was the nurse who always performed the procedures required for the SAS assessment. It is difficult to say categorically whether this had an impact on the ratings, however the researcher believes this had little impact as where there were instances of disagreement it was in both directions. The decision was made to have the nurse do the procedures because in the natural ICU setting they would be the professional at the bedside evaluating the patient the majority of the times and titrating sedatives accordingly.

No other specific features of the staff or patients were identified that may have explained the disagreement in the ratings. This was in part due to small cell sizes limiting further analysis. Future studies with larger sample populations may be able to investigate associations between variables and differences in ratings.

An important question raised from this research is, is whether the disagreement between the raters is clinically significant? It is not within the scope of this study to answer this question because the actions/decisions of the nurse and doctor following their ratings were not recorded or considered. It was not the intention of the research to document their decision-making based on their assessment as the SAS tool was not to be used as a basis for clinical decision-making until its reliability had been confirmed. However, from what I have observed in clinical practice it is unlikely that a score difference of one would make a major impact on the management of an individual patient as other factors are included in sedation management, such as patient cardiovascular stability. I believe in the natural setting where there is disagreement the raters would consider their assessment 
jointly, and then as a result of their discussions they would be more likely to make a joint decision on what steps to take.

\section{The Sedation-Agitation Scale}

Further analysis of the research data indicated which scores were subject to the most disagreement. The reason for considering the difference in the scores was to understand the factors that may cause raters to disagree, with an ultimate goal of improving their consistency and accuracy. The difference in scores occurs because in the mind of the rater, the weight of evidence of sedation-agitation on the whole is an aggregate made up of various behavioural characteristics and the weighting attached to each feature. Raters may vary in terms of which features they notice and the weights they associate with each; ultimately this will influence what score they give a patient. In this study the difference in the ratings was never more than one level away from each other and the majority appeared to be around the SAS score of 1-2 and 3-4. The direction of the difference was consistent in each account. Where there was a difference between the SAS of one and two, the nurse would consistently score the two whilst the doctor the one. With the SAS scores of 3-4, the doctor would consistently score the four whilst the nurse would score the three. There are several points to be considered around this issue.

There may need to be additional consideration and refinement of the language of the SAS. There is conceivably something around the language used that does not clearly define the levels of the scoring tool. In considering the descriptive behaviours of a SAS of one and two, I believe their descriptions are very similar and have recently caused some confusion and discussion within the Wellington ICU. The term 'minimal' within a SAS of one may be problematic. It can be interpreted as making some response to noxious stimuli, however subtle it may be, and this description of behaviour is also indicated in a SAS of two (see Appendix A). The key terms which define the difference between the levels one and two from three and above is awakens (level three and above) and arouses (level two and below). It was not until the researcher had personal 
communication with Dr. Riker (R.R. Riker, personal communication, April 16th, 2003) that I became aware of the importance of clearly defining these levels and providing further guidelines for the ICU staff. My recommendation is that the term 'minimal' be removed from level one as it implies that there is some response which is contradictory to the level one characteristic of unrousable.

There may also be some confusion surrounding the descriptive language used in the SAS levels three and four. In level three both terms (arouse and awaken) are used to describe the expected behaviour. I would recommend that the term arouse is removed from level three of the SAS, so that descriptors read as "difficult to awaken, but does awaken to verbal stimuli or gentle shaking, follows simple commands, but drifts off again" (see Appendix A). In this way there is a clear separation between level two and below (defining arouse) and level three and above (defining awaken). In the context of ICU sedation each of these terms has a different meaning to their dictionary definition hence they require further clarification. Awakening is most likely inaccurate in the ICU setting because most patients do not display normal sleep patterns (Kress et al., 2002). Awakening denotes an emergence of consciousness. Whereas arouse refers to a state of acute mental status change where the patient is brought to the brink of consciousness. This patient may or may not be able to follow a simple command but quickly returns to an unconscious state. These points clearly support Egerod's (2002) contention that sedation terminology can be ambiguous and have an impact on clinical decision-making. It is essential that it is appraised, defined and be included in future critical care curriculum. Patients may repeatedly be inappropriately sedated because of a lack of recognition of oversedation and understanding of the assessment tool used, in addition to inadequate communication between the professions.

During the introduction of the SAS into Wellington ICU (prior to the research) staff made recommendations for further clarification of the levels of the SAS. Several staff suggested that at level 6 of the SAS, where it describes the use of physical restraints, there should also be mention made of the use of chemical 
restraints (that is pharmacological restraints). In clinical practice, medications (for example diazepam and haloperidol) are used at times to restrain patients who are a danger to themselves. The context of physical restraints needs to be considered when assessing a patient. Some staff may use physical restraints prophylactically, anticipating agitation, but if at the time of an assessment the patient is calm and co-operative (level 4), a patient should not necessarily be assessed as a level 6 because of the presence of restraints. One final observation made by the staff in the Wellington ICU was that patients who are calm and co-operative (level 4) may also touch their invasive catheters (such as an endotracheal tube) but are not necessarily going to attempt to remove them. As patients regain consciousness they become curious of their surroundings and will often explore them and are able to process that these catheters are important and not to be removed. This type of patient would still be classified as a score of four.

\section{The Sedatives}

The significant majority of patients receiving continuous infusions (88\%) of sedatives were on Propofol infusions (79\%). Only a small amount of sedatives was given as a bolus during the study (12\%). Further auditing of the types and mode of sedatives is required in this ICU to determine whether this is an appropriate use of sedatives for individual patients.

It is essential that an appropriate goal for sedation is determined for individual patients. It should be clarified at the beginning of ICU care and reassessed on a regular basis as the patient's clinical condition changes. Sedation regimes should be prescribed with appropriate flexibility to allow titration of sedatives to the desired goal. This may help to reduce the tendency of staff to 'lock in' to large sedative infusion rates, which although may be appropriate on day one of ICU, possibly will not be needed on subsequent days (Kress et al., 2002). Wellington ICUs current practice involves running a sedative infusion in combination with an analgesic. This infusion is routinely morphine and midazolam $1 \mathrm{mg} / \mathrm{ml}$ in combination. Both drugs are for different purposes. Whereas a patient may have 
an ongoing analgesic requirement at, for example $8 \mathrm{mg} / \mathrm{hour}$, their sedative requirement may be less than that. Nurses are unlikely to compromise a patient's analgesic requirement therefore these infusions tend to remain 'locked in' at a higher rate. On reflection of this practice in Wellington ICU I would recommend that these medication infusions be split so they can both be titrated appropriately for the individual needs of the patient and a sedation and analgesic guideline be developed to assist staff in titrating these medications appropriately.

\section{Limitations of the Study and the Sedation-Agitation Scale}

There are several potential limitations of the SAS. First the SAS relies on patient auditory acuity and an understanding of the English language. In the multicultural society we now find ourselves, it is not uncommon to come across patients with little comprehension of the English language. The SAS is also not suitable for patients with a hearing impairment or those with a degree of paresis which prevents an expected response. It remains unclear whether the SAS is appropriate in any patient with a neurological impairment. In reliability testing, the researcher acknowledges that there were very few patients for whom agitation was established and where there was agitation recognised there was not complete agreement between the raters. Finally, responsiveness or the degree to which the tool can identify significant changes in sedation over time was not tested. Responsiveness has not yet, to my knowledge, been tested for any sedation scale in an ICU population (De Jonghe et al., 2000).

There are several limitations to this study. As has been noted, there were no ratings at the extreme (agitation) end of the SAS. This is difficult to achieve without purposefully selecting these patients and timing becomes a crucial factor. A significantly longer time frame for data collection would be required. No paired ratings were performed during the night duty hours. Whilst there would be an expectation that patients' sedation scores would be assessed throughout the 24hour period, it is not possible for one researcher to be available for that whole time. Night duty may have an impact on the raters and this requires further consideration. Casual resource and agency staff were not eligible to participate in 
this study. These staff are utilised in ICUs when there is a need and therefore require educating in how the SAS is used within the unit. Brandl et al. (2001) did demonstrate, however the SAS was reliable when used by inexperienced nursing staff. Finally this study is not able to inform us about the staff who did not consent to participate in the research. It cannot be determined whether the nonparticipants are different to the study population. All this study can produce is detail of those staff who participated and what their characteristics are. Due to privacy laws in New Zealand little demographic detail of employees is publicly available.

\section{Reflection on the Research Process}

This is the first time the researcher has led a research project. I found it to be a valuable and rewarding experience. It was useful as the researcher, not to be a participant in the study. It enabled me to be more objective about what was occurring and more able to appraise and reflect on the sedation practice in the ICU. I was also aware that I had two roles in the ICU during the study; one as the Clinical Nurse Specialist (CNS) and one as the researcher. I had anticipated that this may have been problematic but it did not appear to be the case. I was very careful not to influence staff that may or may not have wanted to participate in the study. Other researchers in similar roles may have a different experience and may find it preferable to repeat the research in an ICU they are not affiliated with.

The underlying intention of this study was that the SAS would not be used as a basis for clinical decision-making until such time as its reliability had been determined. Despite this as staff became more familiar with the tool, I noted on a number of occasions staff using a SAS score in discussing with medical staff the sedation needs of individual patients. Nurses had adopted it to help them describe the behaviour of patients in advocating for an alteration in sedation management. I also heard medical staff asking specifically for the SAS score of a patient to enable them to use this in deciding how agitated a patient was and the appropriate sedation management. As the study progressed a number of staff had started to base their decisions for titrating sedatives on the SAS scores. The 
degree of adoption and whether staff had used it as part of their clinical decisionmaking in their practice is something the researcher did not anticipate or have any control over. Its partial adoption however, provides an indication of its perceived use as a helpful clinical tool.

During the research process there were no issues that came about with doing the ratings. Because of the random selection process of patients, the tight time frame for data collection and the exclusion of patients that had already been evaluated; did mean it was difficult to achieve my specific goal of three ratings per day. Although Wellington ICU has over 1200 admissions per year there were some days where there were no new patients to the ICU and ratings could not be performed. There were times when critically ill patients remained in the unit for several days to weeks; this made access to new patients for ratings difficult especially as patients were only evaluated once during their ICU stay. The researcher would recommend in replicating this study that the time frame for data collection be considerably longer. I believe that the patient make-up of the unit, that is, a significant number of surgical admissions enabled me to achieve this objective due to the consistent 'turnover' of patients through the unit. If the makeup of other ICUs is different (that is more medical admissions) a longer time frame may be required to gather an adequate sample.

The ratings procedure itself went as planned. Staff did not appear to attempt to communicate with each other during the procedure. The difficulty lay with getting the primary nurse and doctor together at the bed-space at the time that a set of observations was due to be performed, without other demands impacting. For example, a staff members' meal break, the doctor being off the ICU because they were reviewing another patient or were in the unit but unable to attend because they were busy with the needs of other patients. Instances that occurred that delayed ratings also included the arrival of post-operative cardiac surgical patients, resuscitation situations, and instability of patients which meant time could not be spared for ratings. The researcher and research in a natural setting needs to be flexible and patient. 
The Wellington Regional Ethics Committee, C\&CDHB Maori Health Unit and the staff of the ICU require feedback from this research project. After examination and lodgement of this thesis in the university library, a report will be prepared for the ethics committee and the Maori Health Unit. A summary of the research will also be distributed to the ICU staff. One full copy of the thesis will be given to the ICU and further copies made available for interested persons. Particular staff who requested a copy of the results, as part of their consent, will also receive their own copies. Results will also be presented to the staff in the form of presentations and conferences.

\section{Conclusion}

A model sedation scale should portray accurately the degree of sedation or agitation within well-defined categories, be simple to use, guide the titration of therapy, and have validity and reliability in ICU patients (Jacobi et al., 2002). The SAS is one of the few sedation-scoring scales in existence which has demonstrated through research that these goals are achievable. This research has further confirmed the SAS as a reliable tool for use in general ICUs. Nurses and doctors using the SAS do provide consistent scores and have a mutual understanding of the SAS and its constituent levels.

As a result of this study there are several recommendations that needed to be considered for clinical practice and further research. Firstly there is a need to replicate or develop a similar study; a larger sample population may enable further analysis of variables in considering the association between variables and differences in agreement. This may further enhance the consistency of the SAS. I would also recommend that further research be performed over a longer time frame to ensure sufficient cell size to see if there are any other variables contributing to the difference between raters. Further research with the SAS is required to establish its responsiveness or the degree to which the tool can identify significant changes in sedation over time. Additional appraisal and definition of the language used in the SAS score is required including the removal of the word 'minimum' and the use of the terms 'awaken' and 'arouse'. It 
is imperative that a sedation-scoring tool is introduced with an education programme, guidelines and training for all staff in the ICU and that this is regularly re-visited to ensure staff remain informed on a tool's application. Ultimately an appropriate goal for sedation should be determined each day for individual patients and reassessed on a regular basis as the patients' clinical condition changes. Frequent assessment of the degree of sedation or agitation may facilitate the titration of sedatives to predetermined endpoints.

Consequently there are also several recommendations for clinical practice in the Wellington ICU. Firstly the unit now needs to develop a set of sedation guidelines to support the SAS and support the staff in consistently titrating sedatives to the needs of patients. Supplementary training with the SAS is required on an intermittent basis to ensure staff remain familiar with the tool. Further auditing of the types and modes of sedation used in Wellington ICU is required to ensure there is an appropriate use of the sedatives for individual patients and specific patient groups. In considering this point, there is further deliberation on splitting the morphine and midazolam continuous infusions into separate infusions to allow them to be individually titrated for the specific needs of a patient. Finally, further research at Wellington ICU is required in a post-implementation analysis that measures the impact of the SAS on relevant patient outcomes such as cost of sedation, ventilation hours, length of stay, the need for further diagnostic investigations and the need for tracheostomies. 


\section{Chapter 8- Conclusion}

The management of sedation in the Intensive Care Unit (ICU) is a fundamental, yet complex issue. A significant number of patients in ICU require sedation for at least part of their stay. Sedation relieves anxiety, discomfort and aids treatment. There is a developing body of knowledge around the adverse effects of pain and anxiety; studies have previously established that among ICU patients there are memories of painful experiences and these experiences have been associated with the development of post-traumatic stress disorder (Fontes Pinto Novaes et al., 1999; Rotondi et al., 1998). Despite this, nursing and medical professionals can overlook pain and sedation management. Adequate sedation may become obscured among the myriad of haemodynamic, respiratory and metabolic derangements evident within critically ill patients. The safe and effective application of sedation must be an integral part of intensive care practice and without this critically ill patients are exposed to stress. Although stress is usually considered a compensatory mechanism, within the context of critical care it can produce serious and significant complications, such as hypoxia, tachycardia, increased myocardial oxygen consumption, immunosuppression and persistent catabolism. For these very reasons appropriate sedation is essential to aid the recovery of ICU patients.

Critically ill patients are a heterogeneous group with different underlying comorbid conditions, severity of illness, monitoring needs and requirements for life support. This makes managing sedation in this group very complex. If sedation is considered a continuum, at one end the patient is deeply sedated and unresponsive whilst at the other end they may be awake and co-operative or agitated and distressed. Patients' conditions in ICU change rapidly and so too do their sedative requirements. In managing sedation in ICU you are seeking a balance between over-sedation with its associated complications and undersedation along with its associated complications. The difficulty with sedation is the varied and often unpredictable effect of critical illness on drug pharmacodynamics and pharmacokinetics. 
Assessing the adequacy of sedation can be problematic because of its subjective nature. It is a bedside process in which the nurse's participation is essential; he or she will frequently notice changes in the patients' optimal level of sedation because they are present at the bedside continuously. Preferably the sedation goals for a patient would include comfortable, breathing in synchrony with the ventilator; be orientated and able to follow simple commands (such as squeezing a hand) without precipitating unnecessary agitation. Unfortunately as the severity of illness intensifies, it can become more difficult to reconcile the goals of assuring a calm, comfortable patient while preventing the accumulation of sedatives. This presents a challenge to the nursing staff in trying to titrate sedatives to attain these goals and a challenge to the medical staff in determining what is an appropriate sedative agent for an individual patient. The very nature of the ICU environment makes decision-making complex and at times rapid. There are many contextual factors which influence this decisionmaking all of which may impact on how nurses and doctors make the decisions to assess sedation and manage it for individual patients.

Questions continue to surface over what forms an optimal sedation strategy. Part of many sedation strategies in ICUs today is the use of a sedation-scoring tool. The evolution of sedation-scoring tools has endeavoured to allow nursing and medical staff to provide some consistency to their assessments and the ensuing clinical decisions. It also provides a commonality of understanding as to what is meant by a particular score based on observed patient behaviour. Very few sedation-scoring tools, however, have been evaluated and confirmed as valid and reliable tools for use in clinical practice. Valid and reliable sedation scales are necessary to provide nurses and doctors with a consistent method for assessing level of sedation among patients, to enhance dosing sedatives, and to evaluate the effectiveness of various sedatives in the ICU. For the scale to be effective, the target of sedation (that is, the goal of the scale) must be consistent between doctors and nurses caring for the patient. The Sedation-Agitation Scale (SAS) is one such scale which has had reliability and validity testing performed. 
Until this study however, its reliability between nursing and medical staff had not been confirmed.

On the basis of this study the SAS has been confirmed as a reliable tool for assessing sedation in the Wellington ICU. The ICU can now be more confident in using this tool to evaluate sedation in their patients and a base for clinical decision-making. The researcher anticipates that the SAS will improve the quality of sedation management in critically ill patients as it will facilitate communication between nurses and medical staff with regard to the effectiveness of sedation regimes, and assist with the development of optimal sedation and analgesia guidelines for ICU patients. The SAS offers critical care practitioners an objective tool by which to titrate sedation therapy in ICU patients. Further work is now needed evaluating the effectiveness of sedation regimes and the responsiveness of sedation-scoring tools. 


\section{APPENDIX A- Sedation - Agitation Scale (SAS)}

\begin{tabular}{|c|c|c|}
\hline 7 & Dangerous agitation & $\begin{array}{l}\text { Pulling at ET tube, trying to remove catheters, } \\
\text { climbing over bed rail, striking at staff, } \\
\text { thrashing side-to-side }\end{array}$ \\
\hline 6 & Very agitated & $\begin{array}{l}\text { Does not calm, despite frequent verbal } \\
\text { reminding of limits; requires physical restraints, } \\
\text { biting ET tube }\end{array}$ \\
\hline 5 & Agitated & $\begin{array}{l}\text { Anxious or mildly agitated, attempting to sit up, } \\
\text { calms down to verbal instructions }\end{array}$ \\
\hline 4 & Calm and co-operative & Calm, awakens easily, follows commands \\
\hline 3 & Sedated & $\begin{array}{l}\text { Difficult to arouse, awakens to verbal stimuli or } \\
\text { gentle shaking, but drifts off again, follows } \\
\text { simple commands }\end{array}$ \\
\hline 2 & Very sedated & $\begin{array}{l}\text { Arouses to physical stimuli, but does not } \\
\text { communicate or follow commands, may move } \\
\text { spontaneously }\end{array}$ \\
\hline 1 & Unrousable & $\begin{array}{l}\text { Minimal or no response to noxious stimuli, does } \\
\text { not communicate or follow commands }\end{array}$ \\
\hline
\end{tabular}

Source: Riker, R., Picard, J. and Fraser, G. (1999). Prospective Evaluation of the Sedation Agitation Scale for Adult Critically III Patients. Critical Care Medicine 27:1325-9. 


\section{Appendix B- Ethics Committee Approval Letter}

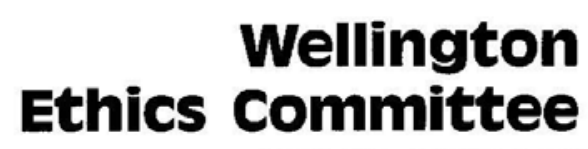

Room 425, Fourth Floor Community \& Support Services Wellington Hospital Private Bag 7902 wellington South

9 July 2003

Phone (04) 3855999 ext. 5185

Fax (04) 3855840

Email: claire.l@wec.org.nz

Wellington Ethics Committee Ref No: 03/06/056

Please quote this reference number in all correspondence relating to this study.

Michelle Ryder-Lewis

$\mathrm{C} /$ - Intensive Care Services

Wellington Hospital

Private Bag 7902

Wellington South

Dear Michelle

03/06/056 - A reliability study of the Sedation - Agitation Scale (SAS) in the Wellington Intensive Care Unit

Thank you for your letter of 2 July 2003 addressing the points raised in my letter to you of 19 June 2003. My apologies for quoting two different reference numbers in my letter of 19 June. In future when referring to this protocol the correct reference number is $03 / 06 / 056$.

As all outstanding ethical issues have now been addressed, the above study has been given ethical approval by the Wellington Ethics Committee.

\section{Accreditation}

This Committee is accredited by the Health Research Council and is constituted and operates in accordance with the Operational Standard for Ethics Committees, March 2002.

\section{Progress Reports}

The study is approved until August 2004. The Committee will review the approved application annually. A progress report is required for this study in July 2004. You will be sent a form requesting this information prior to the review date. Please note that failure to complete and return this form may result in the withdrawal of ethical approval. A final report is also required at the conclusion of the study.

\section{Amendments}

All amendments to the study must be advised to the Committee prior to their implementation, except in the case where immediate implementation is required for reasons of safety. In such cases the Committee must be notified as soon as possible of the change. 


\section{General}

It should be noted that Ethics Committee approval does not imply any resource commitment or administrative facilitation by any health-care provider within whose facility the research is to be carried out. Where applicable, authority for this must be obtained separately from the appropriate manager within the organisation.

We wish you well with your research.

Yours sincerely

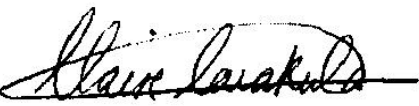

Claire Lavakula

Co-Administrator 


\section{Appendix C- Family Information Sheets}

\section{Victoria \\ UNIVERSITY OF WELLINGTON \\ Te Whare Wãnanga

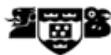

GRADUATE SCHOOL

OF NURSING \& MIDWIFERY

Dear Family and Friends,

I am currently undertaking a research study in the ICU to identify the very best way of recording the sedation of patients (Sedation is medication used to ensure your loved one gets a good sleep or to keep them calm while in ICU). The research involves comparing the way doctors and registered nurses complete an assessment sheet. The registered nurse caring for your loved one will liaise with you about care and planning for your loved one on a day-to-day basis and will keep you informed if ratings are being completed by your loved ones doctor and nurse.

If you would like to know more about the study or have any concerns please do not hesitate to discuss these with me.

Michelle Ryder-Lewis

MA (Applied) Student

Victoria University of Wellington

Graduate School of Nursing and Midwifery and

Clinical Nurse Specialist

Intensive Care Unit

Wellington Hospital, C\&C DHB

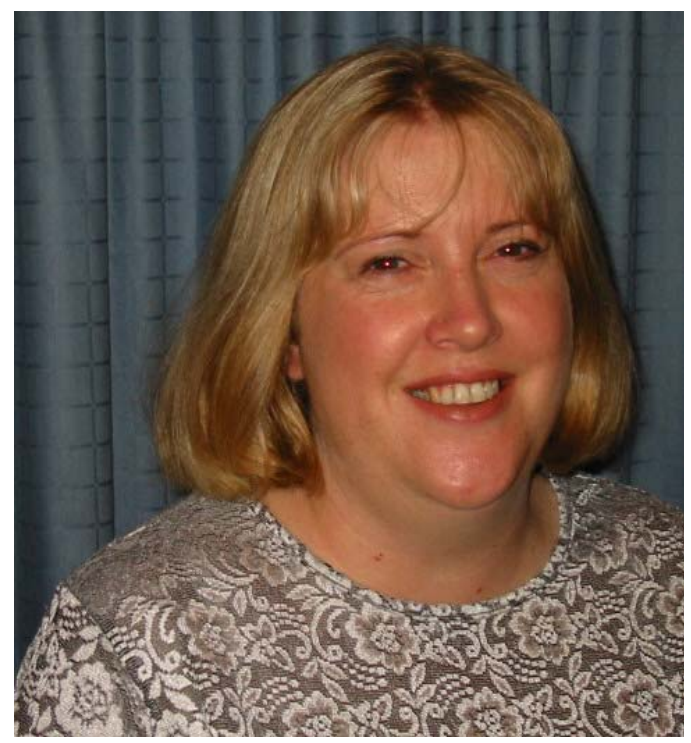

Wellington Ethics Committee Approval No: 03/06/056 


\section{Ethical Approval for the Study}

The Wellington Regional Ethics Committee has approved this study. If you wish to contact them with any concerns, please phone them on 04385 5999, ext 5185. They are based on the Wellington Hospital Campus.

\section{Questions or Queries?}

If you have any questions about the study, please contact me.

I am available in the Intensive Care Unit each weekday.
Michelle Ryder-Lewis

MA (Applied) Student

Graduate School of Nursing \&

Midwifery

Victoria University of Wellington

and

Clinical Nurse Specialist

Intensive Care Services

Wellington Hospital, C\&C DHB

Wellington Ethics Committee Approval No: WGTN 03/06/056

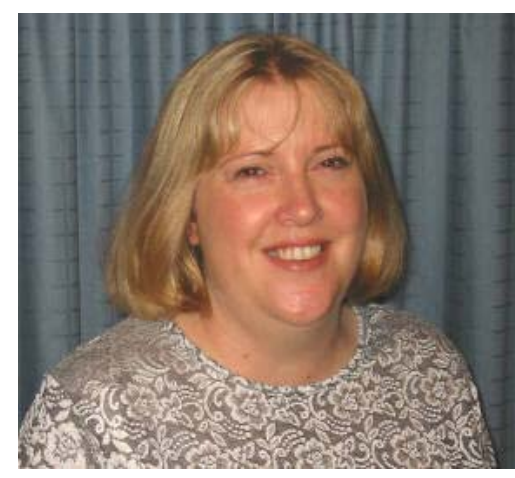

Victoria

UNIVERSITY OF WEL

Te Whare Wānanga

o te Ûpoko o te Ika a Māui

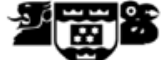

GRADUATE SCHOOL OF NURSING \& MIDWIFERY

\section{A research study to assess the findings of the registered nurse and doctor using a sedation} scoring tool in the Intensive Care Unit 


\section{Dear Family \& Friends}

I am currently working on a research study in the Intensive Care Unit (ICU).

The purpose of the study is to identify the very best way of recording the sedation of patients.

The research involves comparing the way the registered nurses and doctors complete an assessment sheet. Not all the registered nurse's and doctor's in the ICU will be involved in the study. The registered nurse caring for your loved one will liaise with you about care and planning on a day-to-day basis and will keep you informed if ratings are being completed by your loved ones doctor and nurse.

\section{What is sedation?}

Sedation is medication that we use to ensure your loved one gets a good sleep while in ICU. It is also medication that helps to calm the patient.

It is important to us that we provide enough sedation to keep your loved one comfortable and not provide too much or too little of the medication.

\section{What are the participants} going to do?

The nurse and doctor will be observing the way that the patient is managing with their sedation. This takes less than two minutes. The Nurse will call the patient's name, and then will ask the patient to squeeze their hand. Both the nurse and doctor will record their perception of the patients' reaction on a scale from 1-7.

It is important to remember that the participants in the study are the registered nurse and the doctor and it is their perception and judgment that is being measured. Be assured that if your loved one is perceived to be distressed at the time of the assessment staff will always act to ensure the comfort and wellbeing of the patient first and foremost. 


\title{
Appendix D- Participant Information and Consent Form
}

\section{Victoria \\ Te Whare Wānang te Ûpoko o te Ika a Mãui

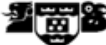 \\ GRADUATE SCHOOL \\ OF NURSING \& MIDWIFER}

\author{
Participant Information Sheet
}

Title:

A reliability study of the Sedation-Agitation Scale (SAS) in the Wellington

Intensive Care Unit

Investigator: Michelle Ryder-Lewis

MA (Applied) Student

Graduate School of Nursing \& Midwifery

Victoria University of Wellington; and

Clinical Nurse Specialist

Wellington Intensive Care Services

Capital \& Coast District Health Board

Phone: 04-385 5999, Ext 6248

Email: Michelle.Ryder_Lewis@ccdhb.org.nz

Supervisors: Kathy Nelson

Lecturer

Graduate School of Nursing \& Midwifery

Victoria University of Wellington

Jane MacGeorge

Lecturer

Graduate School of Nursing \& Midwifery

Victoria University of Wellington; and

Nurse Consultant

Clinical Support Services

Capital \& Coast District Health Board 


\section{Victoria}

UNIVERSITY OF WELLINGTON
Te Whare Wãnanga

o te Üpoko o te Ika a Mãui

\section{중용}

GRADUATE SCHOOL

OF NURSING \& MIDWIFERY

Dear Colleagues

You are invited to take part in this research study, which is a reliability study of the SedationAgitation Scale (SAS) in ICU. Do not feel obliged to make your decision now, feel free to think about it at home and contact myself when you have made your decision. The information provided in this leaflet; should help to explain the study. It is your right to decide not to take part in the study.

\section{About the Study}

The SAS was introduced into practice in Wellington ICU in February 2003. This scale was developed to assist ICU staff to assess patient sedation and/or agitation in determining whether a patient is over/under sedated.

Aim - The proposed research intends to ascertain the reliability of the SAS. Many sedation scoring tools are in use internationally but very few have been tested for validity or reliability. The aim of this study is to determine the reliability of the SAS when used by staff in a tertiary level ICU, in particular whether nurses and doctors do rate patients similarly.

This study is important to Wellington ICU because before the tool can be fully integrated into clinical decision making around patient care, the ICU needs to be confident in its reliability.

What would you be required to do - You are being asked to participate independently in assessing patients in the ICU, using the SAS along with one of your colleagues. The assessment will take less than two minutes. You will be asked to observe some features of the patient's behaviour, such as response to voice, stimulus and ability to obey commands. We will ask you to do this at the time of a routine neurological assessment. Your result will be written on a piece of paper and handed to me. Please be assured that your participation will in no way change the management or interfere with the care of the patients.

If you would like to participate, you are asked to complete some questions about yourself at the end of this consent form. Each of you will then be given a unique Identifying Number. I will be the only person who knows the exact identify of the unique number. These details are needed to describe the characteristics of the ICU staff.

All information collected will be anonymous and remain confidential to the Investigator and her Supervisors. It will not be possible to identify you individually in any reports that are prepared from the study. All raw data will be kept locked in a filing cabinet in a locked office.

I am aiming to recruit permanent staff in the Wellington ICU for the purpose of this study.

If you would like to participate, please complete the attached consent form and return to me in the envelope provided by $31^{\text {st }}$ July 2003 . Your consent form can be left in my mail tray in the ICU. If you wish to discuss the study further, please do not hesitate to contact me in the ICU. 


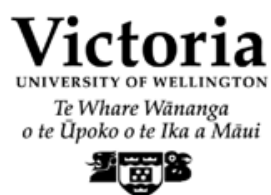

GRADUATE SCHOOL OF NURSING \& MIDWIFERY
2 July 2003

\section{Consent Form}

\section{A reliability study of the Sedation-Agitation Scale (SAS) in the Wellington Intensive Care Unit}

I have read and understand the information sheet dated 2 July 2003 for participants. I have had the opportunity to discuss this study. I am satisfied with the answers I have been given.

I understand that taking part in this study is voluntary and that I may withdraw from the study at any time and the right not respond to any questions.

I understand that Jane MacGeorge, Nurse Consultant, Celia McKiernan, Clinical Nurse Preceptor and Dr Peter Hicks, Clinical Leader of ICU are available, should I not feel comfortable approaching Michelle Ryder-Lewis with any concerns. Support and guidance is available to me through these people.

I understand that my participation in this study is confidential and no material that could identify me will be used in any reports on this study.

I wish to receive a copy of the results. $\quad$ Yes/No

I understand that the Wellington Ethics Committee has approved the research and I may contact them with any concerns. The Wellington Ethics Committee, Wellington Hospital, Telephone 385 5999, Ext 5185.

Statement by Participant: I hereby consent to take part in this study

Name (Print):

Date:

Signature:

Signature

of Researcher:

Date: 
ID No.

RN Level on C\&C DHB CCP

\begin{tabular}{|l|l|}
\hline $2 \mathrm{~A}$ & \\
\hline $2 \mathrm{~B}$ & \\
\hline 3 & \\
\hline 4 & \\
\hline
\end{tabular}

Medical Staff Levels

\begin{tabular}{|l|l|}
\hline Registrar & \\
\hline Consultant & \\
\hline
\end{tabular}

ICU Specialty Course (Circle)

Yes

No

Please Specify:

Number of years worked in any ICU (accumulatively)

Number of years worked in Wellington ICU (accumulatively)

Previous experience with sedation scoring tools (Circle) $\quad$ Yes

Please Specify:

No

Which Ethnic Group(s) do you identify with? (If more than one group, please indicate your order of preference by numbering the boxes.)

NZ European

Mäori

Samoan

Cook Island Mäori

Other:
Tongan

Niuean

Chinese

Indian

please specify 


\title{
Appendix E- Ethics Committee Safety Concerns
}

\author{
Wellington \\ Ethics Committee \\ Room 425, Fourth Floor \\ Community \& Support Services \\ Wellington Hospital \\ Private Bag 7902 \\ Wellington South \\ Phone (04) 3855999 ext. 5185 \\ Fax (04) 3855840 \\ Email: claire.l@wec.org.nz
}

18 June 2003

Wellington Ethics Committee Ref No: 03/06/055

Please quote this reference number in all correspondence relating to this study.

Michelle Ryder-Lewis

$\mathrm{C} /$ - Intensive Care Services

Wellington Hospital

Private Bag 7902

Wellington South

Dear Michelle

03/06/056 - A reliability study of the Sedation - Agitation Scale (SAS) in the Wellington Intensive Care Unit

The above study was considered by the Wellington Ethics Committee at its meeting of 10 June 2003.

The study was approved subject to the following points being addressed:

1 What safeguards are in place to ensure that the patients will not be adversely affected in any way?

2 What measures have been taken to ensure the staff will not feel coerced into participation?

33.5 randomisation is built on the assumption that most staff will give consent. Is this a fair assumption? If they don't, how will this affect the results.

46.3 you need to show awareness of and consider the possibility of psychological risks to staff and address this issue.

5 Letter to family and friends, first paragraph, last sentence, the phrase "if the comparison of forms" is not clear, needs to be better explained. Please change this in the notice also.

6 The information sheet is potentially misleading in the sense that it could be misinterpreted by family members that the audit has implications for health intervention of patients. Please change this to read better in notice also.

When the above points have been addressed, ethical approval for this study will be granted.

Yours sincerely

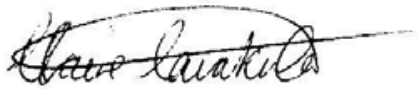

Claire Lavakula

Co-Administrator 


\section{VICTORIA UNIVERSITY OF WELLINGTON \\ Te Whare Wananga o te Upoko o te Ikn a Maui}

2 July 2003

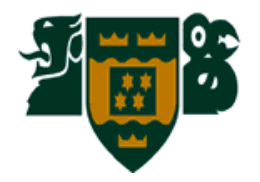

Wellington Ethics Committee

Private Bag 7902

Wellington South

\section{Dear Claire}

Ref No: 03/06/055 A reliability study of the Sedation-Agitation Scale (SAS) in the Wellington Intensive Care Unit.

Thank you for your letter dated 18 June 2003. I appreciate the feedback you have given me with regard to my reliability study of the Sedation-Agitation Scale in the Wellington Intensive Care Unit (ICU). I have given consideration to the points you have made in your letter and have in turn addressed them below.

1. All patients in the ICU will continue to be monitored whilst the ratings are in progress. Should a monitor alarm occur, the rating will be stopped immediately and the alarm addressed. Both the nurse and doctor caring for the individual patient will be at the bedside during the rating therefore a monitor alarm will be addressed immediately. If any patient is found to be distressed at the time of a rating, the rating will not go ahead and staff will act immediately to ensure the comfort and wellbeing of the patient first and foremost. In addition, as referred to in section 17.1 of my application before ratings are done I will ensure that there are no major crises occurring in the ICU. If emergency situations are occurring, or staff are pressured or extremely stressed, I have the experience to make the judgment that these are not appropriate times to be doing data collection.

2. There will be a three-week period for which staff can consider whether to consent to participating in this study, prior to data collection commencing. I have built this in to provide staff time to consider their consent without any coercion from myself. Staff who do not wish to participate will not return a consent form. Any communication with staff regarding the consent forms will be of a general nature and no individuals will be approached during this time period.

3. I believe my assumption with regard to section 3.5 is fair given that I have built the study on the largest possible number of staff doing ratings, the staff in the ICU have indicated to me that they are keen and interested in this study, however if less people do consent to participate there will be no impact on the results as I have determined that 120 ratings are only required to produce significant results and this can be achieved by staff (who have consented) doing more than one rating.

4. I do not believe that there is a risk to the staff in the ICU. Even if two staff members reveal that they rated a patient differently, I do not believe this will have 
an impact as the purpose of this research is to determine whether staff do rate the SAS similarly. However in considering the possibility of psychological risks to staff, I have included a statement in the participant consent form which states "I understand that Jane MacGeorge, Nurse Consultant; Celia McKiernan, Clinical Nurse Preceptor; and Dr. Peter Hicks, Clinical Leader of ICU are available should I not feel comfortable approaching Michelle Ryder-Lewis with any concerns. Support and guidance is available to me through these people". The consent form also states that staff may withdraw consent at any time they wish to. I have enclosed an updated version of the consent form for your information. Staff at Capital \& Coast District Health Board also have free access to an Employee Assistance Program, which offers counselling and support with any issue. Staff within the ICU do access this program and all staff are provided with information on how to access this when they commence employment in the ICU. The information is also displayed on the staff notice board in the ICU. Support and guidance is available to me through these people and my supervisor, Kathy Nelson.

5. Thank you for your comments about the family and friends letter. I have given thought to your comments and have made changes to the wording of the letter. I have attached a copy for your information.

6. I have also made the corresponding changes to the family information pamphlet. Your feedback on this issue has been valuable.

I trust that these issues have addressed the concerns that the committee has raised. Thank you for your assistance in this research proposal. I will look forward to hearing from you shortly.

For your information, I have noted on your letter that you have given me two different reference numbers. Please could you confirm for me which is the correct one for me to use with all correspondence.

Yours Sincerely

Michelle Ryder-Lewis.

Student, Graduate School of Nursing and Midwifery

C/o Intensive Care Service

Wellington Hospital

Private Bag 7902

Wellington South. 


\section{Appendix F-Sedation Score Data Sheet}

SEDATION Score DAta SHEET

TO BE COMPLETED BY LEAD INVESTIGATOR

Date $(\mathrm{dd} / \mathrm{mm})$ :

Time of Rating (e.g. 0800 hrs):

Patient's Details:

Age (in years):

Gender:

Male

Female

Ethnicity (Classified on Hospital Admission Sheet):

$\square$ NZ European

Mäori

Samoan

$\square$ Cook Island Mäori

$\square$ other

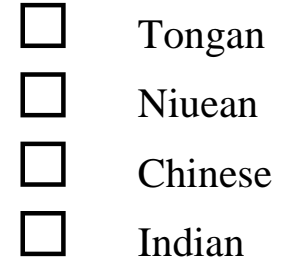

Please specify

Length of Stay in ICU (in days):

APACHE II Score:

APACHE II Diagnosis Code:

Intubated Yes

No

Any Neuromuscular blockade given in last 30 minutes: $\quad$ Yes $\quad \square \quad$ No

Type of Sedation Bolus $\square \quad$ Infusion

Nurse Unique Identifier:

Nurse SAS Score:

Doctor Unique Identifier:

Doctor SAS Score:

For Office Use Only

Entered

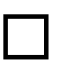

Checked

$\square$ 


\section{Appendix G- APACHE II Diagnostic Codes}

\begin{tabular}{|c|c|c|c|c|c|}
\hline \multirow[b]{2}{*}{ Code } & \multirow[b]{2}{*}{ Cardiovascular Nonoperative } & \multicolumn{2}{|c|}{ APACHE 2 Diagnostic Codes } & \multirow[b]{2}{*}{305} & \multirow[b]{2}{*}{ Hysterectomy } \\
\hline & & Code & Metabolic/renal Nonoperative & & \\
\hline 109 & Hypertension & 122 & Drug overdose & Code & Respiratory Nonoperative \\
\hline 110 & Congestive heart failure & 123 & Diabetic keto-acidosis & 101 & Asthma \\
\hline 112 & Acute myocardial infarction & 305 & Renal disease & 102 & COPD \\
\hline 114 & Cardiac arrest & 306 & Other Haematologic disease & 103 & Pulmonary oedema (non cardiac) \\
\hline 115 & Cardiogenic shock & 306 & Coagulopathy/Neutro/Thrombop & 104 & Aspiration pneumonia \\
\hline 116 & Aortic aneurysm & 307 & Other metabolic disease & 105 & Pulmonary embolism \\
\hline 117 & Rhythm disturbance & 307 & Metabolic coma & 106 & Bacterial/viral Pneumonia \\
\hline 302 & Peripheral vascular disease & Code & Metabolic/renal Operative & 106 & Parasitic pneumonia \\
\hline 302 & Other Cardiovascular disease & 215 & Renal neoplasm & 107 & Resp neoplasm (inc larynx/trachea) \\
\hline Code & Cardiovascular Operative & 216 & Renal transplant & 108 & Respiratory arrest \\
\hline 202 & Carotid endarterectomy & 305 & Surgery for other renal disease & 303 & Other Respiratory disease \\
\hline 202 & AAA & Code & Neurologic Nonoperative & 303 & Mechanical airway obstruction \\
\hline 202 & Peripheral arterial bypass graft & 120 & Suharachnoid haemerchno & Code & Respiratory Operative \\
\hline 202 & PVD - no graft & 121 & Subarachnoid haemorrhage & 209 & Respiratory neoplasm - lung \\
\hline 202 & Dissecting/ruptured aorta & 121 & Intracerebral haemorrhage & 211 & Respiratory insufficiency after surgery \\
\hline 203 & Valvular heart surgery & 301 & Stroke & 303 & Respiratory infection \\
\hline 302 & Other Cardiovascular disease & 301 & Neurologic infection & 303 & Other Respiratory disease \\
\hline 308 & CABG & 301 & Neurologic neoplasm & 303 & $\begin{array}{l}\text { Respiratory neoplasm - } \\
\text { mouth/larynx/sinc:s/trachea }\end{array}$ \\
\hline Code & Gastro-intestinal Nonoperative & 301 & Other Neuroiogic disease & & \\
\hline $\begin{array}{l}124 \\
124\end{array}$ & $\begin{array}{l}\text { Gl bleeding - diverticulosis } \\
\text { Gl bleeding - varices }\end{array}$ & $\begin{array}{l}\text { Code } \\
217\end{array}$ & $\begin{array}{l}\text { Neurologic Operative } \\
\text { Subarachnoid haemorrhage }\end{array}$ & 113 & Sepsis (urinary tract origin) \\
\hline 304 & Other GI disease & 217 & Intracerebral haemorrhage & 113 & Sepsis with shock (non-urinary) \\
\hline 304 & GI inflammatory disease & & Subdural/epidural haematoma & 113 & Sepsis with shock (urinary) \\
\hline & (UC/Crohns/Pancreatitis) & 218 & Craniotomy for neoplasm & 118 & Multiple trauma (excluding head) \\
\hline 304 & Hepatic failure & 219 & Laminectomy/spinal cord surgery & 118 & Multi-trauma + spinal cord injury \\
\hline 304 & Gl perforation/obstruction & 301 & Surgery for other neurologic disease & 118 & Isolated cervical cord inury \\
\hline $\begin{array}{l}\text { Code } \\
212\end{array}$ & Gastro-intestinal Operative & Code & Other Nonoperative & 119 & Head trauma $+1-$ multi-trauma \\
\hline 212 & Gl bleeding & 111 & CVS failure from Haem & Code & Sepsis/trauma Operative \\
\hline 213 & GI neoplasm & & shock/nypovolaemıa & 204 & Sepsis (any aetiology after surgery) \\
\hline 214 & Gi perforation/rupture & 308 & $\begin{array}{l}\text { Other med disease (nil above } \\
\text { appropriate) }\end{array}$ & 207 & Multiple trauma (excluding head) \\
\hline 214 & GI obstruction & Code & Other Operative & 207 & Multitrauma + spinal injury \\
\hline 304 & Gl inflammatory Disease & 205 & Haemorrhagic shock (after surgery) & 207 & Burns \\
\hline 304 & Cholecystitis/cholangitis & 206 & $\begin{array}{l}\text { Post cardiac arrest (after or during } \\
\text { surgery) }\end{array}$ & 208 & Head trauma +1 - multi-trauma \\
\hline 304 & Liver transplant & 207 & Hip or extremity fracture & & \\
\hline 304 & Other GI disease & 305 & Pregnancy related surgery & & \\
\hline
\end{tabular}




\section{Appendix H- Letters Confirming Permission to Reprint}

From: "William Trochim" <wmt1@cornell.edu>

To: "Michelle Ryder-Lewis" <michelleRN@xtra.co.nz>

Subject: Re: Fw: Reproduction of diagrams

Date: Sunday, 21 September 2003 5:17 p.m.

You have my permission to use the diagrams. Good luck with your thesis.

At 10:43 AM 9/21/2003 +1200, you wrote:

>"urn:schemas-microsoft-com:office:office">

$>$

$>-----$ Original Message -----

$>$ From: <mailto:michelleRN@xtra.co.nz>Michelle Ryder-Lewis

>To: <mailto:wmt1@cornell.edu>wmt1@cornell.edu

$>$ Sent: Sunday, July 20, 2003 7:10 PM

$>$ Subject: Reproduction of diagrams

$>$

$>$ Dear Sir,

$>$

>My name is Michelle Ryder-Lewis, I am the Clinical Nurse Specialist of the

$>$ Intensive Care Unit (ICU) at Wellington Hospital, New Zealand. I am

>currently undertaking a research project as part of my Masters degree in

$>$ Nursing at Victoria University of Wellington. My research is a reliability

>study of the Sedation-Agitation Scale in Wellington ICU. I am required to

>complete a thesis as part of this Masters degree. In my thesis I am

$>$ writing a chapter on true score theory and reliability. I wish to use some

$>$ diagrams from your website in order to illustrate my explanations of these

$>$ complex concepts. The diagrams I would like to reproduce in my thesis are

> under the chapter on reliability are; true score theory, measurement

>error, random error, inter-rater reliability, test-retest reliability and

>parallel forms reliability. In any future publications relating to my

$>$ research, these diagrams would not be included. This is only to support

$>$ the discussion in my thesis. I will of course ensure it is referenced

$>$ correctly using the guidelines-publication manual of the American

$>$ Psychological Association. I am seeking your permission to reproduce these

$>$ diagrams as part of my discussion of the background to my research thesis

$>$ methodology. If there is any other information you require please do not

$>$ hesitate to contact me. I also want to thankyou and congratulate you on

$>$ your website. It was such a relief to me to find information of such

>complex issue well laid out and easy to understand. Your information has

>guided me in the development of my research proposal. I look forward to

$>$ your reply in the near future.

$>$

$>$ Yours Sincerely

$>$

$>$ Michelle Ryder-Lewis

$>$

$>$ MA (Applied) Student

$>$

$>$ Graduate School of Nursing \& Midwifery 
>Victoria University of Wellington; and

$>$

$>$ Clinical Nurse Specialist

$>$

$>$ Wellington Intensive Care Services

$>$

$>$ Capital \& Coast District Health Board

$>$

>Phone: 04-385 5999, Ext 6248

>Email: <mailto:michelleRN@xtra.co.nz>michelleRN@xtra.co.nz

$>$

$>$

$>$

$>$

William M. Trochim

Professor

Policy Analysis \& Management

249 MVR Hall

Cornell University

Ithaca, NY 14853

607-255-0887

607-255-4071 fax

http://trochim.cornell.edu/ 


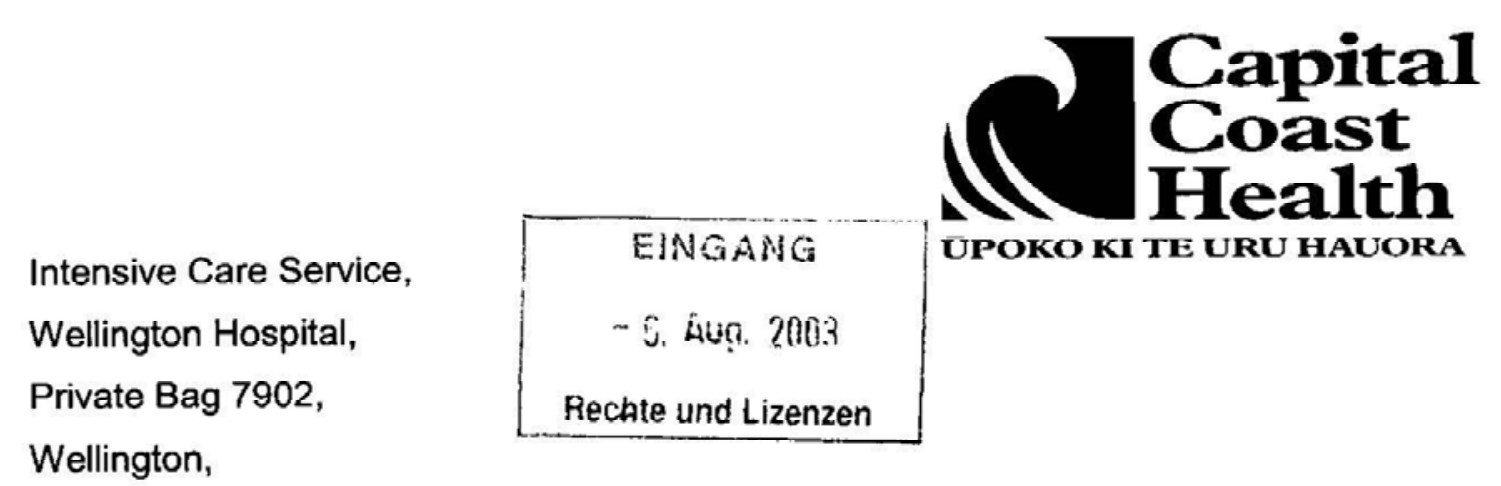

New Zealand.

21-07-2003.

Intensive Care Medicine,

Springer- Verlag, Ulrike Butz,

Journal Production Department,

Heidelberger Platz 3,

14197 Berlin,

Germany.

Dear Sir, Madam.

My name is Michelle Ryder-Lewis, I am the Clinical Nurse Specialist of the Intensive Care Unit (ICU) at Wellington Hospital, New Zealand. I am currently undertaking a research project as part of my Masters degree at Victoria University of Wellington. My research is a reliability study of the SedationAgitation Scale in Wellington ICU. I am required to complete a thesis as part of this Masters degree. In my thesis I wish to use a table from an article published in your journal. The article is;

De Jonghe, B._Cook, D., Appere-De-Vecchi, C., Guyatt, G, Meade, M. \& Outin, $H$. (2000). Using and understanding sedation scoring systems: A systematic review. Intensive Care Medicine, 26(3): 275-285.

In this article an extensive table (Table2) summarises the scales used for sedation in critically ill patients which underwent formal methodological evaluation. I wish to reproduce part of this table in my thesis as part of my literature review discussion on sedation-scoring tools and reliability testing. I 
would like to use the following parts of this table; the Ramsay scale, the Sedation-Agitation Scale and the Motor Activity Assessment Scale. I will of course ensure it is referenced correctly using the guidelines-publication manual of the American Psychological Association. I am seeking your permission to reproduce this part of Table 2 as part of my discussion of the literature in my thesis. If there is any other information you require please do not hesitate to contact me. I look forward to your reply in the near future.

Yours Sincerely

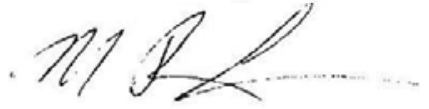

Michelle Ryder-Lewis

MA (Applied) Student

Graduate School of Nursing \& Midwifery

Victoria University of Wellington; and

Clinical Nurse Specialist

Wellington Intensive Care Services

Capital \& Coast District Health Board

Phone: 04-385 5999, Ext 6248

Email: Michelle.Ryder_Lewis@ccdhb.org.nz

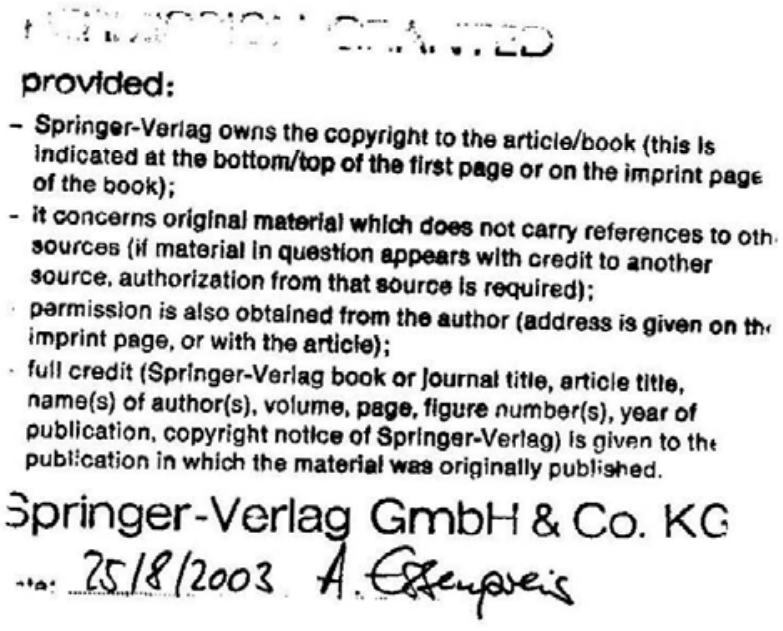




\section{References}

Alexander, C.S., Weisman, C.S., \& Chase, G.A. (1982). Determinants of staff nurses' perceptions of autonomy within different clinical contexts. Nursing Research, 31 (1), 48-52.

Allen, D. (1997). The nursing medicine boundary: negotiated order. Sociology of Health and IIIness, 19(4), 498-520.

Altman, D.G. (1991). Practical statistics for medical research. London: Chapman and Hall.

Analyse-it@ (2003). Analyse-it@-the most popular statistical software add-in for Microsoft Excel (for windows). Retrieved 25 $5^{\text {th }}$ November,2003, from http://www.analyse-it.com/start.htm.

Anderson, J., \& O'Brien, M. (1995). Challenges for the future: the nurses role in weaning patients from mechanical ventilation. Intensive and Critical Care Nursing, $11(1), 2-5$.

Audit Commission (1999). Critical to success: The place of efficient and effective critical care services within the acute hospital. London: Audit Commission.

Baker, J.D. (1997). Phenomenography: an alternative approach to researching the clinical decision-making of nurses. Nursing Inquiry, 4, 41-47.

Bakken Henry, S. (1991). Effect of level of patient acuity on in clinical decisionmaking of critical care nurses with varying levels of knowledge and experience. Heart and Lung, 20 (5), 478-485.

Benner, P. (1984). From novice to expert. Excellence and power in clinical nursing practice. San Francisco: Addison-Wesley Publishing Co. 
Benner, P., Hooper-Kyriakidis, P., \& Stannard, D. (1999). Clinical wisdom and interventions in critical care. A thinking-in-action approach. Philadelphia: W.B. Saunders Co.

Benner, P. \& Tanner, C. (1987). Clinical judgment: How expert nurses use intuition. American Journal of Nursing, January, 23-31.

Benner, P., \& Wrubel, J. (1989). The Primacy of Caring. Stress and Coping in Health and IIIness. California: Addison- Wesley Publishing Co.

Botha, J.A., \& LeBlanc, V. (2002, October). The state of sedation in the nation: Results of an Australian survey. Paper presented at $27^{\text {th }}$ Australian and New Zealand Annual Scientific Meeting on Intensive Care, Perth, Western Australia.

Bowler, S., \& Mallik, M. (1998). Role extension or expansion: a qualitative investigation of the perceptions of senior medical and nursing staff in an adult intensive care unit. Intensive and Critical Care Nursing, 14, 11-20.

Bradshaw, A. (1998). Charting some challenges in the art and science of nursing. The Lancet, 351 (100), 438-443.

Brandl, K.M., Langley, K.M., Riker, R.R., Dork, L.A., Qualls, C.R., \& Levy, H. (2001). Confirming the reliability of the Sedation-Agitation Scale administered by ICU nurses without experience in its use. Pharmacotherapy, 21(4), 431-436.

Brink, P., \& Wood, M. (1998). Advanced design in nursing research $\left(2^{\text {nd }}\right.$ ed.). California: Sage Publications.

Brook, A.D., Ahrens, T.S., Schaiff, R., Prentice, D., Sherman, G., Shannon, W., \& Kollef, M.H. (1999). Effect of a nursing-implemented sedation protocol on the duration of mechanical ventilation. Critical Care Medicine, 27 (12), 2609-2615.

Bucknall, T. (2000). Critical care nurses decision-making activities in the natural clinical setting. Journal of Clinical Nursing, 9, 25-36. 
Bucknall, T. (2003). The clinical landscape of critical care: nurses' decisionmaking. Journal of Advanced Nursing, 43(3), 310-319.

Bucknall, T., \& Thomas, S. (1997). Nurses' reflections on problems associated with decision-making in critical care settings. Journal of Advanced Nursing, 25, 229-237.

Buresh, B., \& Gordon, S. (2000). From silence to voice. What nurses know and must communicate to the public. Ontario: Canadian Nurses Association.

Burns, N., \& Grove, S. (2001). The practice of nursing research conduct, critique and utilisation ( $4^{\text {th }}$ ed.). Philadelphia : W.B. Saunders Co.

Capital Coast Health. (1998). Nursing and midwifery career pathway information and guidelines. Wellington: Capital Coast Health.

Carper, B. (1978). Fundamental patterns of knowing in nursing. Advances in Nursing Science, 1, 13-23.

Clark, A. (1998). The qualitative-quantitative debate: Moving from positivism and confrontation to post-positivism and reconciliation. Journal of Advanced Nursing, 27, 1242-1249.

Cook, D.J., Walter, S.D., Cook, R.J., Griffith, L.E., Guyatt, G.H., Leasa, D., Jaeschke, R.Z., et al. (1998). Incidence of and risk factors for ventilatorassociated pneumonia in critically ill patients. Annuals of Internal Medicine, 129, 433-440.

Cook, T., \& Campbell, D. (1979). Quasi-experimentation: Design and analysis issues for field settings. Chicago: Rand McNally College Publishing Co.

Cook, S., \& Palma, O. (1989). Propofol as a sole agent for prolonged infusion in intensive care. Journal of Drug Development, 4(suppl 2), 65-67. 
Coombs, M. (2003). Power and conflict in intensive care clinical decision making. Intensive and Critical Care Nursing, 19, 125-135.

Dean, B. (1998). Reflections on technology: Increasing the science but diminishing the art of nursing? Accident and Emergency Nursing, 6, 200-206.

De Jong, M.J., \& Karch, A.M. (2000). Critical care drug guide. Philadelphia: Lippincott Williams \& Wilkins.

De Jonghe, B., Cook, D., Appere-De-Vecchi, C., Guyatt, G., Meade, M., \& Outin, H. (2000). Using and understanding sedation scoring systems: A systematic review. Intensive Care Medicine, 26(3): 275-285.

De Jonghe, B., Cook, D., Sharshar, T., Lefaucheur, J., Carlet, J., \& Outin, H. (1998). Acquired neuromuscular disorders in critically ill patients: A systematic review. Intensive Care Medicine, 24, 1242-1250.

Department of Health. (2001). The nursing contribution to the provision of comprehensive critical care for adults: A strategic program of action. London: Author.

Devlin, J.W., Boleski, G., Mlynarek, M., Nerenz, D., Peterson, E., Jankowski, M., et al. (1999). Motor Activity Assessment Scale: A valid and reliable sedation scale for use with mechanically ventilated patients in an adult surgical intensive care unit. Critical Care Medicine, 27, 1271-1275.

Devlin, J.W., Fraser, G.L., Kanji, S., \& Riker, R.R. (2001). Sedation assessment in critically ill adults. The Annals of Pharmacotherapy, 35, 1624-1632.

Egerod, I. (2002). Uncertain terms of sedation in ICU. How nurses and physicians mange and describe sedation for mechanically ventilated patients. Journal of Clinical Nursing, 11, 831-840.

Epstein, J., \& Breslow, J.M. (1999). The stress response of critical illness. Critical Care Clinics, 15, 17-33. 
Field, D. (1989). Nursing the dying. London: Tavistock-Routledge.

Fontes Pinto Novaes, M.A., Knobel, E., Bork, A.M. (1999). Stressors in ICU: Perception of the patient, relatives and health care team. Intensive Care Medicine, 25, 1421-1426.

Fraser, G.L., \& Riker, R.R. (2001). Monitoring sedation, agitation, analgesia, and delirium in critically ill adult patients. Critical Care Clinics, 17(4), 967-987.

Gerdtz, M.F., \& Bucknall, T.K. (1999). Why we do the things we do: applying clinical decision-making frameworks to triage practice. Accident and Emergency Nursing, 7, 50-57.

Gilbertson, A.A. (1995). Before intensive therapy? Journal of the Royal Society of Medicine, 88, 459p-463.

Gillis, A., \& Jackson, W. (2002). Research for nurses. Methods and interpretation. Philadelphia : F.A. Davis Co.

Gunn, D. (1999). Linking nurse prescribing and advanced practice. Kai Tiaki Nursing New Zealand, June, 25.

Guyatt, G., Walter, S., \& Norman, G. (1987). Measuring change over time: Assessing the usefulness of evaluative instruments. Journal of Chronic Disease, 40, 171-178.

Hogg, K. (1994). Don't let cure be at the expense of care. Professional Nurse, 9 , 465-470.

Jacobi, J., Fraser, G.L., Coursin, D.B., Riker, R.R., Fontaine, D., Wittbrodt, E.T., et al. (2002). Clinical practice guidelines for the sustained use of sedatives and analgesics in the critically ill adult. Critical Care Medicine, 30(1), 119-141.

Jenner, C. (1997). The art of nursing: A concept analysis. Nursing Forum, 32 (4), 5-7. 
Johnson, J. (1996). Dialectical analysis concerning the rational aspect of the art of nursing. Image: Journal of Nursing Scholarship, 28 (2), 169-175.

Karmel, L., \& Karmel, M. (1978). Measurement and evaluation in the schools $\left(2^{\text {nd }}\right.$ ed.). New York : MacMillan Publishing Co.

Koch, K.A., Rodeffer, H.D., \& Wears, R.L. (1994). Changing patterns of terminal care management in an intensive care unit. Critical Care Medicine, 22, 233-243.

Kollef, M., Levy, N., Ahrens, T., Schaiff, R., Prentice, D., \& Sherman, G. (1998). The use of continuous IV sedation is associated with prolongation of mechanical ventilation. Chest, 114(2), 541-548.

Knaus, W.A., Wagner, D.P., Draper, E.A., Zimmerman, J.E., Bergner, M., Bastos, P.G., et al. (1991). The APACHE II prognostic system. Risk prediction of hospital mortality for critically ill hospitalized adults. Chest, 100(6), 1619-1636.

Kress, J.P., Pohlman, A.S, \& Hall, J.B. (2002). Sedation and analgesia in the intensive care unit. American Journal of Critical Care Medicine, 166, 1024-1028.

Kress, J., Pohlman, A., O'Connor, M., \& Hall, J. (2000). Daily interruption of sedative infusions in critically ill patients undergoing mechanical ventilation. New England Journal of Medicine, 342, 1471-1477.

Lawler, J. (1991). Behind the screens: Nursing, somology, and the problem of the body. London: Churchill-Livingstone.

Lerch, C., \& Park, G.R. (1999). Sedation and analgesia. British Medical Bulletin. 55(1), 76-95.

LoBiondo-Wood, G., \& Haber, J. (1994). Nursing research methods, critical appraisal and utilisation ( $3^{\text {rd }}$ ed.). St. Louis : Mosby. 
Lynaugh, J.E., \& Fairman, J. (1992). New nurses, new spaces: a preview of the AACN history study. American Journal of Critical Care, 1, 19-24.

Lynn, M. (1989). Reliability estimates: Use and disuse. In F. Downs (Ed.), Readings in research methodology ( ${ }^{\text {nd }}$ ed.) (p.236-238). Philadelphia: Lippincott Williams \& Wilkins.

Maas, M., \& Buckwalker, K. (1989). Quasi-experimental designs. In Brink, P., \& Wood, M. (Ed.), Advanced Design in Nursing Research (p.57-87). California: Sage Publications.

Magarey, J. (1997). Sedation of adult critically ill ventilated patients in intensive care units: a national survey. Australian Critical Care, 10(3), 90-93.

Manias, E. (1998). Possibilities for collaboration: a critical ethnography of nursenurse and nurse-doctor relationships in intensive care. Unpublished Doctor of Philosophy Thesis. La Trobe University, Australia.

Manias, E., \& Street, A. (2000). Legitimation of nurses' knowledge through policies and protocols in clinical practice. Journal Advanced Nursing,32 (6), 14671475.

Manias, E., \& Street, A. (2001a). Nurse-doctor interactions during critical care ward rounds. Journal of Clinical Nursing,10, 442-450.

Manias, E., \& Street, A. (2001b). Nurses and doctors communicating through medication order charts in critical care. Australian Critical Care, 14(1), 17-23.

McConnell, E. (1998). The coalescence of technology and humanism in nursing practice: It doesn't just happen and it doesn't come easily. Holistic Nursing Practice, July, 23-31.

Meade, M.O., Cook, D.J., Kernerman, P., \& Bernard, G. (1997). How to use articles about harm: The relationship between high tidal volumes, ventilating 
pressures, and ventilator-induced lung injury. Critical Care Medicine, 25, 19151922.

Medicines Act. (1981). New Zealand Government: Wellington.

McGaffigan, P. (2002). Advancing sedation assessment to promote patient comfort. Critical Care Nurse, February, 29-37.

MIMS (2003). Medsafe datasheet, Fentanyl. Retrieved October 2, 2003, from the MIMS New Zealand Database.

Ministry of Health (2002). Guidelines for the development and operation of standing orders. Wellington: Author.

Mowinski Jennings, B., \& Rogers, S. (1989). Managing measurement error. In F. Downs (Ed.), Readings in research methodology (2 $2^{\text {nd }}$ ed.) (p.239-241). Philadelphia: Lippincott Williams \& Wilkins.

Nightingale, F. (1863). Notes on hospitals. Longmans, Green.

Nursing Council of New Zealand. (2002a). Medicines (Designated Prescriber: Nurses Practicing in Aged Care) Notice 2002. New Zealand Gazette, 163, 4105.

Nursing Council of New Zealand. (2002b). Medicines (Designated Prescriber Practicing in Child Family Health) Notice 2002. New Zealand Gazette,163, 4106.

Nursing Council of New Zealand. (2002c). The Nurse Practitioner ${ }^{\mathrm{TM}}$. Responding to health in New Zealand ( $3^{\text {rd }}$ ed.). Wellington: Author.

Olson, D.M., Chioffi, S.M., Macy, G.E., Meek, L.G., \& Cook, H.A. (2003). Potential benefits of Bispectral Index monitoring in critical care. A case study. Critical Care Nurse, 23 (4), 45-52. 
Ostermann, M., Keenan, S., Seiferling, R., \& Sibbald, W. (2000). Sedation in the intensive care unit, a systematic review. Journal of the American Medical Association, 283(11), 1451-1459.

Peat, J. (2002). Health science research. A handbook of quantitative methods. London: Sage Publications.

Poole, K., \& Jones, A. (1996). A re-examination of the experimental design for nursing research. Journal of Advanced Nursing, 24, 108-114.

Prescriptions come naturally for first prescriber. (2003, July). New Zealand Nursing Review, 4 (3), 3.

Ramsay, M., Savege, T., Simpson, B., \& Goodwin, R. (1974). Controlled sedation with Alphaxalone-Alphadolone. British Medical Journal, ii, 656-659.

Ray, M. (1987). Technological caring: A new model in critical care. Dimensions of Critical Care Nurse, 6 (1), 166-173.

Reiser, S.J. (1992). The intensive care unit. The unfolding and ambiguities of survival therapy. International Journal of Technology Assessment in Health Care, 8, 382-394.

Ridley, S., Biggman, M., \& Stone, P. (1990). A cost-benefit analysis of intensive therapy. Anaesthesia, 48, 14-19.

Riker, R.R., Fraser, G.L., \& Cox, P. (1994). Continuous infusion of haloperidol controls agitation in critically ill patients undergoing haemodiafiltration. Critical Care Medicine, 22, 433-440.

Riker, R.R., Fraser, G.L., \& Wilkins, J. (2000). Bilateral versus unilateral EEG monitoring with Bispectral Index: A comparison among adult ICU patients [abstract]. Critical Care Medicine, 28(12 suppl), 56. 
Riker, R.R., Picard, J., \& Fraser, G.L. (1999). Prospective evaluation of the Sedation Agitation Scale for adult critically ill patients. Critical Care Medicine, 27, 1325-1329.

Riker, R.R., Simmons, L.E., Fraser, G. L., \& Wilkins, J. (2001). Validating the Sedation-Agitation Scale with the bispectral index and visual analog scale in adult ICU patients after cardiac surgery. Intensive Care Medicine, 27, 853-858.

Robins, L.N. (1995). How to choose among the riches: Selecting a diagnostic instrument. International Journal of Methods in Psychiatric Research, 5, 103-109.

Rogers, E.M. (2003). Diffusion of innovations (5 ${ }^{\text {th }}$ ed.). New York: The Free Press.

Rotondi, A., Schulz, R., \& Sirio, C. (1998). The experiences of patients receiving prolonged mechanical ventilation in the ICU. Chest, 114, $328 \mathrm{~S}$.

Rowley, G., \& Fielding, K. (1991). Reliability and accuracy of the Glasgow coma scale with experienced and inexperienced users. Lancet, 337, 535-538.

Royal College of Nursing Critical Care Forum. (1997). The nature of nursing work in intensive care results of a telephone survey. London: Royal College of Nursing.

Sakr, M., Angus, J., Perrin, J., Nixon, C., Nicholl, J., \& Wardrope, J. (1999). Care of minor injuries by emergency nurse practitioners or junior doctors: $A$ randomized controlled trial. The Lancet, 354, 1321-1326.

Salvage, J. (1990). The theory and practice of the 'new nursing'. Nursing Times Occasional Paper, 86, 42-45.

Sandelowski, M. (1998). Looking to care or caring to look? Technology and the rise of spectacular nursing. Holistic Nursing Practice, July, 1-11.

Sessler, C.N., Gosnell, M.S., Grap, M.J., Brophy, G.M., O’Neal, P.V., Keane, K.A., Tesoro, E.P. et al. (2002). The Richmond Agitation-Sedation Scale. Validity 
and reliability in adult intensive care unit patients. American Journal of Respiratory Critical Care Medicine,166, p.1338-1344.

Seymour, J.E. (2001). Critical moments-death and dying in intensive care. Buckingham: Open University Press.

Shehabi, Y., \& Innes, R. (2002). Sedation and analgesia in the $21^{\text {st }}$ century. English Journal of Anaesthesia, 18, 143-155.

Simmons, L., Riker, R.R., Prato, B., \& Fraser, G.L. (1999). Assessing sedation during intensive care unit mechanical ventilation with the Bispectral Index and the Sedation-Agitation Scale. Critical Care Medicine, 27(8), 1499-1504.

Skodol Wilson, H. (1993). Introducing Research in Nursing, (2 ${ }^{\text {nd }}$ ed.). California: Addison-Wesley Nursing.

Slavitt, D.B., Stamps, P.L., Peidmont, E.B., \& Haase, A.M. (1978). Nurses satisfaction with their work situation. Nursing Research, 27, 114-120.

Slutsky, M.P. (1994). Consensus conference on mechanical ventilation. Part 1. Intensive Care Medicine, 20, 64-79.

Smythe, E. (1987). The poet as our mentor. Nursing Praxis in New Zealand, 2 (2), 3-7.

Soliman, H.M., Mèlot, C. \& Vincent, J-L. (2001). Sedative and analgesic practice in the intensive care unit: the results of a European survey. British Journal of Anaesthesia, 87(2), 186-192.

Stein, L.I., Watts, D.T., \& Howell, T. (1990). The doctor nurse game revisited. Nursing Outlook, November/December, 264-268.

Stern, H.P., Stroh, S.E., Fiser, D.H., Cromwell, E.L., McCarthy, S.G., \& Prince, M.T. (1991). Communication, decision-making, and perception of nursing roles in a paediatric intensive care unit. Critical Care Nurses Quarterly,14 (3), 56-68. 
Streiner, D.L., \& Norman, G.R. (1995). Health management scales. A practical guide to their development and use $\left(2^{\text {nd }} \mathrm{ed}\right)$. Oxford: Oxford University Press.

Svensson, R. (1996). The interplay between doctors and nurses: a negotiated order perspective. Sociology of Health and IIIness, 18, 379-398.

Tobin, M.J. \& Fahey, P.J. (1994). Management of the patient who is 'fighting the ventilator'. In M.J. Tobin (Ed), Principles and Practices of Mechanical Ventilation (p.1149-1162). New York: McGraw-Hill.

Trochim, W. (2002). The research methods knowledge base, $\left(2^{\text {nd }}\right.$ ed.). Retrieved $24^{\text {th }}$ September, 2002, from http://trochim.human.cornell.edu/kb/contents.htm.

Trubuhovich, R.V., \& Judson, J.A. (2001). Intensive care in New Zealand. A history of the New Zealand region of ANZICS. Auckland: Department of Critical Care Medicine, Auckland Hospital.

Tu, J.V., Jaglal, S.B., \& Naylor, C.D. (1995). Multicenter validation of a risk index for mortality, intensive care unit stay, and overall hospital length of stay after cardiac surgery. Circulation, 91, 677-684.

Wajcman, J. (1991). Feminism Confronts Technology. Cambridge: Polity Press.

Walby, S., \& Greenwell, J. (1994). Medicine and nursing: Professions in a changing health service. London: Sage Publications.

Watts, T., Jones, M., Wainwright, P., \& Williams, A. (2001). Methodologies analysing individual practice in health care: A systematic review. Journal of Advanced Nursing, 35 (2), 238-256.

Weightman, W.M., Gibbs, N.M., Sheminant, M.R., Thackray, N.M., \& Newman, M.A.J. (1997). Risk prediction in coronary artery surgery: a comparison of four risk scores. Medical Journal of Australia, 166, 408-411. 
Weinert, C.R., Chlan, L., \& Gross, C. (2001). Sedating critically ill patients: factors affecting nurses' delivery of sedative therapy. American Journal of Critical Care, 10(3), 156-165.

Welch, M. (1999). Phenomenology and hermeneutics. In Polifroni, C. \& Welch, M. (Ed.). Perspectives on Philosophy of Science in Nursing, (p. 235-246). Philadelphia: Lippincott.

Whale, Z. (1993). The participation of hospital nurses in the multidisciplinary ward round on a cancer-therapy ward. Journal of Clinical Nursing,2, 155-163.

White, S., Hollet, J., Kress, J., \& Zellinger, M. (2001). A renaissance in critical care nursing: Technological advances and sedation strategies. Critical Care Nurse, 21(5), 1-14.

Willatts, S.M. (1990). The application of scoring systems in adult intensive care. Baillière's Clinical Anaesthesiology, 4(2), 253-269. 